\title{
Dynamical and stereodynamical studies of alkaline-earth atom-molecule reactions
}

\author{
KING-CHUEN LIN† and A. GONZÁLEZ UREÑA* \\ $\dagger$ Department of Chemistry, National Taiwan University, Taipei, and Institute of Atomic \\ and Molecular Sciences, Taipei, Taiwan 106, Republic of China \\ †Unidad de Láseres y Haces Moleculares, Instituto Pluridisciplinar, Universidad \\ Complutense de Madrid. Juan XXIII, 1. 28040 - Madrid, Spain
}

(Received 29 June 2006; in final form 10 January 2007)

\begin{abstract}
Alkaline-earth atom molecule reactions have been extensively investigated since the early days of reaction dynamics. Though these reactions have many similarities to those of corresponding alkali atoms, as one would expect from their low ionization potential, there are important differences due to the presence of the two valence electrons in the alkaline-earth atom. This feature confers a distinct dynamical or stereodynamical behaviour whose basic description and analysis is the main objective of this article. The adopted approach is phenomenological based on the huge body of data revealed by modern crossed-beam and laser pump and probe techniques with no intention to be comprehensive but rather to put into context both authors' work. The paper starts with a resumé of the experimental approaches followed by a visit to full-collision studies under gas cell, beam-gas and crossed-beam arrangements. Detailed analysis and discussion is given of the $\mathrm{M}+\mathrm{H}_{2}(\mathrm{M}=\mathrm{Ca}, \mathrm{Mg}) \rightarrow \mathrm{MH}+\mathrm{H}$ reaction family whose dynamics is discussed using their Potential Energy Surfaces. In these studies care was taken to discuss the most relevant features of the molecular reaction dynamics with emphasis on the energy selectivity and on the stereodynamical (vectorial) nature of the reaction. To this end the available data are discussed in light of topological features of the underlying potential energy surface favouring either insertion or abstraction mechanisms. Particular attention is paid to the discussion of alkaline-earth atom reactions with halogen containing molecules. In these reactions the presence of two valence electrons leads to reaction channels, as chemiluminescent or chemi-ionization reactions, which are specifically associated with the transfer of the more energetic (inner harpooning) electron, whose dynamics or stereodynamics pattern differs from those of reaction channels associated to the transfer of the first, less energetic electron as, for example, in ion-pair reactions. A full section of the paper is dedicated to photo-induced intracluster reactions and, in particular, to the transition state dynamics of the prototype $\mathrm{Ba} \cdot \cdots \mathrm{FCH}_{3}+h v \rightarrow \mathrm{BaF}+\mathrm{CH}_{3}$ reaction investigated in great detail by both nanosecond and femtosecond photo-ion and photo-electron pump and probe techniques. For this system the combination of these ultrahigh resolution techniques with isotopic labelling and $a b$ initio calculations allowed the deciphering of its ultrafast reaction mechanism. Finally, the paper ends with some concluding remarks based on the observed propensity rules or general trends found in these alkaline-earth atom reactions.
\end{abstract}

*Corresponding author. Email: laseres@pluri.ucm.es 
Contents PAGE

$\begin{array}{lr}\text { 1. Introduction } & 290\end{array}$

2. Experimental approaches: set-up and methodologies 293

3. Full-collision studies 299

3.1. Gas cell studies by the pump-probe technique 299

$\begin{array}{lll}3.1 .1 \mathrm{Mg}+\mathrm{H}_{2} & 300\end{array}$

$\begin{array}{lll}3.1 .2 \mathrm{Ca}+\mathrm{H}_{2} & 305\end{array}$

$\begin{array}{ll}3.1 .3 \mathrm{Mg}+\mathrm{CH}_{4} & 306\end{array}$

$\begin{array}{lll}3.1 .4 \mathrm{Ca}+\mathrm{CH}_{4} & 308\end{array}$

3.2. Beam-gas studies: total reaction cross-section determined from
beam-gas experiments

3.2.1 Product reaction distributions by probing the nascent vibro-rotational distribution by laser spectroscopy $\quad 312$

$\begin{array}{ll}\text { 3.2.2 Product polarization and stereodynamics } & 317\end{array}$

3.3. Crossed-beam studies: translational energy effects 319

$\begin{array}{ll}\text { 3.3.1 Dynamical and stereodynamical effects in excited reactions } & 327\end{array}$

3.3.2 Product energy disposal and electronic branching ratio 334

4. Half collisions: photo-induced intracluster reactions 335

4.1. Photo-induced intracluster reactions by nanosecond pump and probe techniques: the intracluster $\mathrm{Ba} \cdots \mathrm{FCH}_{3}+\mathrm{h} v \rightarrow$ product reaction

4.2. Photo-induced intracluster reactions by femtosecond pump and probe studies: the $\mathrm{Ba} \cdots \mathrm{FCH}_{3}+h v \rightarrow$ product reactions

5. Concluding remarks

Acknowledgements

References

\section{Introduction}

In the early days of reaction dynamics, reactions of alkali atoms plus halogencontaining compounds were widely studied by the crossed-beam method [1]. These reactions are known to occur via the electron jump mechanism. Here the important step involves an electron transfer from the alkali atom to the reactant molecule, normally to an antibonding orbital of this molecule, which subsequently dissociates under the electrostatic influence of the alkali ions.

Later on this interest was extended to the study of alkaline-earth metals and halogen compounds. Several reasons motivated this interest such as, for example, the possibility of using optical detection techniques on alkaline-earth monohalides, which was not feasible for alkali halides. Pioneering work in this field was that of Zare and coworkers with the study of $\mathrm{Ba}+\mathrm{XH} \rightarrow \mathrm{BaX}(\mathrm{X}=\mathrm{Cl}, \mathrm{F}, \mathrm{Cl}, \mathrm{I})+\mathrm{H}$ reactions by laser-induced fluorescence of the nascent $\mathrm{BaX}[2]$. 
The reactions of heavier alkaline-earth atoms with halogen-containing molecules have many similarities to those of corresponding alkali atoms. This similar behaviour is based on their low ionization potentials as well as in the dissociative attachment. Nevertheless, the presence of two valence electrons in alkaline-earth atoms may originate some differences in the reaction dynamics if the reaction involves divalent oxygen atoms or molecules containing two halogen atoms. An example is the prototype $\mathrm{M}(\mathrm{M}=\mathrm{Mg}, \mathrm{Ca}, \mathrm{Sr}$, and $\mathrm{Ba})+\mathrm{X}_{2}(\mathrm{X}=\mathrm{F}, \mathrm{Cl}, \mathrm{Br}$, and $\mathrm{I})$ reaction family leading to the following product channels:

(a) $\mathrm{M}^{+}+\mathrm{X}_{2}^{-}$ion-pair formation;

(b) $\mathrm{MX}^{*}+\mathrm{X}$ excited, chemiluminescent reaction;

(c) $\mathrm{MX}+\mathrm{X}$ ground state reaction;

(d) $\mathrm{MX}^{+}+\mathrm{X}^{-}$chemionization reaction.

Some of the reaction channels outlined above necessarily imply the transfer of a second electron. For example channel (d) is directly associated with the second electron transfer mechanism. Furthermore, the ion pair formation, channel (a), is generally associated with the transfer of the less energetic electron (outer harpooning), whereas the chemiluminescent reaction, channel (b), involves the transfer of the more energetic (inner harpooning) electron.

In this review article recent examples of alkaline-earth atom reactions with halogencontaining examples will be discussed in light of both harpooning models, i.e. outer and inner harpooning mechanisms with emphasis on the specific dynamical features involved in each type of electron transfer.

Another consequence of the fact that alkaline-earth atoms have an $n s^{2}$ valence-shell electronic structure is the formation of species of the type $\mathrm{H}-\mathrm{M}-\mathrm{X}$, which result from insertion of the metal atom into the hydrogen halide bond. They are stable and could be participating in a complex-mediated reaction mechanism. Thus, the divalent character of alkaline-earth atoms manifests itself in the presence of a deep well in the potential energy surface of these reactions. Two possibilities appear for the transformation from reactants to products in the alkaline-earth atoms with hydrogen halide molecules, $\mathrm{M}$ $(\mathrm{M}=\mathrm{Be}, \mathrm{Mg}, \mathrm{Ca}, \mathrm{Sr}$, and $\mathrm{Ba})+\mathrm{HX}(\mathrm{X}=\mathrm{F}, \mathrm{Cl}, \mathrm{Br}$, and $\mathrm{I}) \rightarrow \mathrm{MX}+\mathrm{H}$, a direct halogen abstraction pathway, which involves a harpoon mechanism, and an insertion pathway, in which the $\mathrm{H}-\mathrm{M}-\mathrm{X}$ potential well is sampled. In this review, examples of each category will be studied and discussed.

The $\mathrm{M}+\mathrm{HX}$ reaction family also shows a particular mass combination, namely, that of 'heavy atom + heavy-light diatomic molecule'. The significant difference between reactant and product reduced masses largely dictates the fate of the reactant angular momentum resulting in efficient channelling of the reactant orbital angular momentum into product rotation. Indeed, as the mass of the metal and halogen atoms increases, the kinematical constraint becomes more pronounced. A good example is the $\mathrm{Ba} \lessgtr \mathrm{HI}$ system discussed in this article.

For the last decade or so major advances in the reaction dynamics of alkaline-earth atoms with molecular hydrogen and its isotopes have become possible because of the availability of $a b$ initio potential energy surfaces which has made it possible to unravel the detailed reaction pathways of excited reaction dynamics, e.g. $\mathrm{Ca}^{*}+\mathrm{H}_{2}(v=0)$ and $\mathrm{Ca}^{*}+\mathrm{H}_{2}(v=1)$. 
The preparation of an electronically excited alkaline-earth atom also opens up interesting possibilities when a linearly polarized laser is used. It makes possible the orbital alignment of the excited atoms with respect to the relative velocity vector that is well defined in a crossed-beam experiment.

Pioneering work in this field was carried out by Rettner and Zare [3] who reported significant polarization effects on product state branching and cross-section in the reaction of $\mathrm{Ca}\left({ }^{1} \mathrm{P}_{1}\right)$ with $\mathrm{HCl}$ and $\mathrm{Cl}_{2}$. To fully understand the alignment effect, the relation between the asymptotically prepared orbital alignment and the collision complex geometry must be established.

For small impact parameters, parallel, $\sigma$ alignment, in the centre-of-mass (c.m.) system transforms to parallel body-fixed $\Sigma$ alignment. However, at larger impact parameters, the $\sigma$ alignment may give rise to parallel $\Sigma$ or perpendicular $\Pi$ alignment depending upon the degree of adiabaticity. The non-adiabatic behaviour implies a nonadiabatic transition at which point $\Omega$ the projection of the electronic orbital angular momentum on the body-fixed axis changes, i.e. $|\Delta \Omega|=1$. Adiabatic behaviour implies $\Delta \Omega=0$, i.e. the conservation of $\Omega$ as the reagents approach.

A well-known model that incorporates this adiabatic behaviour is the so-called 'orbital following' model which has been tested in many atom-atom collisions. The occurrence of 'orbital following', also referred to as orbital locking phenomena, depends on the magnitude of the splitting between the $\Sigma$ and $\Pi$ energy curves. In other words, it depends on whether $\Omega$ is a good quantum number or not. In general, orbital locking takes place when the $\Omega$ splitting is large relative to the angular velocity of the collision.

In this review article, recent examples of stereodynamics of the alkaline-earth atoms plus halogen-containing compounds will be discussed, in particular, some examples that elucidate the role of the electronic orbital alignment in light of the orbital locking picture.

Cluster reactions, and particularly laser intracluster reactions, have become a vigorous and growing field. One reason for such a development has been the application of laser vaporization methods to produce supersonic beams of clusters and van der Waals (vdW) complexes of virtually any element of the periodic table. As a result, cluster beams containing alkaline-earth atoms have been studied using both nanosecond and femtosecond pump and probe techniques. In this review, the spectroscopy and induced chemical reaction of $\mathrm{vdW}$ molecules containing $\mathrm{Ca}, \mathrm{Ba}$, and other alkaline-earth atoms will be discussed.

Examples of the link between transition state spectroscopy and the underlying intracluster reaction will be reviewed, connecting frequency and time-domain aspects of the photo-fragmentation dynamics for alkaline-earth atom clusters.

This review article is not comprehensive. Rather, it summarizes both authors' work, putting their contributions into the more general context of reaction dynamics of alkaline-earth atoms. The paper has, therefore, been organized as follows.

After this introduction a short section describes the main experimental approaches used to investigate reaction dynamics of the alkaline-earth atoms. Then two main sections constitute the core of the article. Section 3 deals with full collision studies covering gas cell, beam-gas, and beam-beam studies. Emphasis is put on new developments and findings, both in the dynamics and stereodynamics of these 
alkaline-earth atom reactions. Section 4 reviews the cluster photo-dissociation or laserinduced intracluster reactions using both nanosecond and femtosecond pump and probe studies. The emphasis is on transition state dynamics from both the frequency and time domain perspectives. The article closes with comments about future directions.

\section{Experimental approaches: set-up and methodologies}

In addition to crossed-beam experiments, the pump-probe technique employed in bulk gas is a feasible alternative for investigating alkaline-earth reactions. In these experiments, a heat-pipe oven or quartz cell at several hundred ${ }^{\circ} \mathrm{C}$ provides $\sim$ mtorr of alkaline earth atoms, together with collision partners at several torr (to ensure pseudo-first-order conditions). The endoergic reaction is initiated by a pump laser pulse that promotes population to an excited state of the alkaline-earth atom. In this manner, dependence of the reaction on energetics, polarization, and states selected may be carried out. The resultant products are monitored through the use of a probe laser pulse that is delayed several nanoseconds with respect to the first pulse, so that relaxation has no time to occur at these pressures. The experiment is well adapted to study the influence of potential energy and electronic symmetry over reactivity, because the electronic excitation can be easily prepared in various quantum states of the atom, including fine structure. In the bulk, velocity distributions are typically MaxwellBoltzmann, such that the determination of angular and kinetic distributions becomes difficult. However, this type of experiment provides an effective way to gain insight into product rovibrational distributions and subsequent energy disposal [4, 5].

Breckenridge and Umemoto pioneered the use of the pump-probe technique to study the $\mathrm{Mg}\left(3 \mathrm{p}^{1} \mathrm{P}\right)+\mathrm{H}_{2} \rightarrow \mathrm{MgH}+\mathrm{H}$ reaction. They observed that the rotational state distribution of $\mathrm{MgH}$ is bimodal and concluded that the reaction proceeds through two microscopic pathways [6-8]. Many other applications follow, while dealing with alkali and alkaline-earth-hydrogen reactions [8-14]. Some refinements of the technique were also developed in well-adapted reactions to better control a few parameters of the reactive collision [15-20].

An example of the pump-probe technique employed in the reaction of $\mathrm{Ca}\left(3 \mathrm{~d}^{1} \mathrm{D}_{2}\right)$ with $\mathrm{H}_{2}$ is shown in figure 1 [21]. A $915 \mathrm{~nm}$ pump laser was used to prepare the $\mathrm{Ca}\left(3^{1} \mathrm{D}_{2}\right)$ via two-photon absorption. After $30 \mathrm{~ns}$ a counterpropagating probe pulse (line width $<0.1 \mathrm{~cm}^{-1}$ ) excited laser-induced fluorescence (LIF) of $\mathrm{CaH}$ via the $\mathrm{B}^{2} \Sigma^{+} \leftarrow \mathrm{X}^{2} \Sigma^{+}$transition. The pump and probe beams $(1.1 \mathrm{~mJ}$ and $<100 \mu \mathrm{J}$, respectively) overlapped in the centre of a six-armed heat-pipe oven, where $\mathrm{Ca}$ was heated to $890 \mathrm{~K}$, yielding a vapour pressure $\sim 20$ mtorr. The $\mathrm{H}_{2}$ gas at $\sim 1.2$ torr flowed slowly through the chamber. LIF from $\mathrm{CaH}$ passed through a bandpass filter and was detected by a photo-multiplier tube enclosed in a cooler at $-20^{\circ} \mathrm{C}$. Laser-induced fluorescence (LIF) spectra of nascent $\mathrm{CaH}(v=0$ and 1$)$ are shown in figure 2 . The $(0,0)$ and $(1,1)$ bands were excited, while the $(0,1)$ and $(1,2)$ emission bands were monitored. Rotational lines were selected for the linear dependence measurements of $\mathrm{H}_{2}$ pressure and pump-probe delay time, to ensure that the obtained rotational distributions are free from interference of rotational cooling and secondary reaction processes. 


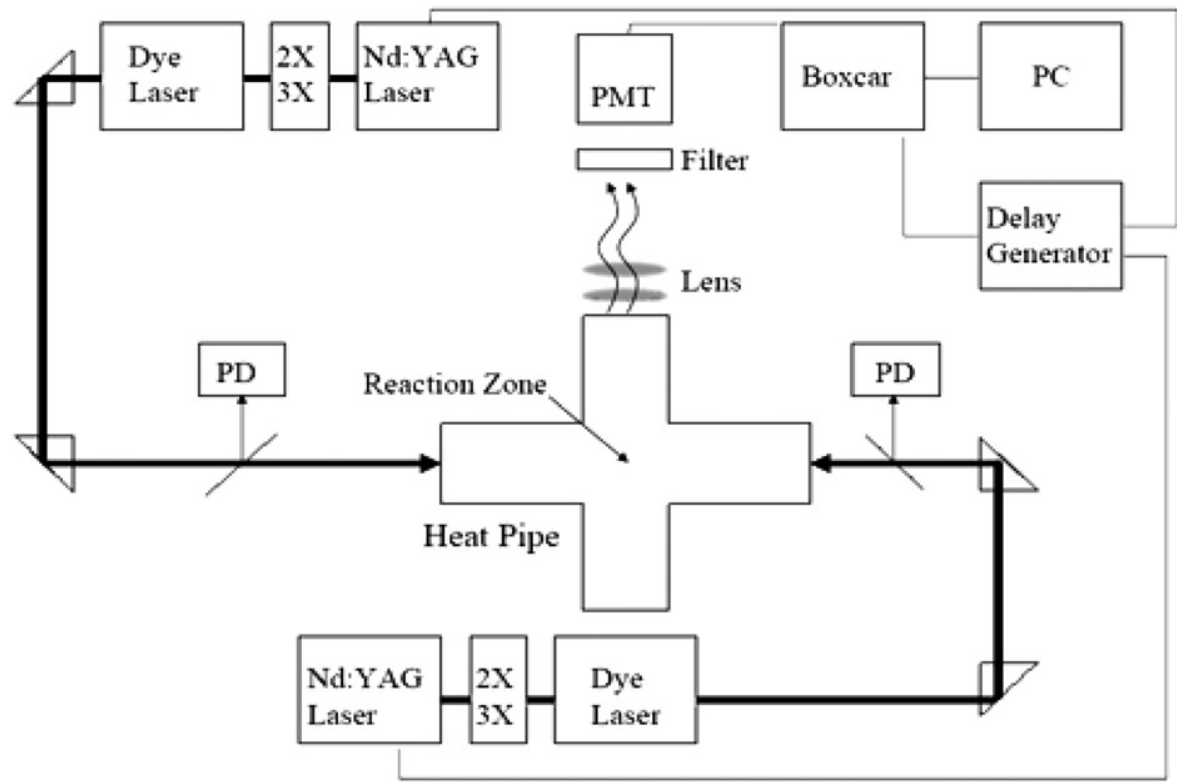

Figure 1. Schematic diagram of a pump-probe technique applied to the $\mathrm{Ca}+\mathrm{H}_{2}$ reaction carried out in a heat-pipe oven. This figure is reproduced with permission from [21].

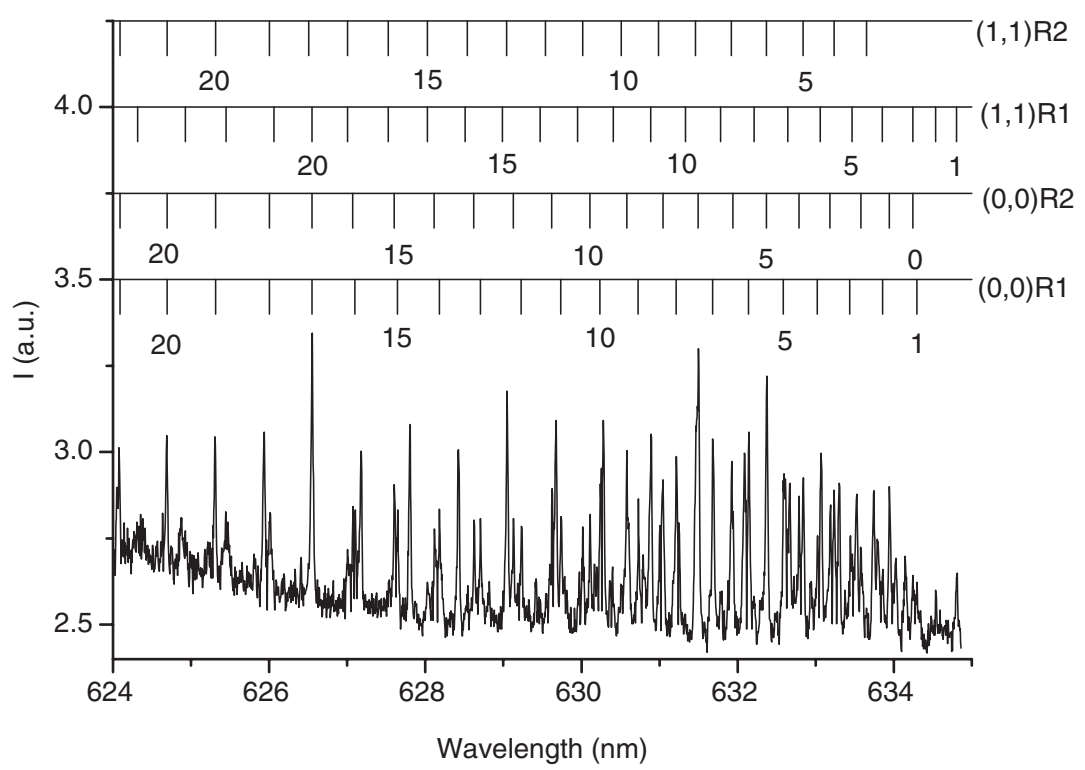

Figure 2. Laser-induced fluorescence (LIF) spectra of $\mathrm{CaH}(v=0$ and 1$)$ in the $\mathrm{B}^{2} \Sigma^{+}-\mathrm{X}^{2} \Sigma^{+}$transition. The $(0,0)$ and $(1,1)$ bands are excited, while the $(0,1)$ and $(1,2)$ emission bands are monitored. This figure is reproduced with permission from [21]. 
If the pump-probe experiments are combined with the stimulated Raman pumping technique, energy can be deposited in the vibrational levels of the collision partner (say $\mathrm{H}_{2}$ ) to inspect the vibrational excitation influence on the reaction. The fraction of population in the vibrational excitation can be estimated by using either Coherent antiStokes Raman spectroscopy (CARS) [22-24] or resonance-enhanced multiphoton ionization (REMPI) [25]. Following photolysis of a metal-containing molecule, it is possible to acquire polarization-dependent Doppler-resolved LIF spectra of the products when the resulting fast metal atoms react with a collision partner in a gas chamber. Vectorial information can be extracted through a complicated algorithmic analysis, similar to the reactions of $\mathrm{H}+\mathrm{N}_{2} \mathrm{O}, \mathrm{CO}_{2}$, etc. [26, 27]. Nevertheless, unlike the $\mathrm{H}$ atom, generation of a well-defined high-speed metal atom is not trivial. On the whole, there are some restrictions to the use of pump-probe technique in the bulk:

(i) the metal atoms in the ground state may not be reactive toward the collision partners;

(ii) metals with higher melting point than alkaline-earth elements may not be suitable to use in a heat-pipe oven, because it usually resists temperature up to $1000^{\circ} \mathrm{C}$.

As is well known, supersonic expansions facilitate the formation of weakly bound van der Waals (vdW) molecules [28]. Early molecular beam studies were limited to gasphase species where, by the use of co-expansion with an inert gas, cooling could be enhanced, allowing the formation of vdW clusters. This technique was used in the early 1970s to study the reactivity of $\left(\mathrm{CH}_{3} \mathrm{I}\right)_{\mathrm{n}}$ with alkali atoms [29].

In addition to the seeding technique there are other methods to produce clusters such as the pick-up technique. The latter is an efficient technique to deposit guest atoms or molecules on large host clusters. It was developed by Scoles and coworkers [30] and is now widely used. In its original version, a secondary beam, containing the guest particle, crossed the supersonic expansion beam forming the clusters just in front of its skimmer. Collisions between both the host clusters and the guest particles led to the guest particles being trapped by the clusters.

More recently, the pick-up technique in the form of a beam-gas arrangement has been developed to produce clusters. An example is $\mathrm{HI} \cdot \cdots \mathrm{Xe}_{\mathrm{n}}$ clusters. The xenon clusters were produced by supersonic expansion of pure xenon; subsequently, the cluster beam passed a pick-up cell containing $\mathrm{HI}$ at $\sim 10^{-2}$ mbar. This type of beam-gas cluster modification has also been adopted to deposit metal atoms and halogen-containing reactants on large clusters to study so-called cluster-isolated chemical reactions (see below).

A well-developed method for producing supersonic molecular beams of highly refractory metals uses a pulsed laser to ablate material from a solid target into a channel attached to a pulsed high-pressure valve [31]. In the supersonic expansion formed on leaving the channel, a variety of metal atoms, vdW clusters, and molecules can be produced, allowing for their study in beam experiments.

Once a well-characterized beam of van der Waals molecules is produced, experiments such as photo-depletion, photo-dissociation and photo-ionization can be undertaken, yielding information about the electronic structure and photo-fragmentation dynamics of these molecules. Essentially, four methods have been developed to investigate 
chemical reactions, which are photo-induced near a transition state region. For these four modalities the reaction is either:

(i) induced by electronic excitation in a $1: 1 \mathrm{vdW}$ complex of two reactants (the pre-reactive complex); or

(ii) it is turned on by photo-detachment in a molecular anion; or

(iii) it is photo-induced in reactants ordered at clean surfaces; or finally

(iv) it is photo-induced in a vdW complex that results from the association between reactants pre-deposited at the surface of large argon clusters - the latter method is the so-called Cluster Isolated Chemical Reaction (CICR) method [32].

The photo-induction of a chemical reaction in a van der Waals complex (A $\cdots$ BC) constitutes a well-established procedure to investigate the elementary chemical reaction near the transition state region and, hence, to investigate transition state spectroscopy both in the frequency and the time domain.

This approach is an example of the so-called half-collision formalism. The originality of the methodology now lies in the fact that the excited complex can not only decay via a non-reactive photo-dissociation, i.e. leading to $\mathrm{A}+\mathrm{BC}$, but it can also undergo chemical reaction, producing $\mathrm{AB}+\mathrm{C}$. Since the complex was formed preparing the reagents within a specific range of precursor $\mathrm{A} \cdot \cdots \mathrm{BC}$ geometries, based on a narrow range of impact parameters, this approach has the advantage of reaching reactive configurations within the transition state region with sufficient density to investigate both the transition state (spectroscopy) and its dynamics.

Another advantage of this method is the possibility of carrying out the reaction dynamics studies using frequency or time domain spectroscopy. In the former implementation both photo-depletion and action spectra can be measured, depending upon the type of measurements employed. In the photo-depletion studies, one measures the complex depletion as a function of the excitation laser wavelength. The complex signal is normally measured by laser ionization, before and after the complex has been excited by a (photo-depletion) laser wavelength. Under no-saturation conditions the two complex signals, $N_{0}$ and $N$, i.e. before and after photo-depletion has taken place, can be related by a Beer-Lambert law type equation, namely

$$
\ln \frac{N_{0}}{N}=\sigma_{\mathrm{d}} \cdot F
$$

Here $\sigma_{\mathrm{d}}$ and $F$ represents the depletion cross-section and the laser fluence, respectively. Thus, a typical photo-depletion spectrum consists of measuring the extent of depletion, taken $\Delta N=N_{0}-N$ as a function of the photo-depletion laser wavelength.

A typical apparatus employed in this type of investigations is shown in figure 3.

The weakly bound complex $\mathrm{Ba} \cdot \cdot \cdot \mathrm{FCH}_{3}$ is produced in a laser vaporization source, followed by supersonic expansion. A gas pulse from a mixture of $\mathrm{He}$ with $\mathrm{CH}_{3} \mathrm{~F}$ is generated, and the output of a Nd:YAG laser is focused onto the surface of a rotating barium disk to produce $\mathrm{Ba}$ vapour that is injected into the gas pulse. This mixture expands supersonically into the vacuum chamber.

The molecular beam is then probed inside the acceleration region of a linear timeof-flight mass spectrometer, using the fourth harmonic output of a Nd-YAG laser for 


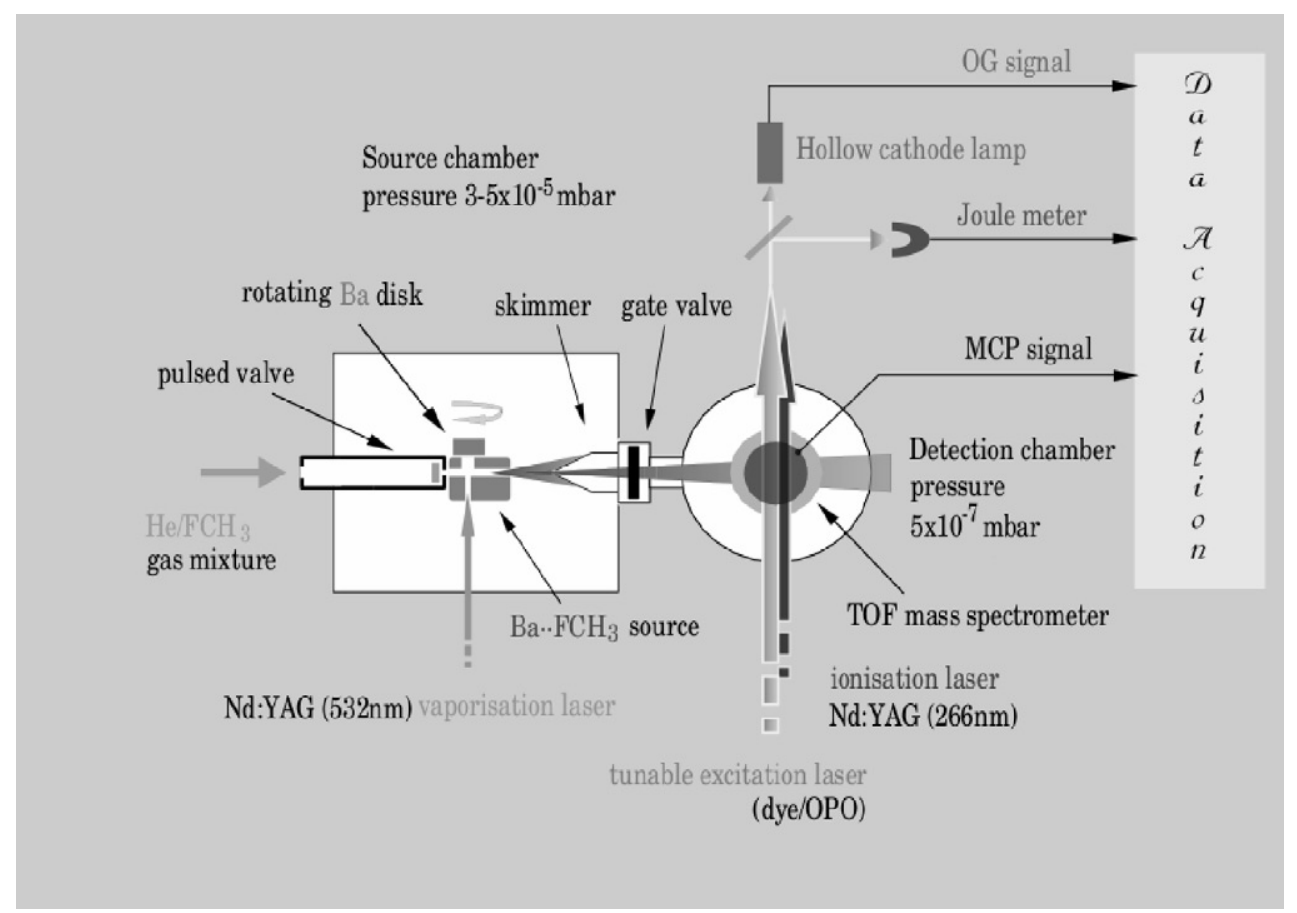

Figure 3. Experimental setup for a laser nanosecond pump and probe technique using cluster beams produced by laser vaporization methods. Adapted from [128].

ionization. A tuneable dye laser with a bandwidth of $0.08 \mathrm{~cm}^{-1}$ is used to excite the $\mathrm{Ba} \cdot \cdots \mathrm{FCH}_{3}$ complex in the desired wavelength range. The ionization laser is delayed by about $10 \mathrm{~ns}$ with respect to the excitation laser. Wavelength calibration was achieved using a standard hollow-cathode spectral lamp ( $\mathrm{Sn} / \mathrm{Ne}$, Cathodeon).

Care is taken to keep the excitation laser energy within a regime where the BeerLambert law can be applied. A key feature of the experimental technique is the simultaneous measurement of both the complex photo-depletion and the product action spectra.

The molecular beam extracted by a skimmer is interrogated in two different ways. Firstly, a Nd:YAG laser is used to ionize the species of interest. Secondly, in order to carry out photo-depletion experiments, the second-harmonic output of the same Nd:YAG laser is split into two beams, of which one is doubled to give the $266 \mathrm{~nm}$ radiation for ionization, and the other pumps a dye laser (this laser induces the chemical reaction within the weakly bound complex).

Figure 4 displays the photo-depletion spectrum of the $\mathrm{Ba} \cdot \cdot \cdot \mathrm{FCH}_{3}$ cluster in its excited $A^{\prime}$ state. The complex is excited through the range $730-760 \mathrm{~nm}$ by laser pulses of nanosecond duration. The spectrum exhibits a structureless shape, consistent with direct photo-dissociation. As shown in figure 5, laser excitation pumps the cluster to a repulsive state that leads to $\mathrm{Ba}^{*}+\mathrm{FCH}_{3}$.

In many cases not only can the reagents' depletion be measured, but also the appearance of the product, i.e. an action spectrum. Figure 6 shows the $\mathrm{CaCl}^{*}$ action 


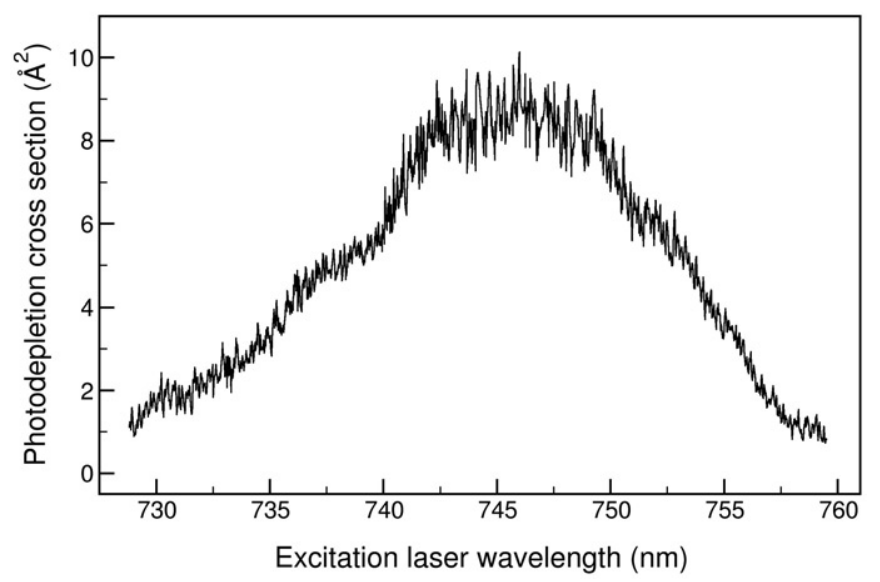

Figure 4. $\mathrm{Ba} \cdot \cdots \mathrm{FCH}_{3}$ photo-depletion spectrum, for excitation over the range $730-760 \mathrm{~nm}$. This figure is reproduced with permission from [135].

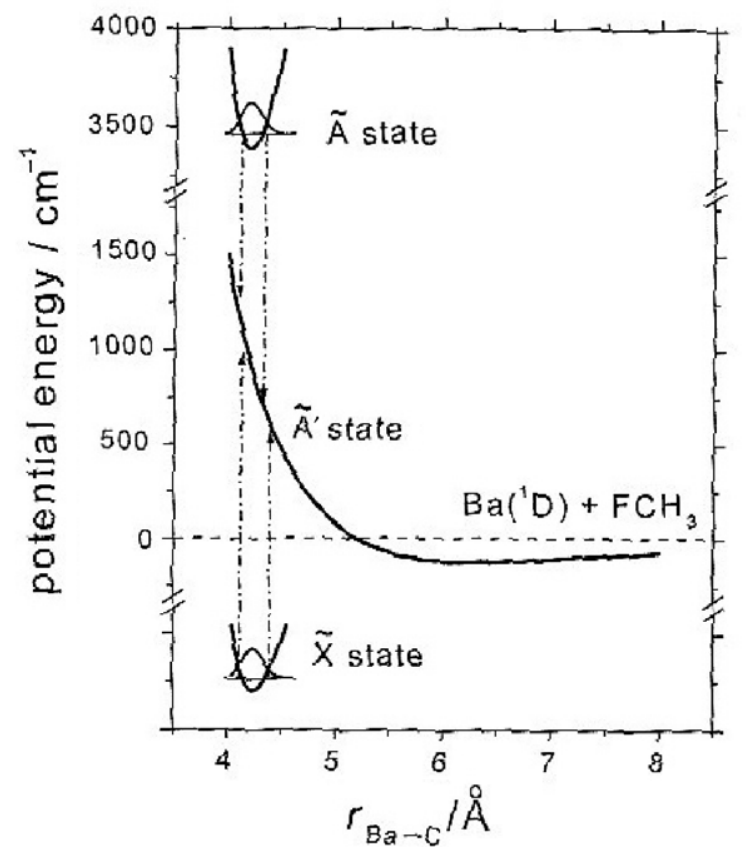

Figure 5. Ab initio potentials of $\mathrm{Ba} \cdot \mathrm{FCH}_{3}$ in different electronic states as indicated. Notice the repulsive character of the $A^{\prime}$ state. This figure is reproduced with permission from [130].

spectrum from the laser induced $\mathrm{Ca} \cdot \cdots \mathrm{HCl}$ intracluster reaction. The $\mathrm{Ca} \cdot \cdots \mathrm{HCl}$ complex is prepared in a supersonic expansion and excited close to the atomic resonance to a repulsive electronic state. Dissociation into $\mathrm{CaCl}$ and $\mathrm{H}$ can be characterized either by tuning the laser frequency and recording the subsequent action spectrum, or via LIF.

The example shown in figure 6 corresponds to $\mathrm{CaCl}^{*}$ chemiluminescence, which is recorded as a function of the (laser) excitation energy deposited on the $\mathrm{Ca} \cdot \cdots \mathrm{HCl}$ 

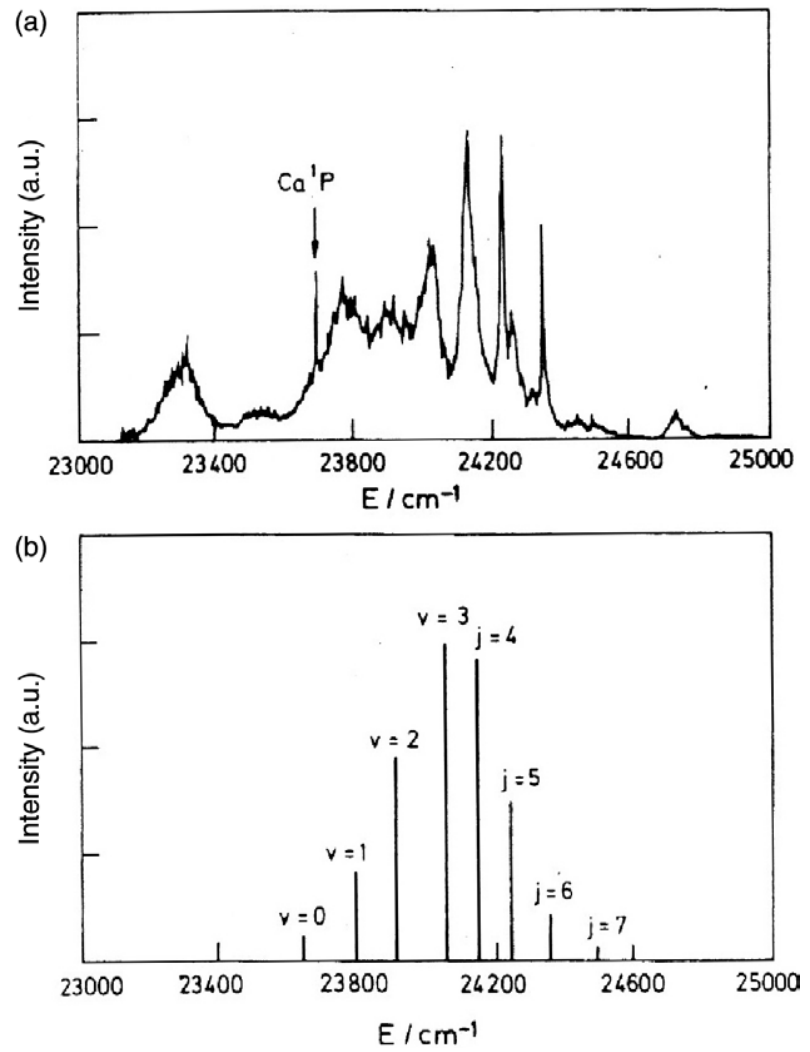

Figure 6. $\mathrm{CaCl}^{*}$ action spectrum from the laser $\mathrm{Ca} \cdot \cdots \mathrm{HCl}$ intracluster reaction. (a) Experimental results. (b) Calculated lines using a bending model for the transition state motion. This figure is reproduced with permission from [124].

cluster. It is a typical action spectrum. The different peaks were assigned and interpreted as local mode excitations, e.g. excitation of perpendicular motions to the reaction coordinate. Bending modes or free rotation excitation of the van der Waals $\mathrm{Ca} \cdot \cdots \cdot \mathrm{HCl}$ complex can be interpreted as belonging to this reactive channel yielding $\mathrm{CaCl}^{*}$.

The bottom part of figure 6 displays the action spectrum calculated using a bending model, which was adjusted to reproduce the position of the lines of the top (experimental) spectrum. From this analysis the main features of the excited bending potential can be deduced, i.e. the potential well as well as the potential barrier for free $\mathrm{HCl}$ rotation in the excited $\mathrm{Ca} \cdot \cdots \mathrm{HCl}$ state.

\section{Full collision studies}

\subsection{Gas cell studies by the pump-probe technique}

Activation of $\mathrm{H}-\mathrm{H}, \mathrm{C}-\mathrm{H}$, and $\mathrm{C}-\mathrm{C}$ bonds by metal elements has attracted wide attention due to their importance to catalytic and atmospheric chemistry and fuel exploration. For practical applications, metal hydrides are potential candidates for energy storage 
of hydrogen. Alkaline-earth hydrides used as decomposable hydrides for alkaline fuel cells are recommended for their ultimately high yields of hydrogen generation after hydrolysis [33]. The reverse reactions between alkaline-earth atoms and molecular hydrogen, $\mathrm{M}+\mathrm{H}_{2} \rightarrow \mathrm{MH}+\mathrm{H}$, are highly endoergic in their ground state, requiring a large deposit of energy to initiate the reaction. This is easily achieved via electronic excitation of the atom. Then, non-adiabatic couplings necessarily occur along the reaction path, as the $\mathrm{M}+\mathrm{H}_{2}$ ground state potential surface is adiabatically correlated to the $\mathrm{MH}+\mathrm{H}$ ground state potential surface. On the other hand, the reaction involves only limited numbers of atoms and active electrons, which makes possible for realistic, accurate calculations of potential energy surfaces (PES) and collision dynamics, even in the excited states that are concerned. Therefore, these photo-chemical reactions serve as promising candidates for detailed, generalized studies of non-adiabatic processes in reaction dynamics. In the following we focus on the $\mathrm{Mg}$ and $\mathrm{Ca}$ reactions with $\mathrm{H}_{2}$, followed by the reactions with $\mathrm{CH}_{4}$.

3.1.1 $\mathrm{Mg}+\mathbf{H}_{2}$. The $\mathrm{Mg}\left(3 \mathrm{~s}^{1} \mathrm{~S}_{0}\right)+\mathrm{H}_{2}$ reaction is endoergic by $27666 \mathrm{~cm}^{-1}$. Upon excitation at $285 \mathrm{~nm}$, the $\mathrm{Mg}\left(3 \mathrm{~s} 3 \mathrm{p}^{1} \mathrm{P}_{1}\right)+\mathrm{H}_{2}$ reaction becomes exoergic by $7340 \mathrm{~cm}^{-1}$, leading to the $\operatorname{MgH}(v, N)$ product [6-8, 34-43]. The rotational state distribution shows a bimodal feature with a major large- $N$ component and a minor low- $N$ component. The $\mathrm{Mg}\left(3 \mathrm{p}^{1} \mathrm{P}_{1}\right)-\mathrm{H}_{2}$ reaction is dominated by an insertion process tracking along the attractive ${ }^{1} \mathrm{~B}_{2}\left(\mathrm{C}_{2 v}\right.$ symmetry) or ${ }^{1} \mathrm{~A}^{\prime}\left(\mathrm{C}_{s}\right.$ symmetry) curve, whereas the colliding species in a collinear approach is obscured by a substantial potential barrier as high as 30-41 kcal mol ${ }^{-1}[40,43]$. Breckenridge and Wang have observed an isotope effect on the $\mathrm{MgH}$ distribution and Lin and Huang recorded the temperature dependence $[35,41]$. Their observations support the view that the product rotational bimodality should be attributed to two distinct microscopic pathways occurring in the exit channel. As $\mathrm{Mg}\left(3 \mathrm{p}^{1} \mathrm{P}_{1}\right)$ approaches toward $\mathrm{H}_{2}$ in $\mathrm{C}_{2 v}$ symmetry, the atomic orbital splits to three curves, ${ }^{1} \mathrm{~A}_{1},{ }^{1} \mathrm{~B}_{1}$, and ${ }^{1} \mathrm{~B}_{2}$, of which the ${ }^{1} \mathrm{~B}_{2}$ surface crosses the ground state surface [34, 40, 43].

After the non-adiabatic transition, the $\mathrm{MgH}_{2}$ collision complex decomposes on the anisotropic ground state surface. Inspecting the related dynamical potential energy surfaces for the first excited and the ground states may shed light on the microscopic pathways leading to the rotational bimodality [43]. The high- $N$ component, affected by the strong anisotropy, is formed via a collinear collision complex before dissociating. In contrast, the low- $N$ component is subject to a weaker anisotropic interaction; the departing $\mathrm{H}$ atom leaves along the $\mathrm{Mg}-\mathrm{H}$ stretching coordinate at a rate faster than that for the change of the $\mathrm{HMgH}$ angle. The subsequent small rotational torque leads to low- $N$ formation. The above comprehension of the reaction pathways is based on features of the coupling strength between the final states and the exit channel potential [44]. However, a quantitative analysis of the measured distributions demands dynamical calculations.

Quasi-classical trajectory (QCT) calculations were carried out using the corresponding fit functions and resulted in the following outcomes [45, 46]. The rotational populations of $\mathrm{MgH}$ in the $v=0$ and 1 levels are characteristic of a bimodal distribution. The minor low- $N$ component of $\mathrm{MgH}(v=0)$ peaks at $N=11$, while the 
major high- $N$ component peaks at $N=29$. The ratio of their peak heights is $\sim 1: 2$, which is consistent with the experimental findings [7, 8, 35, 42].

Assuming the individual rotational intensity of $\mathrm{MgH}$ up to $N=30$, the corresponding vibrational populations for the $v=1$ and 0 levels yield a ratio of 0.52 , which is within the range of the observation $0.7 \pm 0.2[7,8]$. As $N>30$, the experimental rotational spectra become congested and inseparable.

While inspecting individual trajectories, as shown in figure 7, two aspects may be discerned. First, the $\mathrm{MgH}_{2}$ collision complex for the high- $N$ component is much more long-lived than that for the low- $N$ component. Second, for the high- $N$ trajectory, the bending angle reaches $180^{\circ}$ rapidly, whereas for the low- $N$ trajectory the bending angle expands only to $\sim 90^{\circ}$ when the departing $\mathrm{H}$ atom is far from the $\mathrm{MgH}$ product. This supports the interpretation for the bimodal rotational distributions based on the PES information.

The reaction mechanisms for the higher states of metal atoms become more complicated, since they usually involve multiple surface crossings and the $a b$ initio calculations for these higher states are less accurate. Meanwhile, an inherent difficulty linked to this type of experiment lies in the fact that the product contribution from those lower states, relaxed via collision or radiative cascade, may interfere with the state of interest. A quantitative measure of such product interference from other excited states was done in the $\mathrm{Mg}\left(4 \mathrm{~s}^{1} \mathrm{~S}_{0}, 3 \mathrm{~d}^{1} \mathrm{D}_{2}\right)+\mathrm{H}_{2}$ reactions by looking at the influence of the lower $3 p{ }^{1} \mathrm{P}_{1}$ state [47-49]. This was realized by use of a third pulsed laser beam to deplete the $3 \mathrm{p}\left({ }^{1} \mathrm{P}_{1}\right)$ population by exciting the $3 \mathrm{p}\left({ }^{1} \mathrm{P}_{1}\right) \rightarrow 6 \mathrm{~d}\left({ }^{1} \mathrm{D}_{2}\right)$ transition. A substantial fraction of the $3 p\left({ }^{1} P_{1}\right)$ population was pumped out to the $6 d\left({ }^{1} D_{2}\right)$ state whose lifetime is longer than the time needed for $\mathrm{MgH}$ formation, so that increasing the intensity of the depletion laser decreased the yield of $\mathrm{MgH}$. The influence of the $3 \mathrm{p}\left({ }^{1} \mathrm{P}_{1}\right)$ population on the $\mathrm{MgH}$ product distribution was thus determined directly without involving any complicated evaluation. Furthermore, a harpoon mechanism was proposed for these high-lying state reactions to account for the distinct difference in the collisional deactivation crosssections measured for various $\mathrm{Mg}$ excited states. As $\mathrm{Mg}\left(4 \mathrm{~s}^{1} \mathrm{~S}_{0}, 3 \mathrm{~d}^{1} \mathrm{D}_{2}\right)$ approaches $\mathrm{H}_{2}$, the collision complex first crosses to the ion-pair reaction coordinate; a series of diabatic curve crossings are then found along the $\mathrm{Mg}^{+} \mathrm{H}_{2}^{-}$coordinate, and finally one to the reactive ${ }^{1} \mathrm{~B}_{2}$ surface, which correlates with the $\operatorname{Mg}\left(3 \mathrm{p}^{1} \mathrm{P}_{1}\right)$ state [49].

Electronic excitation of the atom is an efficient way to meet the energy requirement for the endoergic reactions, $\mathrm{M}+\mathrm{H}_{2} \rightarrow \mathrm{MH}+\mathrm{H}$, to initiate the reaction. Nevertheless, energy deposited in various degrees of freedom may result in different dynamical complexity. For instance, the reaction of $\mathrm{O}\left({ }^{1} \mathrm{D}\right)$ and $\mathrm{H}_{2}$ favours an insertion mechanism [49]. But when energy greater than $1.8 \mathrm{kcal} \mathrm{mol}^{-1}$ is deposited in translation, a new channel of $\mathrm{H}$-abstraction reaction along a collinear configuration may be opened $[50,51]$. Again, energy deposited in the $v=1$ vibrational level of $\mathrm{H}_{2}$ may enhance the probabilities for the reaction pathways which are otherwise negligible in the $\mathrm{H}_{2}(v=0)$ reactions [52]. Like this case, the vibrational excitation of $\mathrm{H}_{2}$ helps open up new reaction pathways in the reactions with $\mathrm{C}\left({ }^{1} \mathrm{D}\right)$ and $\mathrm{O}\left({ }^{3} \mathrm{P}\right)[53,54]$ or increased rotational excitation of $\mathrm{HD}(v=1)$ in the reaction with $\mathrm{D}[25,55]$. An active focus to find how energy is deposited and released was carried out in the $\mathrm{Mg}\left(3 \mathrm{p}^{1} \mathrm{P}\right)+\mathrm{H}_{2}(v=1)$ reaction [24]. Hydrogen molecules in $v=1, j=0-3$ are prepared by using stimulated Raman pumping (SRP) [22-25, 52-55]. 


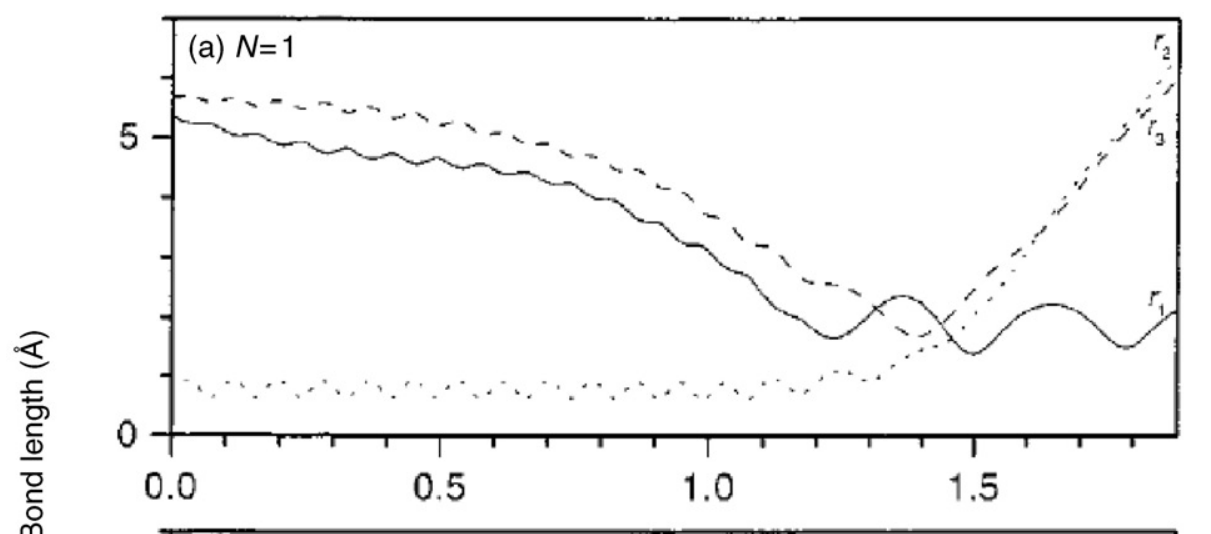

๓
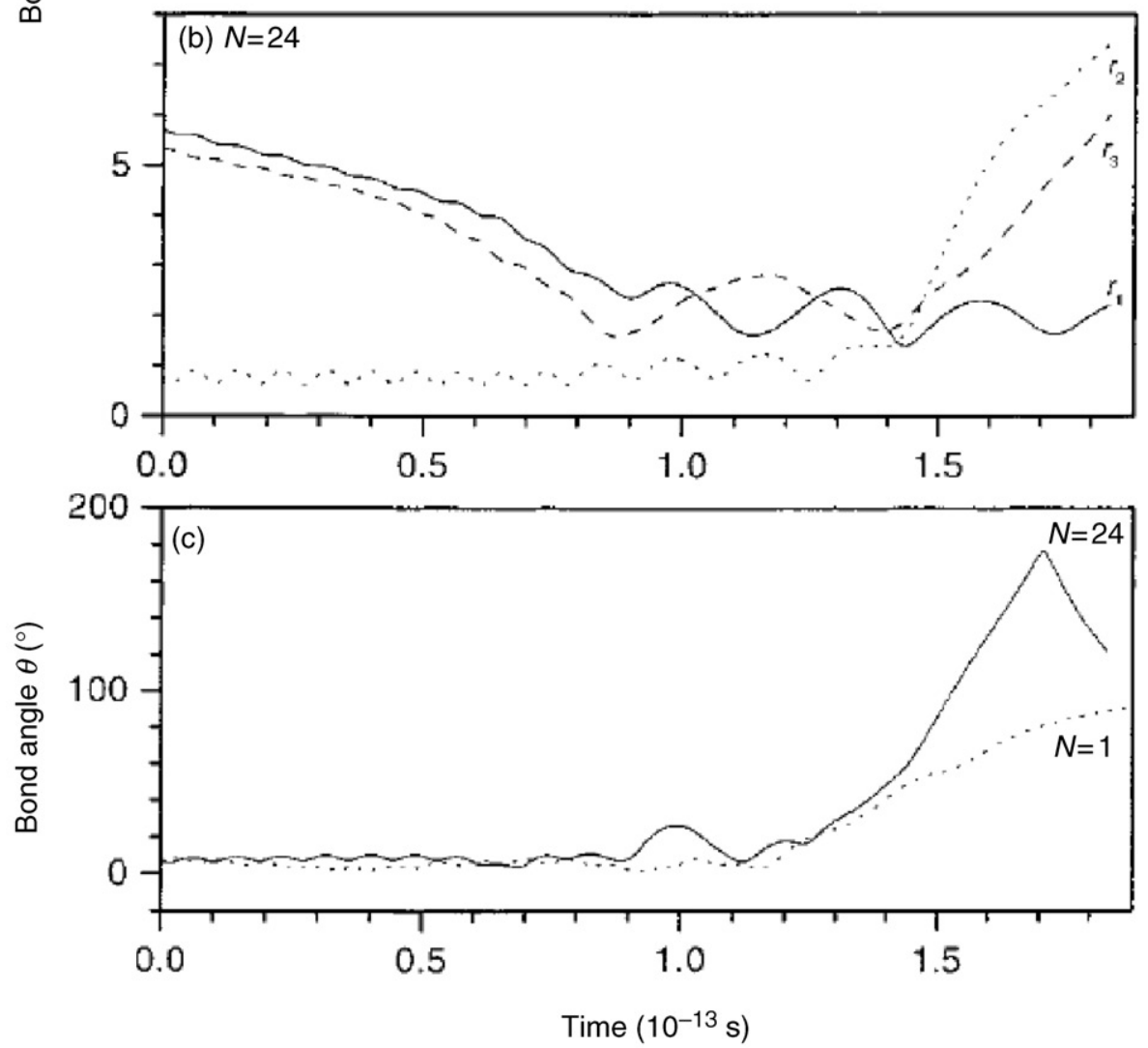

Figure 7. Evolution of trajectories for $\mathrm{MgH}(v=0, \mathrm{~N})$; (a) $N=1$ and (b) $N=24$. (c) Variation of the $\mathrm{H}-\mathrm{Mg}-\mathrm{H}$ angle. $r_{1}$ and $r_{3}$ indicate the distance between $\mathrm{Mg}$ and each $\mathrm{H}$, and $r_{2}$, the distance between two $\mathrm{H}$ atoms. This figure is reproduced with permission from [46].

A pump-probe technique is employed simultaneously to initiate reaction and monitor products. The population fraction in the $v=1$ level is estimated to be $7.5 \%$ by using coherent anti-Stokes Raman spectroscopy [22-24]. As shown in figure 8, vibrational excitation of $\mathrm{H}_{2}$ leads to enhancement in the low- $N$ 



Rotational quantum number

Figure 8. Rotational state distributions of $\mathrm{MgH}$ in the (a) $v=0$ and (b) $v=1$ levels in the $\operatorname{Mg}\left(3{ }^{1} \mathrm{P}_{1}\right)+\mathrm{H}_{2}$ reactions without stimulated Raman pumping (SRP) (denoted by open circles) and with SRP (denoted by filled circles). This figure is reproduced with permission from [24].

component of the bimodal rotational distribution and the vibration population in the $v=0$ level, but supression in the $v=1$ population. These features can be qualitatively predicted by using the QCT calculations on the reaction of $\operatorname{Mg}\left(3 p^{1} \mathrm{P}_{1}\right)$ with $\mathrm{H}_{2}(v=1, j=1)$.

By summing the reactive trajectories out of a total of 50000 , the fraction of low- $N$ component $(v=0, N<20)$ and the $v=0$ population are enhanced by factors of 1.3 and 1.1 , respectively, while the $v=1$ population is reduced by 0.83 (figure 9). These results can be comprehended with a kinematic collision model. Assume an extreme case for the vibrationally excited $\mathrm{H}_{2}$ molecule. That is, when $\mathrm{H}_{2}$ is excited, the $\mathrm{H}-\mathrm{H}$ bond stretches to become decoupled. As $\mathrm{Mg}$ attacks one $\mathrm{H}$ atom, the other $\mathrm{H}$ atom acts like a spectator without imposing any influence on the $\mathrm{MgH}$ to be formed.

The collision of a heavy atom toward a light one may kinematically tend to form the product with low vibrational and low rotational levels. This rationalizes why the 

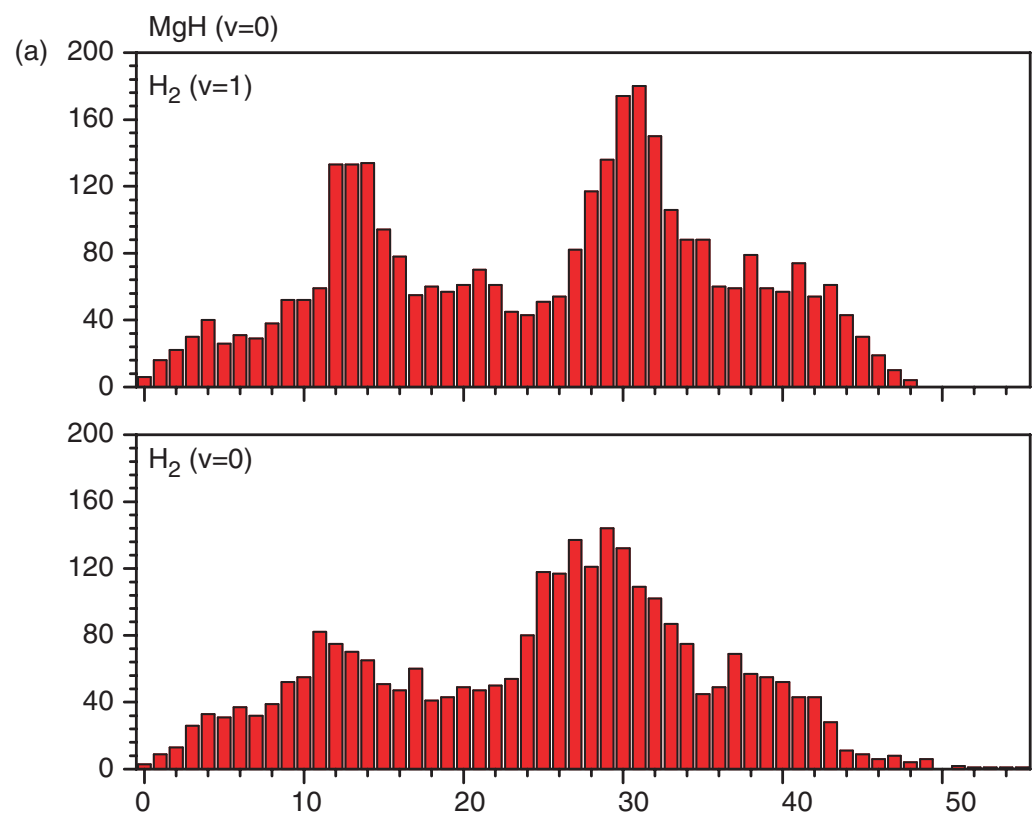

(b)
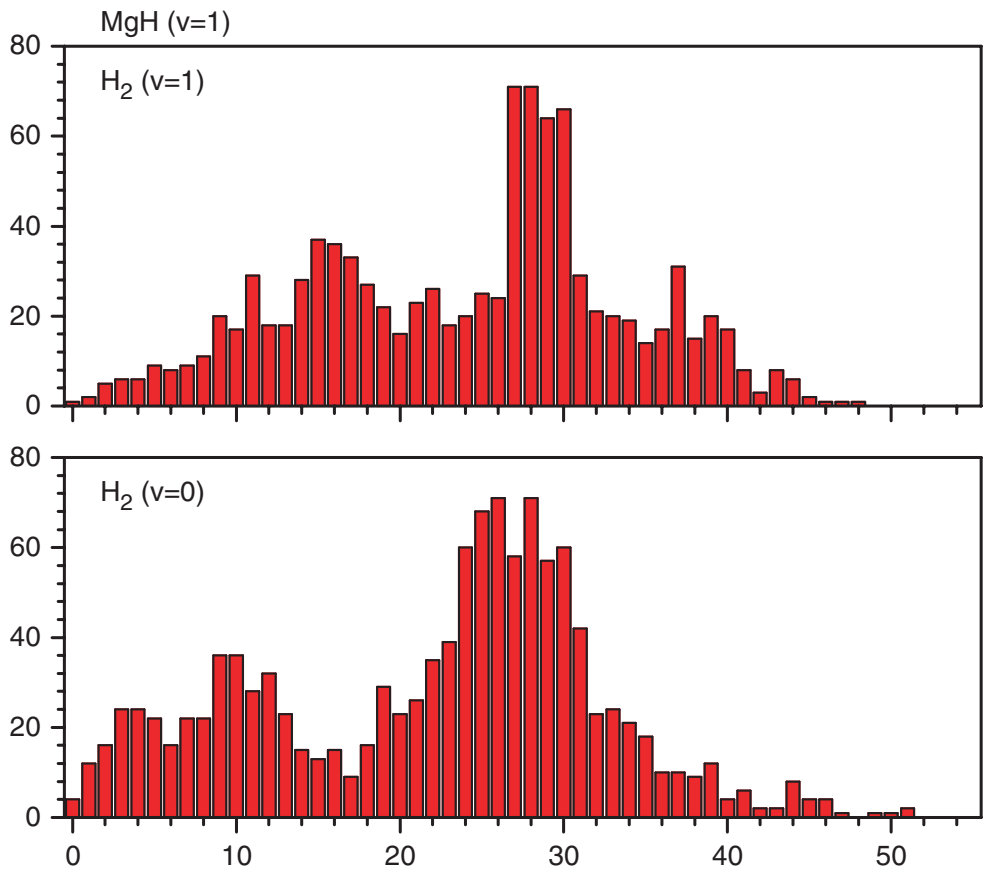

Figure 9. Rotational state distributions of $\mathrm{MgH}$ in the (a) $v=0$ and (b) $v=1$ levels simulated by the quasi-classical trajectory (QCT) model for the reactions of $\mathrm{Mg}\left(3 \mathrm{p}^{1} \mathrm{P}_{1}\right)$ with $\mathrm{H}_{2}(v=0, j=1)$ and $\mathrm{H}_{2}(v=1$, $j=1)$. The initial kinetic energy is $2.026 \mathrm{kcal} \mathrm{mol}^{-1}$. This figure is reproduced with permission from [24]. 
$\mathrm{MgH}(v=0) / \mathrm{MgH}(v=1)$ ratio and the low- $N$ products are enhanced. In contrast, in the $\mathrm{H}_{2}(v=0)$ case the coupling of $\mathrm{H}$ with $\mathrm{MgH}$ may cause the product to form in higher vibrational and higher rotational levels. The vibrational excitation of $\mathrm{H}_{2}$ has an impact on the entrance-channel potential surface, causing changes in the reaction cross-sections but without opening new reaction channels.

3.1.2 $\mathrm{Ca}+\mathrm{H}_{2}$. The $\mathrm{Ca}\left(4 \mathrm{~s}^{1} \mathrm{~S}_{0}\right)+\mathrm{H}_{2}$ reaction is endoergic by $22363 \mathrm{~cm}^{-1}$. The endoergicity is reduced to $513 \mathrm{~cm}^{-1}$ for the lowest singlet excited state $\mathrm{Ca}\left(4 \mathrm{~s} 3 \mathrm{~d}^{1} \mathrm{D}_{2}\right)$. A pump-probe technique was applied to initiate the $3 \mathrm{~d}^{1} \mathrm{D}$ reaction via two-photon absorption at $915 \mathrm{~nm}$ and then probe the $\mathrm{CaH}\left(\mathrm{X}^{2} \Sigma^{+}, v, N\right)$ product using LIF [21]. The nascent $\mathrm{CaH}(v=0,1)$ products are characterized by single-peaked Boltzmann rotational distributions. While taking into account the kinetic energy spread for the system temperature, the fractions of the available energy partitioned into rotation, vibration, and translation are estimated to be $0.36 \pm 0.05,0.28 \pm 0.04$, and $0.36 \pm 0.05$, respectively.

For the temperature dependence measurements, Arrhenius plots yield positive slopes, indicative of the reaction occurrence without a potential barrier. According to the PES calculations $[21,56]$, the colliding species are anticipated to follow an attractive $1 \mathrm{~B}_{2}$ (or $2 \mathrm{~A}^{\prime}$ ) surface favouring a near $\mathrm{C}_{2 v}$ collision configuration and then transit nonadiabatically to the reactive ground state surface. Assuming the three-atomic system lies on the $y z$ plane, the involvement of partial $\mathrm{Ca}^{+} \mathrm{H}_{2}^{-}$ion-pair character and the electron back-donation from the singly occupied $3 \mathrm{~d}_{y z}$ orbital of the $\mathrm{Ca}$ atom to the anti-bonding $\sigma^{*}$ of the molecular hydrogen may stabilize the corresponding $1 \mathrm{~B}_{2}$ (or $2 \mathrm{~A}^{\prime}$ ) surface at short range. This makes this PES the only one that may cross the ground PES.

The lowest excited singlet $\mathrm{P}$ state $\mathrm{Ca}\left(4 \mathrm{p}^{1} \mathrm{P}_{1}\right)$ (electronically excited with a laser pulse at $422.7 \mathrm{~nm}$ ) can result in reaction with exothermicity of $1267 \mathrm{~cm}^{-1}[57,58]$. The nascent product $\mathrm{CaH}\left(\mathrm{X}^{2} \Sigma^{+}\right)$was found to show a single-peaked distribution, as characterized by low rotational and high vibrational temperatures given a total available energy of $2893 \mathrm{~cm}^{-1}$, the fractions of energy released as rotation, vibration, and translation are $0.19 \pm 0.02,0.33 \pm 0.02$, and $0.48 \pm 0.02$, respectively [57].

From an Arrhenius plot, the temperature dependence measurement yields a barrier of $3820 \pm 480 \mathrm{~cm}^{-1}$. As a result of the potential energy surfaces (PES) calculations, the reaction pathway favours a Ca-insertion into the $\mathrm{H}_{2}$ bond along a (near) $\mathrm{C}_{2 v}$ geometric approach. The potential surfaces corresponding to the $3 \mathrm{~d}^{1} \mathrm{D}$ and $4 \mathrm{p}^{1} \mathrm{P}$ states are split into the $2-4 \mathrm{~A}^{\prime}$ and $5-6 \mathrm{~A}^{\prime}$ surfaces, respectively. If the colliding species along the $5 \mathrm{~A}^{\prime}$ surface crosses to the $4 \mathrm{~A}^{\prime}$ surface in the long range with the surface transition probability enhanced by the involvement of $\mathrm{Ca}^{+} \mathrm{H}_{2}^{-}$ion-pair character, then reaction might occur along the reactive $1 \mathrm{~A}^{\prime}$ surface without any barrier. This is not consistent with the experimental findings. The $5 \mathrm{~A}^{\prime} \rightarrow 4 \mathrm{~A}^{\prime}$ transition may not happen at short range either, because a repulsion of $1.5 \mathrm{eV}$ is too large for the colliding species to surmount.

From theoretical considerations, Kim et al. expected that the above reaction may not occur in a single collision event, because of the high energy barrier with $\mathrm{H}_{2}$ fixed at $0.75 \AA$ [56]. Nevertheless, as the $\mathrm{H}_{2}$ bond is elongated to $0.9 \AA$, the energy barrier is reduced substantially and further configurational mixing between the orbital components of the $4 \mathrm{p}$ and $3 \mathrm{~d}$ state with the same symmetry facilitates non-adiabatic 
transition between $5^{1} \mathrm{~A}^{\prime}$ ( or $2^{1} \mathrm{~B}_{2}$ ) and $2^{1} \mathrm{~A}^{\prime}$ ( or $1^{1} \mathrm{~B}_{2}$ ) surfaces in the repulsive limbs $[58,59]$. Thus, reactive collisions may take place only when $\mathrm{H}_{2}(v=0)$ stretches to its outer turning point. The observed energy barrier probably accounts for the energy requirement to surmount the repulsive hill in the entrance channel.

As the energy is first deposited in the vibrational levels of $\mathrm{H}_{2}$, enlargement of the $\mathrm{H}_{2}$ bond distance in the entrance channel may facilitate the reaction and increase the product yield. The $\mathrm{Ca}\left(4 \mathrm{p}^{1} \mathrm{P}\right)+\mathrm{H}_{2}(v=1)$ reaction was further conducted to confirm the proposed reaction pathway. The $\mathrm{H}_{2} v=1, j=0-3$ level was excited using stimulated Raman pumping, while the fraction of vibrational population in this level was determined to be $7.5 \%$ out of the total vibrational population by using coherent anti-Stokes Raman spectroscopy (CARS) [22-24]. As shown in figure 10, the resulting $\mathrm{CaH}(v=0$ and 1$)$ rotational distributions obtained by the $\mathrm{H}_{2}(v=1)$ reaction appeared to be similar to those by $\mathrm{H}_{2}(v=0)$ reaction, but the $\mathrm{CaH}$ product yield (or the reaction cross-section) was enhanced by an order of magnitude. Because of the similarity of rotational distributions between these two cases, the vibrational excitation may not significantly change the effective collisional configuration.

The lack of detection of $\mathrm{CaH}(v \geq 2)$ suggests that the additional energy of $4154 \mathrm{~cm}^{-1}$ is not distributed statistically among different degrees of freedom. The energy deposited in the vibration does not open up any additional reaction pathway to make the product distributions otherwise different.

The collisions of $\mathrm{Ca} 4 \mathrm{p}^{1} \mathrm{P}_{1}$ and $3 \mathrm{~d}^{1} \mathrm{D}$ atoms with $\mathrm{H}_{2}$ follow different reaction pathways. The colliding species in the former reaction has to surmount a potential barrier and then transit to the reactive ground state surface. Accordingly, the 'early' barrier existing in the entrance channel favours product vibrational excitation [60]. On the contrary, for $\mathrm{Ca}\left(3 \mathrm{~d}^{1} \mathrm{D}\right)+\mathrm{H}_{2}$ the rotational excitation is larger than the prior rotational distribution of $2 / 7$, while the vibrational excitation is less. These results can be predicted with an impulsive model [44]. Furthermore, the lifetimes of the $\mathrm{Ca}\left(4 \mathrm{p}^{1} \mathrm{P}_{1}\right)-\mathrm{H}_{2}$ and $\mathrm{Ca}\left(3 \mathrm{~d}^{1} \mathrm{D}\right)-\mathrm{H}_{2}$ intermediate complexes, assumed to be at the $1 \mathrm{~B}_{2}-1 \mathrm{~A}_{1}$ curve crossing where the Ca atom is $1.5 \AA$ from the centre of $\mathrm{H}_{2}$ bond, are estimated to be 1.6 and $14 \mathrm{ps}$, respectively [21]. The slow dissociation of the $\mathrm{Ca}\left(3 \mathrm{~d}^{1} \mathrm{D}\right)-\mathrm{H}_{2}$ complex favours that of internal energy dissipation. Thus, the product energy disposal is close to that of predicted by a statistical distribution.

It is also worthwhile to note the difference of internal energy distributions between $\mathrm{Mg}$ and $\mathrm{Ca}$ reactions. In the case of $\mathrm{Mg}\left(3 \mathrm{p}^{1} \mathrm{P}_{1}\right)$, the product $\mathrm{MgH}$ exhibits a bimodal rotational distribution, which is subject to the exit-channel anisotropic interaction on the ground state surface. In contrast, the $\mathrm{CaH}$ product energy distribution seems to reflect interactions originated at the PES entrance-channel.

3.1.3 $\mathrm{Mg}+\mathrm{CH}_{4}$. The interaction between alkaline-earth metals and alkane hydrocarbons is actively focused on the following reaction channel by using a pump-probe technique $[6-8,36,39,61-70]$.

$$
\mathrm{M}^{*}\left(\operatorname{nsnp}^{1,3} \mathrm{P}\right)+\mathrm{RH} \rightarrow \mathrm{MH}(v, N)+\mathrm{R}
$$



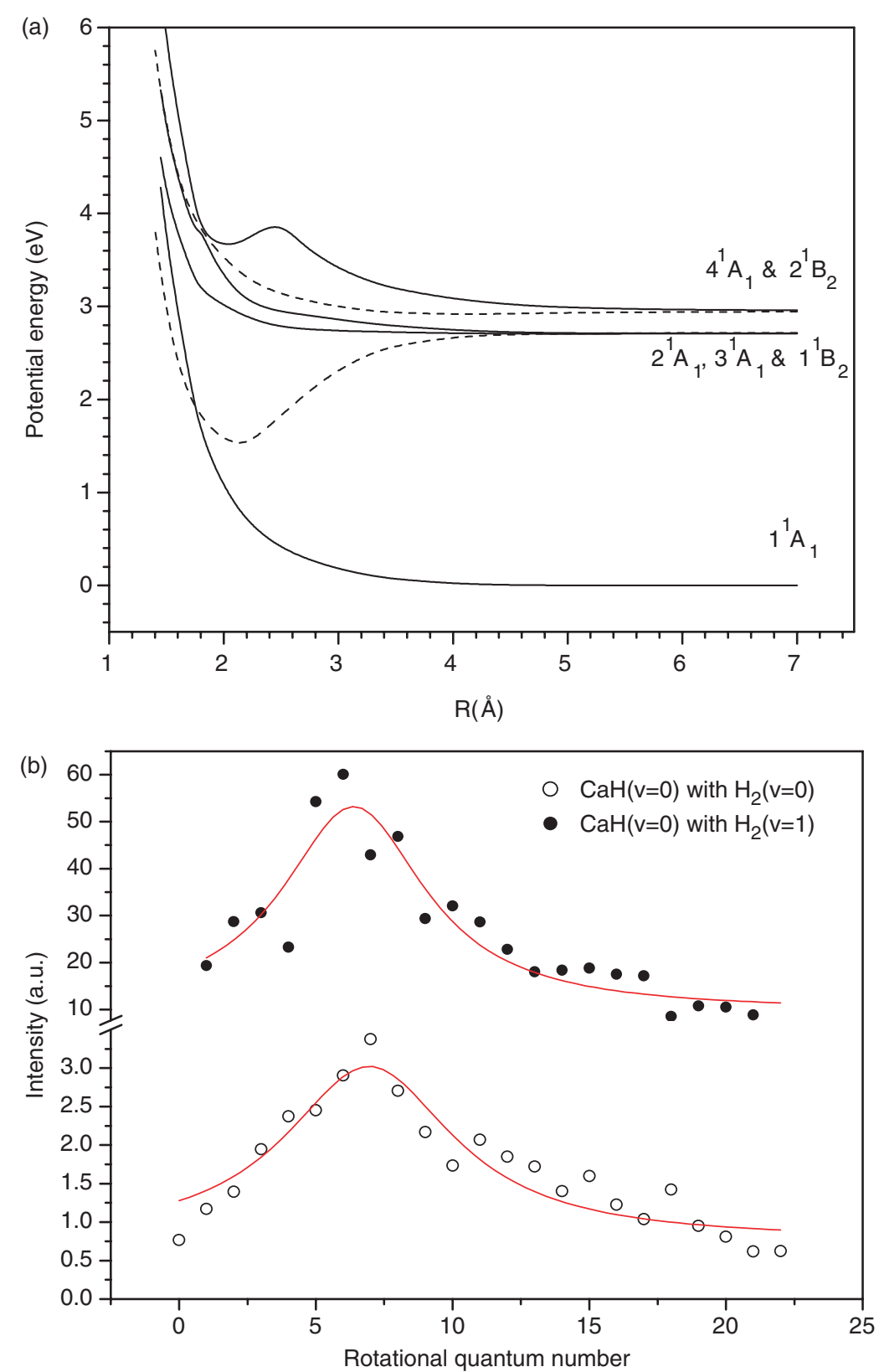

Figure 10. (a) Potential energies calculated with the multireference configuration interaction (MRCI) method for the $1-4^{1} \mathrm{~A}_{1}$ and $1-2^{1} \mathrm{~B}_{2}$ surfaces (or $1 \mathrm{~A}^{\prime}-6 \mathrm{~A}^{\prime}$ in $\mathrm{C}_{s}$ symmetry) as a function of the distance between $\mathrm{Ca}$ and the centre of $\mathrm{H}_{2}$ in $\mathrm{C}_{2 v}$ symmetry. The $\mathrm{H}_{2}$ bond is stretched to $0.9 \AA$. The dashed curves denote the $1^{1} \mathrm{~B}_{2}$ (or $2 \mathrm{~A}^{\prime}$ ) and $2^{1} \mathrm{~B}_{2}$ (or $5 \mathrm{~A}^{\prime}$ ) surfaces. (b) Rotational state distributions of $\mathrm{CaH}(v=0)$ in the reactions of $\mathrm{Ca}\left(4^{1} \mathrm{P}_{1}\right)$ with $\mathrm{H}_{2}(v=0)$ and $\mathrm{H}_{2}(v=1)$. This figure is reproduced with permission from [24]. 
The energy required to initiate the reaction can be deposited in the electronic states of the metal atom $\mathrm{M}^{*}$ with a pump-laser source. After a brief time delay the $\operatorname{MH}(v, N)$ product distributions are monitored with a probe laser, yielding nascent product distributions.

Among the alkaline-earth metals, $\mathrm{Mg}$ has been investigated thoroughly [6-8, 36, 39, 61-68]. Breckenridge and coworkers have studied $\mathrm{Mg}\left(3 \mathrm{p}^{1} \mathrm{P}_{1}\right)$ reactions with a series of alkane and alkene hydrocarbons ( $\mathrm{RH})$ since the early 1980s [6-8, 61, 62]. The $\mathrm{MgH}$ product rotational distributions appear to be bimodal whose rotational components seem independent of the hydrocarbon's complexity. They suggested that the intermediate $\mathrm{HMgR}$ should be short-lived enough to avoid vibrational energy flow into the alkyl portion of the intermediate [36]. Kleiber and coworkers affirmed the dominance of $\mathrm{Mg}$ insertion mechanism by using a far-wing scattering technique. The $\mathrm{MgH}$ low- $N$ and high- $N$ distributions are originated from the same mechanism controlled by anisotropy of the exit channel surface, but independent of how and where the $\mathrm{MgCH}_{4}$ complex is excited in the entrance channel [66]. Their conclusion is consistent with the temperature dependence measurements of Liu et al. [65] and the theoretical PES calculations of Chaquin et al. [63]. A substantial potential barrier of $35 \mathrm{kcal} \mathrm{mol}^{-1}$ may be encountered as $\mathrm{Mg}\left(3^{1} \mathrm{P}_{1}\right)$ approaches $\mathrm{CH}_{4}$ along a collinear $\mathrm{Mg}-\mathrm{H}-\mathrm{C}$ axis, whereas the insertion pathway has the smallest barrier $<6.9 \mathrm{kcal} \mathrm{mol}^{-1}$. This small barrier was not found in the experiments.

In the Arrhenius plot, a negative temperature dependence of the measurements of thermal rate coefficients of $\operatorname{Mg}\left(3 p{ }^{1} \mathrm{P}_{1}\right)$ indicates an attractive surface with a 5.75$6.54 \mathrm{kcal} \mathrm{mol}^{-1}$ intermediate well located in the surface crossing between the excited and the ground states [68]. The measurements also reveal the dominance of the chemical reaction over the $\mathrm{Mg}\left(3 \mathrm{p}^{1} \mathrm{P}_{1}\right)$ depletion channels by the $\mathrm{CH}_{4}$ collisions.

According to the PES calculations, the $\mathrm{Mg}-\mathrm{C}$ distance for $\mathrm{HMgCH}_{3}$ in the transition state is $2.76 \AA$ [63], about $31.3 \%$ longer than its equilibrium distance $2.102 \AA$ [71]. The orbital overlap between $\mathrm{Mg}$ and $\mathrm{CH}_{4}$ is inefficient such that the $\mathrm{Mg}-\mathrm{C}$ bond strength becomes weak and the subsequent $\mathrm{MgCH}_{3}$ product cannot be observed for this reaction. Since the coupling between $\mathrm{MgH}$ and $\mathrm{CH}_{3}$ is weak, slow energy transfer between them preserves the bimodal $\mathrm{MgH}$ product distributions.

In a two-dimensional dynamical PES calculation, Liu et al. further suggested that the $\mathrm{MgH}$ distribution depends on anisotropic interaction of the final state in the exit channel following a non-adiabatic transition [65]. The high- $N$ states were anticipated to result from the pathway that the $\mathrm{HMgCH}_{3}$ collision complex evolves through a $\mathrm{C}_{3 v}$ configuration before decomposition, whereas the low- $N$ states are formed through the pathway with weaker angular dependence of the potential interaction. A triplet intermediate is not involved in the $\operatorname{Mg}\left(3{ }^{1} \mathrm{P}_{1}\right)$ reaction such that the atomic emission from the triplet state has not been observed [6-8, 61, 62]. Nevertheless, the triplet-state emission becomes detectable with the use of Xe-matrix isolation at $12 \mathrm{~K}$ [72-74], since the spin-orbit coupling may be enhanced due to the external heavy atom effect.

3.1.4 $\mathbf{C a}+\mathbf{C H}_{4}$. Although the elements $\mathrm{Mg}$ and $\mathrm{Ca}$ belong to the same group, their reactions with $\mathrm{CH}_{4}$ exhibit marked differences in dynamical behaviour. When $\mathrm{Ca}\left(4 \mathrm{p}^{1} \mathrm{P}_{1}\right)$ is substituted for $\mathrm{Mg}\left(3 \mathrm{p}^{1} \mathrm{P}_{1}\right)$, the reaction with exothermicity of $1052 \mathrm{~cm}^{-1}$ 
is made more complicated by involvement of multiple PESs. In terms of a pump-probe technique, the $\mathrm{CaH}(v=0$ and 1) product distributions are found to be single-peaked [23]. The rotational population may be fitted by a statistical prior distribution [75], showing a feature of low Boltzmann rotational temperature. The rotational and vibrational energy in $\mathrm{CaH}$ was estimated to be $555 \pm 22$ and $995 \pm 10 \mathrm{~cm}^{-1}$, respectively, corresponding to fractions of 0.19 and 0.33 , for a total available energy for dissipation of $2991 \mathrm{~cm}^{-1}$. The normalized rotational excitation increases with the increase of temperature, suggesting that there exists a reaction barrier estimated to be less than $4000 \mathrm{~cm}^{-1}$.

The PES calculations disfavour the reaction pathway to follow a $\mathrm{C}_{3 v}$ end-on attack to abstract an $\mathrm{H}$ atom. Similar conclusions may also be found in other cases such as the $\mathrm{Mg}\left(3^{1} \mathrm{P}\right), \mathrm{Zn}\left(4^{1} \mathrm{P}\right)$ [76-78], $\mathrm{Cu}\left(4^{2} \mathrm{P}\right)$ [79], $\mathrm{O}\left(2^{1} \mathrm{D}_{2}\right)$ [80-82], and $\mathrm{N}\left(2^{2} \mathrm{D}\right)$ [83] with $\mathrm{CH}_{4}$ reactions.

The reaction pathway favours an insertion mechanism, $\mathrm{Ca}\left(4{ }^{1} \mathrm{P}_{1}\right)$ approach to $\mathrm{CH}_{4}$ in $\mathrm{C}_{2 v}$ or $\mathrm{C}_{s}$ symmetry. After surpassing an entrance barrier, the colliding species may undergo a series of surface transitions to the reactive ground state surface. It may not be surprising to find the $\mathrm{CaH}$ product in a cool rotational distribution, different from the bimodal feature by the $\mathrm{Mg}\left(3 \mathrm{p}^{1} \mathrm{P}_{1}\right)$ reaction. The $\mathrm{Ca}-\mathrm{C}$ bond distance of the intermediate around the surface crossing region is $2.7-2.8 \AA$, about $14.9-19.2 \%$ longer than the equilibrium distance $2.349 \AA$ [84]. The strong coupling of the moieties through a tight overlap of the $\mathrm{Ca}-\mathrm{C}$ molecular orbital renders the energy transfer sufficient from $\mathrm{CaH}$ into the $\mathrm{CH}_{3}$ radical. As a result of the short $\mathrm{Ca}-\mathrm{C}$ distance, the collision complex may decompose to $\mathrm{CaCH}_{3}$, in addition to the main $\mathrm{CaH}$ product [70]. The intermediate complex with a small excess energy can also last long enough to dissipate excess energy efficiently before decomposition.

The exit channel of the $\mathrm{Ca}\left(4 \mathrm{p}^{1} \mathrm{P}_{1}\right)+\mathrm{CH}_{4}$ reaction is associated with the $\mathrm{Ca}\left(4 \mathrm{~s} 3 \mathrm{~d}^{1} \mathrm{D}_{2}\right)-$ $\mathrm{CH}_{4}$ potential surface (the lowest excited singlet state of $\mathrm{Ca}$ ). Even though the $\mathrm{Ca}\left(4 \mathrm{~s} 3 \mathrm{~d}^{1} \mathrm{D}_{2}\right)+\mathrm{CH}_{4}$ reaction is endothermic by $2930 \mathrm{~cm}^{-1}$, the $\mathrm{CaH}$ product can be readily obtained by using a pump-probe technique [68]. The nascent product distributions of $\mathrm{CaH}$ were characterized with a Boltzmann rotational temperature which was close to the oven temperature.

The rotational and vibrational energy partitions in $\mathrm{CaH}$ were estimated to be $461 \pm 45$ and $252 \pm 15 \mathrm{~cm}^{-1}$, respectively, corresponding to a fraction of 0.41 and 0.22 , given a total available energy for dissipation of $1127 \mathrm{~cm}^{-1}$. According to the PES calculations, the pathway favours an insertion mechanism. $\mathrm{Ca}\left(3 \mathrm{~d}^{1} \mathrm{D}_{2}\right)$ approaches $\mathrm{CH}_{4}$ along an attractive potential surface in $\mathrm{C}_{2 v}$ ( or $\mathrm{C}_{s}$ ) symmetry and then the collision complex undergoes non-adiabatic transition to the reactive ground state surface. An Arrhenius plot shows a potential energy requirement of $2695 \pm 149 \mathrm{~cm}^{-1}$, which accounts for the endothermicity for the reaction scheme (figure 11).

In the reaction of $\mathrm{Ca}\left(4^{1} \mathrm{P}_{1}\right)$ with $\mathrm{CH}_{4}$, an 'early' barrier in the entrance channel favours vibrational excitation [60]. It is reflected in the fact that a fraction up to $33 \%$ of the available energy is partitioned into vibration in the $\mathrm{Ca}\left(4 \mathrm{p}^{1} \mathrm{P}_{1}\right)$ reaction, but only $22 \%$ is obtained in the $\mathrm{Ca}\left(3^{1} \mathrm{D}_{2}\right)$ reaction. On the other hand, in estimating lifetimes of the intermediate complexes, $\mathrm{Ca}\left(3 \mathrm{~d}^{1} \mathrm{D}_{2}\right)-\mathrm{CH}_{4}$ is long-lived by an order of magnitude than $\mathrm{Ca}\left(4^{1} \mathrm{P}_{1}\right)-\mathrm{CH}_{4}$, and thus has a greater chance for internal energy dissipation. 


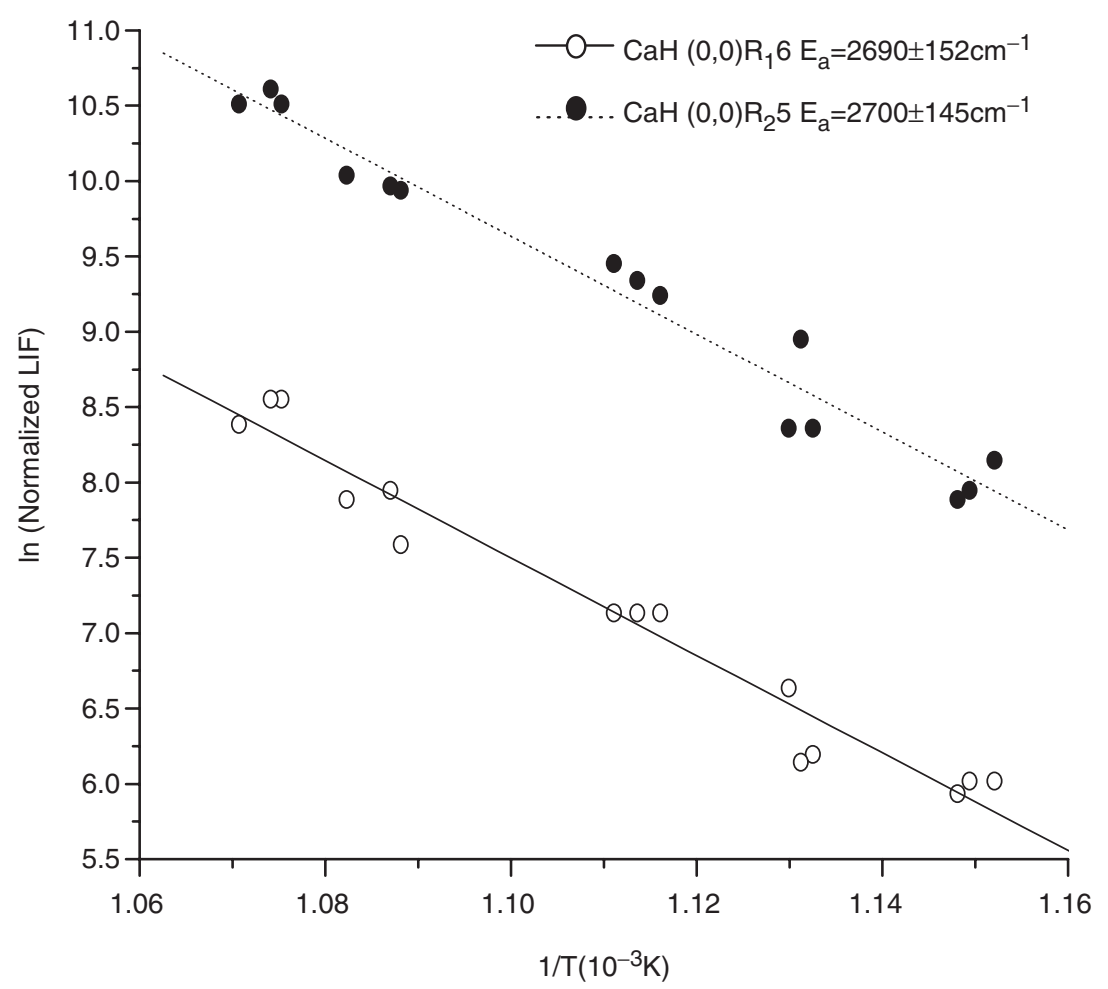

Figure 11. Arrhenius plot of normalized laser-induced fluorescence (LIF) of rotational lines $(0,0) \mathrm{R}_{2} 5$ and $(0,0) R_{1} 6$ in the reaction of $\mathrm{Ca}\left(3 \mathrm{~d}^{1} \mathrm{D}_{2}\right)$ with $\mathrm{CH}_{4}$ versus the reciprocal of temperature varied from 871 to $934 \mathrm{~K}$. The corresponding activation energies are determined to be $2700 \pm 145$ and $2690 \pm 152 \mathrm{~cm}^{-1}$, respectively. This figure is reproduced with permission from [70].

\subsection{Beam-gas studies: total reaction cross-section determined from beam-gas experiments}

In a beam-gas experiment one can measure the total beam attenuation as a function, for example, of the gas cell pressure. From this type of measurement it is not possible to deduce the total reactive cross-section as both reactive and non-reactive collisions contribute to the beam attenuation. Nevertheless, there are cases in which determination of the reaction cross-section is possible. In this section we shall discuss this possibility and present some examples of it.

Assuming that we have a beam of particles A whose intensity $I_{\mathrm{A}}$ is $v_{\mathrm{A}} \cdot c_{\mathrm{A}}$, i.e. its velocity times its concentration, colliding with a gas $\mathrm{B}$ which is inside a gas cell and with a concentration $\mathrm{c}_{\mathrm{B}}$. The differential beam intensity loss, $\mathrm{d} I_{\mathrm{A}}$ can be written as:

$$
-\mathrm{d} I_{\mathrm{A}}=\sigma_{\mathrm{AB}} \cdot I_{\mathrm{A}} \cdot C_{\mathrm{B}} \cdot \mathrm{d} l
$$

Here $\sigma_{\mathrm{AB}}$ is the so-called collision cross-section, $I_{\mathrm{A}}(1)$ means the intensity of $\mathrm{A}$ at a distance $1, I_{\mathrm{A}}(0)$ is the initial intensity, i.e. the intensity before A enters the gas cell, and 
$\mathrm{d} l$ is the collision path inside the gas cell. After integration of (3) one gets

$$
I_{\mathrm{A}}(l)=I_{\mathrm{A}}(0) e^{-\sigma_{\mathrm{AB}} c_{\mathrm{B}} l}
$$

Only a fraction of all collisions between $\mathrm{Ca}\left({ }^{3} \mathrm{P}_{\mathrm{J}}\right)+\mathrm{CH}_{3} \mathrm{I}$ will lead to a reaction that will produce $\mathrm{CaI}$ and, consequently, the reaction cross-section $\sigma_{\mathrm{R}}$ is undoubtedly smaller than $\sigma_{\mathrm{AB}}$.

Figure 12(a) upper panel, shows the image of a calcium beam moving inside a vacuum chamber. The emitted light corresponds to the transition $\mathrm{Ca}\left({ }^{3} \mathrm{P}_{1}-{ }^{1} \mathrm{~S}_{0}\right)$, that is forbidden by electric dipole interaction (this is why the excited calcium $\mathrm{Ca}{ }^{3} \mathrm{P}_{1}$ lives so long, i.e. $\tau \approx 340 \mu$ s so we can see it). Figure 12 (b) represents the same image but this was taken when a few mbar of $\mathrm{CH}_{3} \mathrm{I}$ was added to the chamber. Attenuation is evident.

One can plot the intensity of the calcium beam as a function of the distance in a semi$\log$ plot of $I_{\mathrm{Ca}^{*}}$ versus the attenuation path. From the slope an attenuation cross-section of $111 \AA^{2}$ was deduced.

The question now arises about whether it is possible to determine $\sigma_{\mathrm{R}}$ ? In general, the answer is negative but a few exceptions are possible. Suppose that we add $\mathrm{CH}_{3} \mathrm{I}$. Then the following reaction will take place, $\mathrm{Ca}\left({ }^{3} \mathrm{P}_{\mathrm{j}}\right)+\mathrm{CH}_{3} \mathrm{I} \rightarrow \mathrm{CaI}^{*}+\mathrm{CH}_{3}$ in which nascent $\mathrm{CaI}^{*}$ emits over the visible spectral range. Therefore, the chemiluminescence picture can be taken as in the previous experiment, but now with a different filter installed in front of the CCD camera. The interference filter was centred at $\lambda \cong 630 \mathrm{~nm}$ so that one could get rid of the metastable emission. In fact, what was done was to measure the emission profiles along the $\mathrm{Ca}$ beam axis of both metastable emission from the atomic beam and nascent $\mathrm{CaI}^{*}$ emission. The lifetime of the nascent product is well known to lie around $50 \mathrm{~ns}$ so that its diffusion out of the viewing zone can be ruled out.

For a bimolecular beam gas reaction one can write the chemiluminescence CaI intensity.

$$
I_{\text {chem }}=n_{g} V_{r} \frac{I_{3 \mathrm{P}}}{A_{1 \mathrm{P}} f_{J=1}} \sigma_{\mathrm{R}}
$$

in which $\mathrm{A}_{3 \mathrm{p}}$ is the Einstein coefficient for the forbidden ${ }^{3} \mathrm{P}_{1} \rightarrow{ }^{1} \mathrm{~S}_{0}$ electronic transition of calcium, $f_{J=1}$ is the $n_{1} /\left(n_{0}+n_{1}+n_{2}\right)$ fraction, $I_{3 \mathrm{P}}$ is the metastable emission, $\sigma_{\mathrm{R}}$ is the chemiluminescent reaction cross-section, and $\mathrm{Vr}$ is the relative velocity. Due to the linear dependence of the product emission and the CaI short lifetime, the previous equation can also be written as

$$
I_{\text {chem }}(x)=\frac{n_{g} V_{r}}{A_{1 \mathrm{P}} f_{J=1}} \sigma_{\mathrm{R}} \cdot I_{3 \mathrm{P}}(x)
$$

Thus, a direct plot of $I_{\text {chem }}(x)$ versus $I_{3 \mathrm{P}}(x)$ should give a linear dependence from which the total reaction cross-section can be obtained, provided that the rest of the parameters are known. The reaction cross-section can even be determined in absolute value owing to the fact that unknown parameters as, for example, the solid angle of detection is the 
(a)
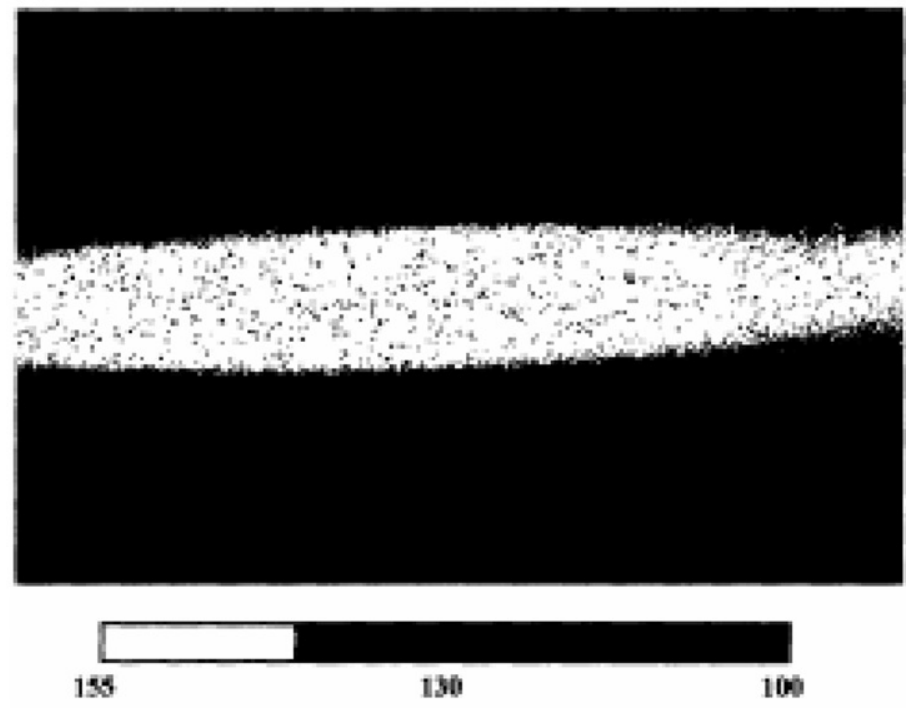

(b)

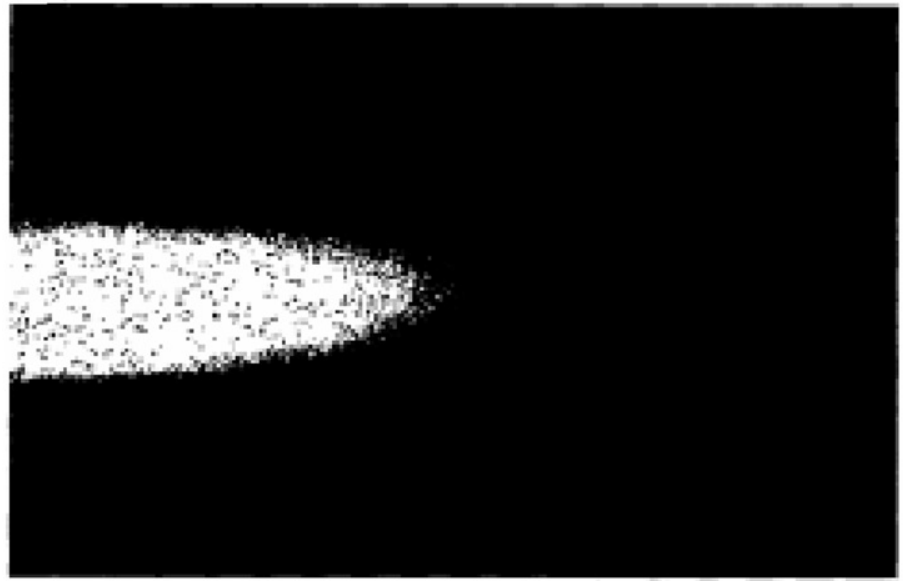

Figure 12. (a) Image of a metastable calcium beam propagating along a vacuum chamber. The spontaneous fluorescence of the forbidden $\mathrm{Ca}\left({ }^{3} \mathrm{P}\right) \rightarrow \mathrm{Ca}\left({ }^{1} \mathrm{~S}_{0}\right)$ transition is displayed in white. (b) Same image but now when a buffer gas is present. Notice the attenuation. This figure is reproduced with permission from [134].

same for $I_{\text {chem }}(x)$ and $I_{3 \mathrm{P}}(x)$ so that it cancels out. Figure 13 displays an example of this linear dependence for the $\mathrm{Ca}\left({ }^{3} \mathrm{P}_{1}\right)+\mathrm{N}_{2} \mathrm{O} \rightarrow \mathrm{CaO} *+\mathrm{N}_{2}$ reaction. The solid line is a numerical fit of the data from which the corresponding reaction cross-section value was deduced. The $\sigma_{\mathrm{R}}$ values obtained are listed in table 1 .

3.2.1 Product reaction distributions by probing the nascent vibro-rotational distribution by laser spectroscopy. Figure 14 shows the experimental set up for the study of alkaline earth plus halogen reaction under beam gas arrangement [85]. 


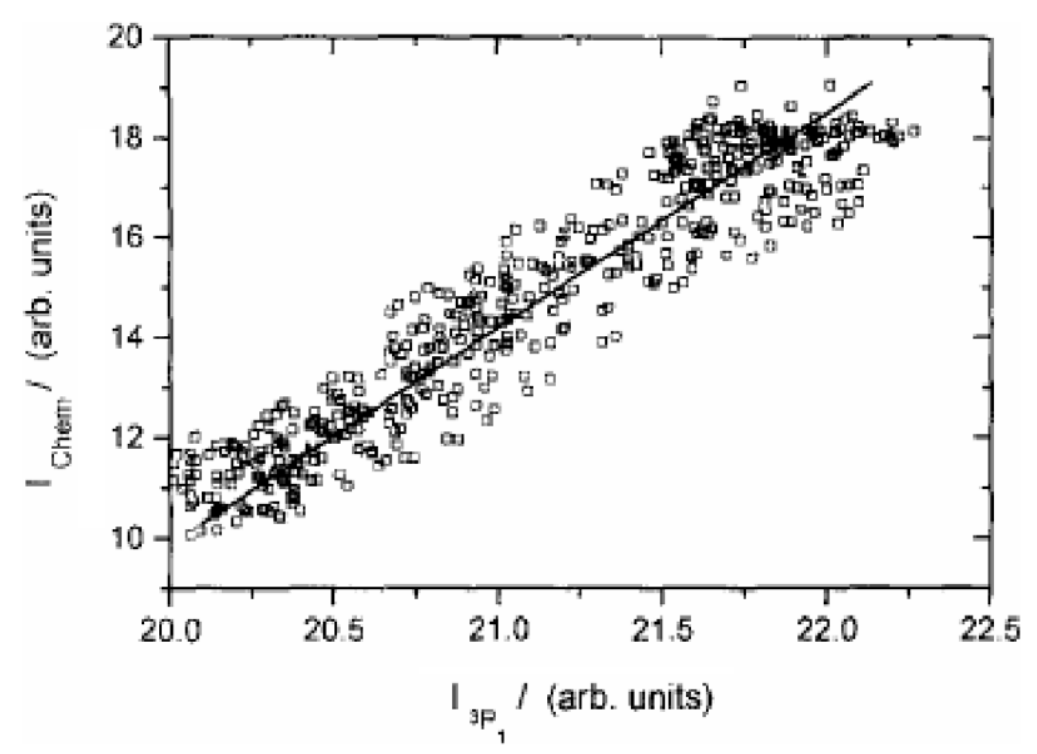

Figure 13. Equation (6) type of representation for the $\mathrm{Ca}\left({ }^{1} \mathrm{P}_{1}\right)+\mathrm{N}_{2} \mathrm{O} \rightarrow \mathrm{CaO} *+\mathrm{N}_{2}$ reaction. A clear linear dependence is noticed from which the reaction cross-section value can be deduced. This figure is reproduced with permission from [134].

Table 1. Reaction cross-sections.

\begin{tabular}{ll}
\hline Reaction & $\sigma_{\mathrm{R}} / \AA^{2}$ \\
\hline $\mathrm{Ca}\left({ }^{3} \mathrm{P}_{\mathrm{J}}\right)+\mathrm{N}_{2} \mathrm{O} \rightarrow \mathrm{CaO} *+\mathrm{N}_{2}$ & $0.13(\mathrm{LBL})$ \\
$\mathrm{Ca}\left({ }^{3} \mathrm{P}_{\mathrm{J}}\right)+\mathrm{CH}_{3} \mathrm{I} \rightarrow \mathrm{CaI}+\mathrm{CH}_{3}$ & $1.3(\mathrm{LBL})$ \\
\hline
\end{tabular}

LBL, results obtained by the Lambert-Beer law.

In this figure, a heated oven is used to generate an effusive magnesium beam which subsequently collides with the $\mathrm{Cl}_{2}$ reactant yielding ground state $\mathrm{MgCl}\left(\mathrm{X}^{2} \Sigma^{+}\right)$that is probed by LIF. Atomic beam fluorescence measurement is used to normalize the LIF spectra with the beam density. Spectra analysis indicates that the nascent diatom is preferentially rotationally excited. In contrast, the $\mathrm{CaCl}$ diatom produced in the $\mathrm{Ca}+\mathrm{Cl}_{2} \rightarrow \mathrm{CaCl}+\mathrm{Cl}$ reaction shows preferred vibrational excitation. This type of reaction, $\mathrm{M}(\mathrm{M}=\mathrm{Mg}$ and $\mathrm{Ca})+\mathrm{X}_{2}(\mathrm{X}=\mathrm{F}, \mathrm{Cl}) \rightarrow \mathrm{MX}+\mathrm{X}$, is known to occur via an electron transfer from the metal atom to the di-halogen whose unstable negative ion, $\mathrm{X}_{2}^{-}$, rapidly dissociates under the Coulombic attraction.

LIF analysis [85] of the nascent $\mathrm{CaCl}$ versus $\mathrm{MgCl}$ product indicates that while the first diatom is formed vibrationally excited, the second is produced rotationally excited. This difference can be explained by the different range over which the electron jumps takes place. While in the Ca reaction this distance occurs at large values so that energy can be allocated in the $\mathrm{Ca}-\mathrm{X}$ coordinate, i.e. in the $\mathrm{CaCl}$ vibrational energy. In the $\mathrm{Mg}$ reaction the electron jump distance is shorter such that there is no possibility for 


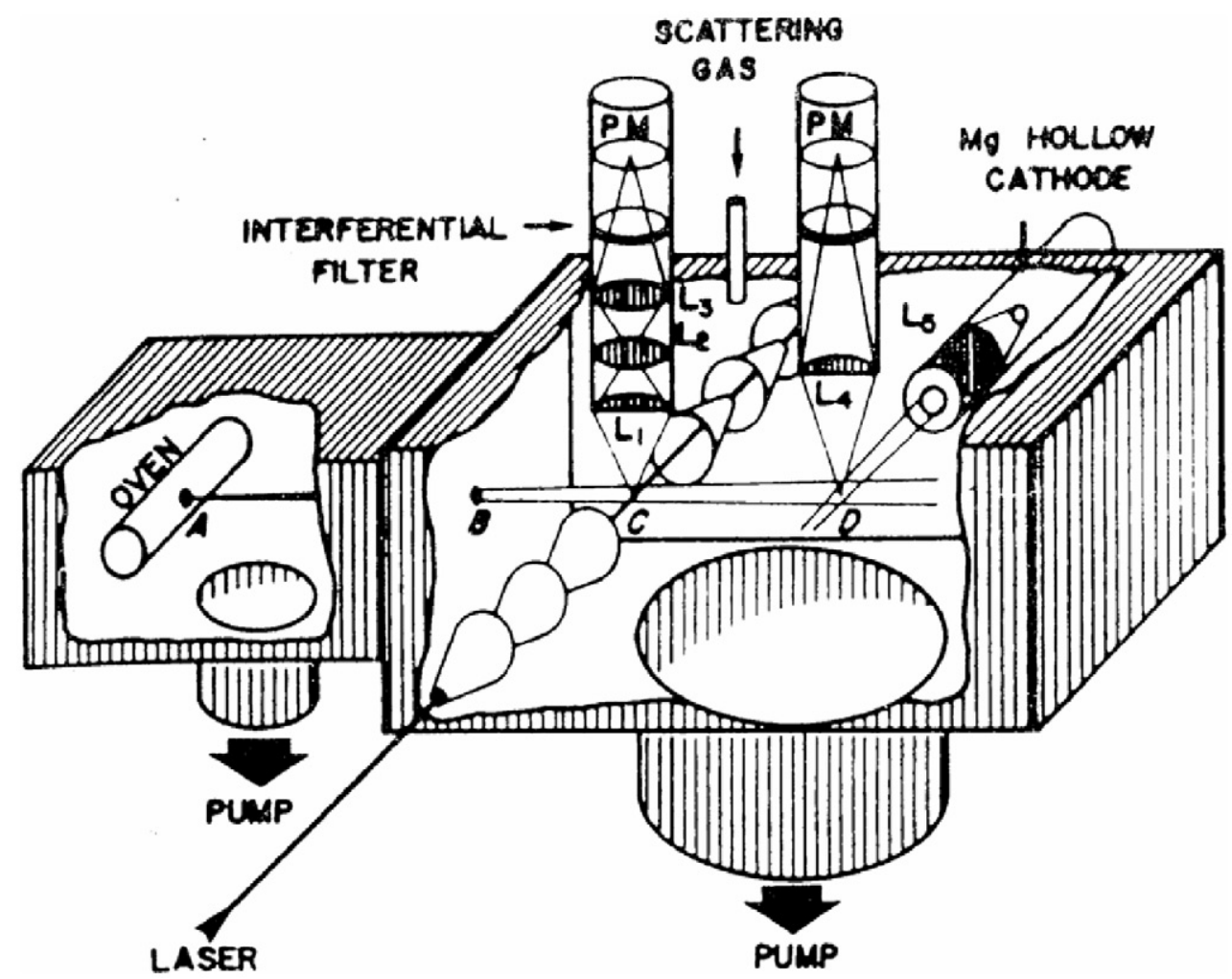

Figure 14. Schematic representation of a beam gas apparatus for alkaline-earth atom reactions. An efussive beam is created by heating an oven containing the metal and then the laser probes the nascent product formed by the beam gas reaction as indicated. This figure is reproduced with permission from [85].

vibrational excitation of the product and most of the energy appears in rotational excitation of the $\mathrm{MgCl}$.

The excited $\mathrm{Sr}\left({ }^{3} \mathrm{P}_{1}\right)+\mathrm{HF} \rightarrow \mathrm{Sr} \mathrm{F}+\mathrm{H}$ reaction was also studied using the pump and probe technique under beam-gas conditions [87, 88]. An argon ion laser pumped a modified Ti:sapphire laser to excite the ground $\mathrm{Sr}$ atoms to the excited $\mathrm{Sr}{ }^{3} \mathrm{P}_{1}$ at $\lambda_{\text {exc }}=689.3 \mathrm{~nm}$. The ground state $\mathrm{SrF}$ product was detected by LIF. To this end either the $\mathrm{B}^{2} \Sigma$ state $(577-587 \mathrm{~nm})$ or the $\mathrm{A}^{2} \Pi_{3 / 2}$ state $(644-653 \mathrm{~nm})$ were excited using a modified tunable linear dye laser.

An example of LIF spectrum of the $\mathrm{SrF}$ formed in the reaction $\mathrm{Sr}\left({ }^{3} \mathrm{P}_{1}\right)+\mathrm{HF} \rightarrow \mathrm{Sr} \mathrm{F}$ $\left(\mathrm{X}^{2} \Sigma, v^{\prime}, J^{\prime}\right)+\mathrm{H}$ is shown in figure $15(\mathrm{a})$. The simulated spectrum is shown in figure 15(b) the comparison between the experimental and simulated LIF distribution allowed to deduce the product vibrational distribution. An example is shown in figure 16 for the $\mathrm{SrF}$ formed in the $\mathrm{Sr}\left({ }^{3} \mathrm{P}_{1}\right)+\mathrm{HF}$. It is of a near Gaussian distribution, i.e. it shows an inverted vibrational distribution.

The analysis of the LIF spectrum allowed to deduce the product energy distribution of the nascent SrF. Such distributions are shown in the form of a triangle plot for the $\mathrm{Sr}+\mathrm{HF}(v=0) \rightarrow \mathrm{Sr} \mathrm{F}+\mathrm{H}$ reaction in figure 17. In these triangular plots the results are 
(a) Wavelength $(\mathrm{nm})$
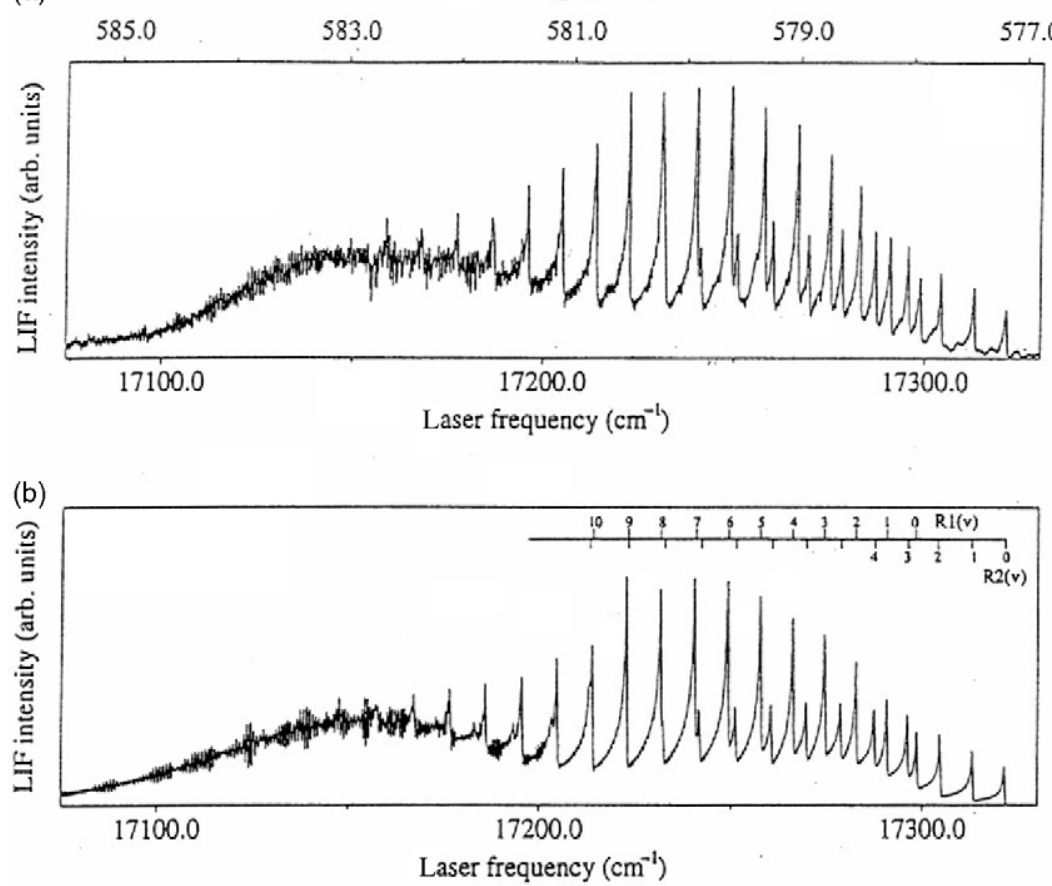

Figure 15. Experimental (a) and simulated (b) spectra of $\operatorname{SrF}$ formed in the reaction of $\operatorname{Sr}\left({ }^{3} \mathrm{P}_{1}\right)$ with HF. The $\mathrm{B}^{2} \Sigma-\mathrm{X}^{2} \Sigma, \Delta v=0$ sequence is shown. LIF, laser-induced fluorescence. This figure is reproduced with permission from [86].

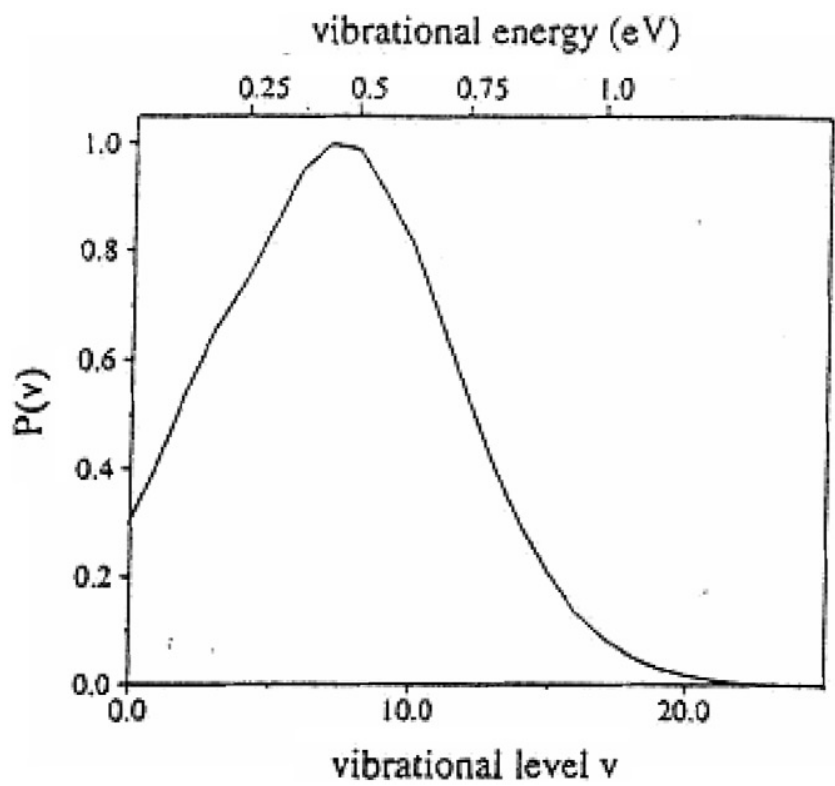

Figure 16. Input vibrational distribution used in the simulated laser-induced fluorescence (LIF) spectrum of the $\mathrm{SrF}$ formed in the $\operatorname{Sr}\left({ }^{3} \mathrm{P}_{1}\right)+\mathrm{HF}$ reaction. This figure is reproduced with permission from [86]. 


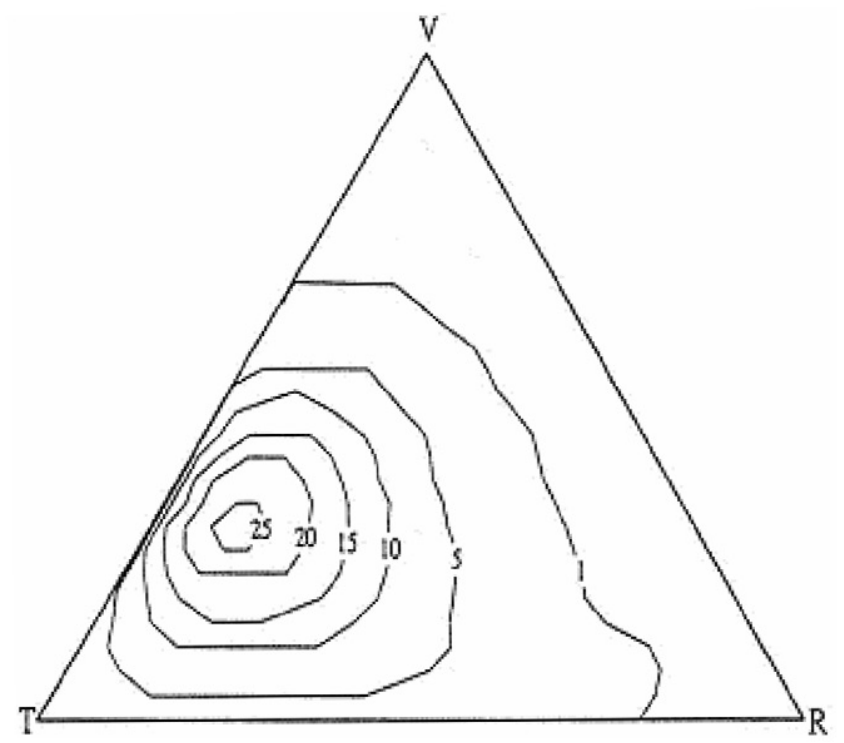

Figure 17. Triangular plot of energy disposal in the $\operatorname{Sr}\left({ }^{3} \mathrm{P}_{1}\right)+\operatorname{HF}(v=0)$ reaction. The experimental results are displayed as contours of relative populations in a triangular coordinate system (see text). The vertices represents the situation where all energy is released into one degree of freedom. This figure is reproduced with permission from [86].

displayed as contours of relative populations. The vertices represent the extreme situations where all energy is disposed into one degree of freedom. Looking at the triangle plot one sees how the highest peak intensity lies closer to the translational and vibrational vertices than to the rotational one.

LIF studies of excited $\mathrm{Sr}$ plus $\mathrm{CH}_{3} \mathrm{~F}, \mathrm{C}_{2} \mathrm{H}_{5} \mathrm{~F}$ and $\mathrm{C}_{2} \mathrm{H}_{4} \mathrm{~F}_{2}$ reactions demonstrate little product vibrational excitation for the $\mathrm{CH}_{3} \mathrm{~F}$ reaction and a moderate increase in the vibrational energy distribution in going to $\mathrm{C}_{2} \mathrm{H}_{3} \mathrm{~F}$ and $\mathrm{C}_{2} \mathrm{H}_{4} \mathrm{~F}_{2}$. In general the small product vibrational excitation cannot be explained in the light of the electron jump mechanism.

The electron transfer mechanism involves an early energy release leading to highly vibrational excited products. The low vibrational excitation deduced from these studies suggests that the reaction energy disposal is dominated by some other mechanism. In the case of $\mathrm{Ca}+\mathrm{CH}_{3} \mathrm{~F}$ a later barrier in the exit channel could explain low product vibration. With respect to the SrF rotational energy it decreases in going from methyl to ethylfluoride as a reactant. This is consistent with a decrease in reaction cross-section as was found in similar reactions [88] probably due to steric effects.

The reactions of laser excited $\mathrm{Sr}\left({ }^{3} \mathrm{P}_{1}\right)$ atoms with various unsaturated flurohydrocarbons, i.e. $\mathrm{CHF}=\mathrm{CH}_{2}, \mathrm{CF}_{2}=\mathrm{CH}_{2}, \mathrm{CHF}=\mathrm{CHF}$, and $\mathrm{C}_{6} \mathrm{H}_{5} \mathrm{~F}$ have been also studied by using LIF. A comparison between the saturated and unsaturated reactants shows that a much lower amount of energy appears as internal energy of the diatom product in reactions with unsaturated compounds. This was rationalized by assuming that unsaturated organic radicals take up more reaction energy than the 


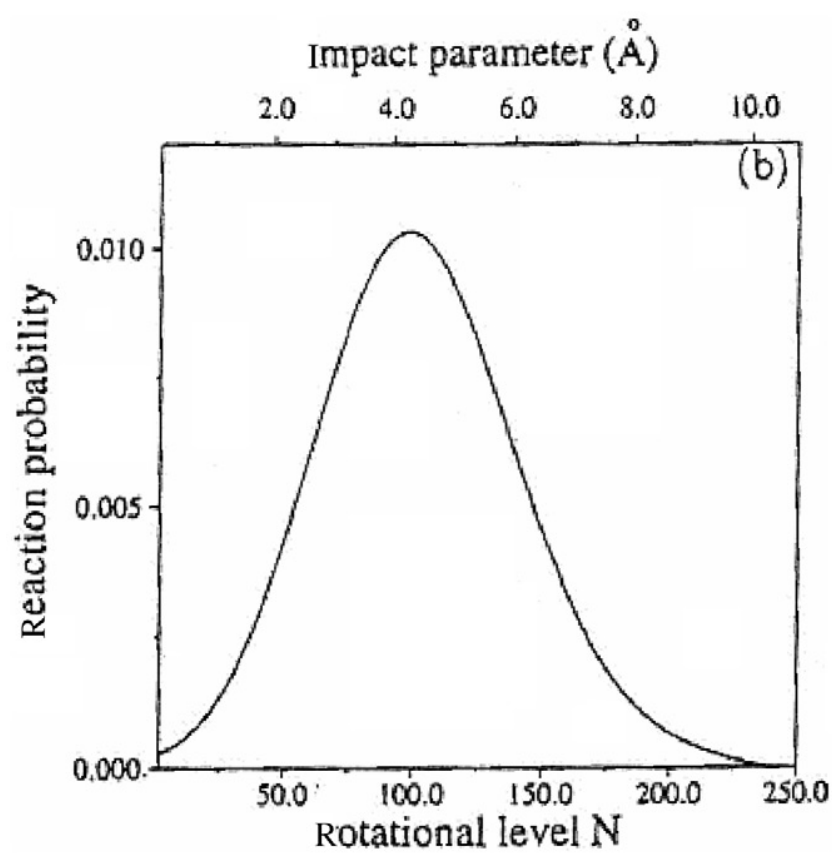

Figure 18. Reaction probability as a function of the rotational $\mathrm{N}$ level for the excited Sr reaction with HF molecules. This figure is reproduced with permission from [86].

saturated radicals. A main conclusion of this study suggested that the presence of a double bond in the organic reactant supports an insertion reaction mechanism.

3.2.2 Product polarization and stereodynamics. The $\mathrm{Sr}+\mathrm{HF}$ reaction can be considered as a $\mathrm{H}+\mathrm{H}^{\prime} \mathrm{L} \rightarrow \mathrm{HH}^{\prime}+\mathrm{L}\left(\mathrm{H}, \mathrm{H}^{\prime}=\right.$ heavy, $\mathrm{L}=$ light $)$ reaction. From the total angular momentum conservation: $\mathbf{J}_{\text {react }}+\mathbf{L}_{\text {react }}=\mathbf{J}_{\text {prod }}+\mathbf{I}_{\text {prod }}$, were $\mathbf{J}$ is the rotational angular momentum and $\mathbf{L}$ is the orbital angular momentum. The latter is given by $|L|=\mu b v$, where $\mu$ is the reduced mass, $b$ is the impact parameter and $v$ is the relative velocity. For the $\mathrm{Sr}+\mathrm{HF}$ the limiting model of $J_{\text {react }} \approx 0$ and $L_{\text {prod }} \approx 0$ was adopted leading to the constraint $J_{\text {prod }} \approx\left|L_{\text {react }}\right| \approx \mu b v_{\mathrm{i}}$. This relation allows the determination of the impact parameter distribution for a specific vibrational level from product rotational distribution, i.e. a certain pair $\left(b, v_{\mathrm{i}}\right)$ leads to one specific $(v, J)$ state.

Figure 18 shows the reaction probability summed over $v$ as a function of the rotational quantum number $N$. The distribution peaks at $b=4.2 \AA$ and impact parameters as high as $9 \AA$ are still contributing to the chemical reaction. The estimated average reaction cross-section at $\left\langle\mathrm{E}_{\mathrm{T}}\right\rangle \approx 0.36 \mathrm{eV}$ was $\sigma_{\mathrm{R}} \approx 70 \AA^{2}$ which is of the same order reported for the $\mathrm{Ca}\left({ }^{3} \mathrm{P}_{\mathrm{J}}\right)+\mathrm{HF}$ reaction for which the reaction cross-section was estimated to be $100 \AA^{2} \leq \sigma_{\mathrm{R}}<170 \AA^{2}$ [89]. The mechanism for the excited $\mathrm{Sr}+\mathrm{HF}$ reaction was suggested to be the electron jump mechanism based on the estimated harpooning radius of $4.1 \AA$, i.e. very close to the peak impact parameter $\approx 4.2 \AA$ and on 
the low ionization potential of $\operatorname{Sr}\left({ }^{3} \mathrm{P}_{1}\right)$. The electron jump mechanism was also involved to explain the observed inverted product vibrational distribution.

Information about the stereodynamics of a chemical reaction can be obtained by measuring polarized chemiluminescence spectra. For a selected molecular transition, the polarization degree, $P$, defined by the expression

$$
P=\frac{I_{\|}-I_{\perp}}{I_{\|}+I_{\perp}}
$$

can be obtained from the direct integration of the measured spectra to have the parallel, $I_{\|}$and perpendicular $I_{\perp}$, emission intensities. Typically for beam-gas reactions the reference axis is taken that of the molecular beam (see below). This magnitude can be more easily calculated for parallel transitions because for them the polarization analysis gives the rotational alignment parameter of the molecule, $\left\langle P_{2}\left(\mathbf{j}^{\prime} \cdot \mathbf{k}\right)\right\rangle$, through simple relations. In the following discussion, $\mathbf{j}^{\prime}, \mathbf{k}$ and $\mathbf{z}$ represent the unit vectors of the rotational product angular momentum, relative velocity and atomic beam velocity and $P_{2}(x)$ the second-order Legendre polynomial.

The degree of polarization of chemiluminescence $P$ can be related to the distribution of angular momentum vectors of the emitting molecule as follows

$$
\begin{gathered}
P(P, R \text { line })=-\frac{3\left\langle P_{2}\left(\mathbf{j}^{\prime} \cdot \mathbf{z}\right)\right\rangle}{4-\left\langle P_{2}\left(\mathbf{j}^{\prime} \cdot \mathbf{z}\right)\right\rangle}, \\
P(Q \text { line })=\frac{3\left\langle P_{2}\left(\mathbf{j}^{\prime} \cdot \mathbf{z}\right)\right\rangle}{2+\left\langle P_{2}\left(\mathbf{j}^{\prime} \cdot \mathbf{z}\right)\right\rangle}
\end{gathered}
$$

where $P$ is given by equation (7).

For a parallel-type transition, only $P$ and $R$ rotational branches are present, and $\left\langle P_{2}\left(\mathbf{j}^{\prime} \cdot \mathbf{z}\right)\right\rangle$ can be calculated by using equation (8). For a perpendicular-type transition, the spectrum may contain either $P, R$ branches or a $Q$ branch and $\left\langle P_{2}\left(\mathbf{j}^{\prime} \cdot \mathbf{z}\right)\right\rangle$ should be calculated by using both equations (8) and (9) for different rotational branches, respectively.

The azimuthal average addition (AAA) theorem [90] is used to correct the beam-gas blurring effect of the rotational alignment given by $\left\langle P_{2}\left(\mathbf{j}^{\prime} \cdot \mathbf{z}\right)\right\rangle$, e.g.

$$
\left\langle P_{2}\left(\mathbf{j}^{\prime} \cdot \mathbf{z}\right)\right\rangle=\left\langle P_{2}\left(\mathbf{j}^{\prime} \cdot \mathbf{k}\right)\right\rangle\left\langle P_{2}\left(\mathbf{k}^{\prime} \cdot \mathbf{z}\right)\right\rangle
$$

To obtain $\left\langle P_{2}(\mathbf{k} \cdot \mathbf{z})\right\rangle$ one follows the procedure described in Prisant et al. [91] whose details are omitted for brevity. This allows us to calculate the rotational alignment referred to the centre-of-mass system. Table 2 contains the alignment parameters for a series of reactions. Note the closeness to the kinematic limit, given by $\left\langle P_{2}\right\rangle=-0.5$, as the system approaches the heavy plus heavy-light limit.

Deviations from the kinematic limit given by the perfect alignment of the product rotational angular momentum can be expected particularly at low collision energies. Such deviations indicate the disposal of angular momentum into product 
Table 2. Alignment parameters $\left\langle P_{2}(\mathbf{j} \cdot \mathbf{k})\right\rangle$ for several reactions.

\begin{tabular}{|c|c|c|c|}
\hline Reaction & $\left\langle P_{2}(\mathbf{j} \cdot \mathbf{k})\right\rangle$ & Method & Ref. \\
\hline $\mathrm{Sr}+\mathrm{CH}_{3} \mathrm{Br} \rightarrow \mathrm{SrBr}+\mathrm{CH}_{3}$ & $-0.34 \pm 0.04$ & LIF & 94 \\
\hline $\mathrm{Sr}+\mathrm{C}_{2} \mathrm{H}_{5} \mathrm{Br} \rightarrow \mathrm{SrBr}+\mathrm{C}_{2} \mathrm{H}_{5}$ & $-0 \cdot 24 \pm 0 \cdot 04$ & LIF & 94 \\
\hline $\mathrm{Sr}+\mathrm{C}_{3} \mathrm{H}_{7} \mathrm{Br} \rightarrow \mathrm{SrBr}+\mathrm{C}_{3} \mathrm{H}_{7}$ & $-0 \cdot 14 \pm 0 \cdot 04$ & LIF & 94 \\
\hline $\mathrm{Ca}\left({ }^{1} \mathrm{D}_{2}\right)+\mathrm{HBr} \rightarrow \mathrm{CaBr}(\mathrm{B})+\mathrm{H}$ & $-0 \cdot 39 \pm 0.012$ & Chem. & 93 \\
\hline $\mathrm{Ca}\left({ }^{1} \mathrm{D}_{2}\right)+\mathrm{HCl} \rightarrow \mathrm{CaCl}(\mathrm{B})+\mathrm{H}\left(E_{\mathrm{T}}=0 \cdot 1 \mathrm{eV}\right)$ & $-0.41 \pm 0.02$ & Chem. & 95 \\
\hline $\mathrm{Ca}\left({ }^{1} \mathrm{D}_{2}\right)+\mathrm{HCl} \rightarrow \mathrm{CaCl}(\mathrm{B})+\mathrm{H}\left(E_{\mathrm{T}}=0 \cdot 17 \mathrm{eV}\right)$ & $-0.44 \pm 0.02$ & Chem. & 91 \\
\hline $\mathrm{Sr}\left({ }^{3} \mathrm{P}_{\mathrm{J}}\right)+\mathrm{CH}_{3} \mathrm{I}$ & $-0 \cdot 48 \pm 0 \cdot 02$ & Chem. & 96 \\
\hline $\mathrm{Sr}\left({ }^{3} \mathrm{P}_{\mathrm{J}}\right)+\mathrm{C}_{2} \mathrm{H}_{5} \mathrm{I}$ & $-0 \cdot 19 \pm 0 \cdot 02$ & Chem. & 96 \\
\hline $\mathrm{Ca}\left({ }^{1} \mathrm{P}_{1}\right)+\mathrm{CH}_{3} \mathrm{I}$ & $-0 \cdot 20 \pm 0.02$ & Chem. & 97,98 \\
\hline $\mathrm{Ca}\left({ }^{1} \mathrm{P}_{1}\right)+\mathrm{C}_{2} \mathrm{H}_{5}$ & $-0.13 \pm 0.02$ & Chem. & 97,98 \\
\hline $\mathrm{Sr}+\mathrm{NOCl}$ & $-0.40 \pm 0.05$ & Chem. & 97,98 \\
\hline $\mathrm{Ca}+\mathrm{NOCl}$ & $-0.35 \pm 0.04$ & Chem. & 99 \\
\hline
\end{tabular}

Chem., chemiluminescence; and LIF, laser-induced fluorescence.

orbital motion. A dynamical model that accounts for these deviations from perfect alignment is constant product orbital angular momentum (CPOAM) [92]. One of the basic assumptions of the CPOAM model is the recognition of a constant product orbital angular momentum, $L^{\prime}$, when the departing atom is very light in comparison with its diatomic partner. One of the main results of such an approach is given by the following expression for the alignment parameter [92].

$$
\left\langle P_{2}\left(\mathbf{j}^{\prime} \cdot \mathbf{z}\right)\right\rangle=Z_{\mathrm{m}}^{2}\left(\frac{1}{3}-\ln Z_{\mathrm{m}}\right)-\frac{1}{2}
$$

where $Z_{\mathrm{m}}=L^{\prime} / L_{\mathrm{m}}$, and $L_{\mathrm{m}}$ is the maximum angular orbital momentum proportional to the average energy available. This model accounts satisfactorily for the product rotational alignment dependence on reagents' translational energy in heavy plus heavy-light systems [93].

\subsection{Crossed-beam studies: translational energy effects}

For neutral-neutral interactions, as far as the collision energy dependence of the reaction cross-section is concerned, three methods have frequently been used in order to vary the relative translational energy of the reagents. They involve the variation of:

(i) the velocity of one or both of the beams;

(ii) the collision angle; and

(iii) the time of flight of at least one reagent.

In the former, the typical implementation consists of changing the relative velocity by varying one beam velocity via the seeding technique. Many applications of this method have been reported and the reader is addressed to a review article [100] for further information. The changing of the collision geometry, i.e. the change of the collision angle, while both beam velocities remain fixed, was first reported by 
Verdasco et al. [101] and nowadays is currently used in crossed-beam experiments for reactive scattering measurements [102]. The philosophy of the time-of-flight (TOF) method is based on the collision of one stationary target (either beam or gas) which collides with a pulsed beam. If reaction takes place, the measurements of the reagent time-of-flight spectrum as well as the product time profile give the time and hence the collision energy dependent reaction cross-section via the modified equation

$$
\sigma_{\mathrm{R}}\left(\frac{E}{t}\right)=\frac{F_{\mathrm{AB}}(t)}{n_{\mathrm{A}}(t) n_{\mathrm{BC}} v_{\mathrm{R}}(t) \Delta V}
$$

in which we have assumed BC as stationary. This method has been used for beam-gas experiments and, not as often, for crossed-beams [103].

A nominal reactive rate coefficient as a function of the average collision energy can be calculated by dividing the chemiluminescence intensity $F_{\mathrm{AB}}(t)$ by the reactant number density $n_{\mathrm{A}}(t)$, e.g. rearranging equation (12) one obtains for the specific rate constant

$$
k\left(\frac{E}{t}\right)=\sigma_{\mathrm{R}}\left(\frac{E}{t}\right) v_{\mathrm{R}}(t)=\frac{F_{\mathrm{AB}}(t)}{n_{\mathrm{BC}} \Delta V n_{\mathrm{A}}(t)}
$$

so that $k(E / t)$ is proportional to $F_{\mathrm{AB}}(t) / n_{\mathrm{A}}(t)$ since $\Delta V$ and $n_{\mathrm{BC}}$ are not time-of-flight dependent quantities.

An example is discussed below for the chemiluminescent $\mathrm{Ca}\left({ }^{1} \mathrm{D}\right)+\mathrm{HBr} \rightarrow \mathrm{CaBr}$ $(\mathrm{A}, \mathrm{B})+\mathrm{H}$ reaction.

Figure 19(a) shows the variation of $\sigma_{\mathrm{A}}(t, E) v$ with collision energy, whereas figure 19(b) gives the excitation function for the electronic channel leading to $\mathrm{B}^{2} \Sigma^{+}$ $\mathrm{CaBr}$, from which it is possible to determine a threshold energy of $20.2 \pm 0.5 \mathrm{~kJ} \mathrm{~mol}^{-1}$.

In figure 19, a series of regularly spaced subsidiary maxima appear at energies above threshold. The width of the oscillations is about $1.5 \mathrm{~kJ} \mathrm{~mol}^{-1}$, comparable with the energy resolution of the beam-beam arrangement.

The origin of the several maxima manifested discerned in the translational excitation function for $\sigma_{\mathrm{B}}(E)$ may be rationalized semi-quantitatively by a model that invokes the two-state Landau-Zener (LZ) model for curve crossing at both $R_{0}$ and $R_{\mathrm{i}}$ together with a simple description of the vibrational dynamics in the reaction transition state. To explain the origin of subsidiary maxima in the excitation function for $\mathrm{B}^{2} \Sigma^{+}$state production, two models for the $\mathrm{Ca}{ }^{1} \mathrm{D}+\mathrm{HBr}$ full collision dynamics were postulated. Common to both is the invocation of a weakly bound, linear transition state [Ca $\cdots$ $\mathrm{Br} \ldots \mathrm{H}]^{* \pm}$ through which all $\mathrm{Ca}{ }^{1} \mathrm{D}+\mathrm{HBr}$ trajectories pass to reach $\mathrm{CaBr}\left(\mathrm{B}^{2} \Sigma^{+}\right)+\mathrm{H}$ products; their difference lies in the way in which excess vibrational energy is channelled in $[\mathrm{Ca} \cdots \mathrm{Br} \ldots \mathrm{H}]^{*^{ \pm}}$to bring about reaction. The existence of a bound, electronically excited van der Waals complex is supported by both theoretical calculations and laser spectroscopy [104].

It is assumed that the predominant initial orbital interaction corresponds to side-on $\mathrm{d} \pi-\sigma^{*}$ overlap on the repulsive $\Pi$ potential. In order to reach $\mathrm{CaBr} \mathrm{B}{ }^{2} \Sigma^{+}$product from $\mathrm{Ca}{ }^{1} \mathrm{D}+\mathrm{HBr}$ starting materials, it was postulated that bending vibrations of $[\mathrm{Ca}-\mathrm{HBr}]^{*}$ 


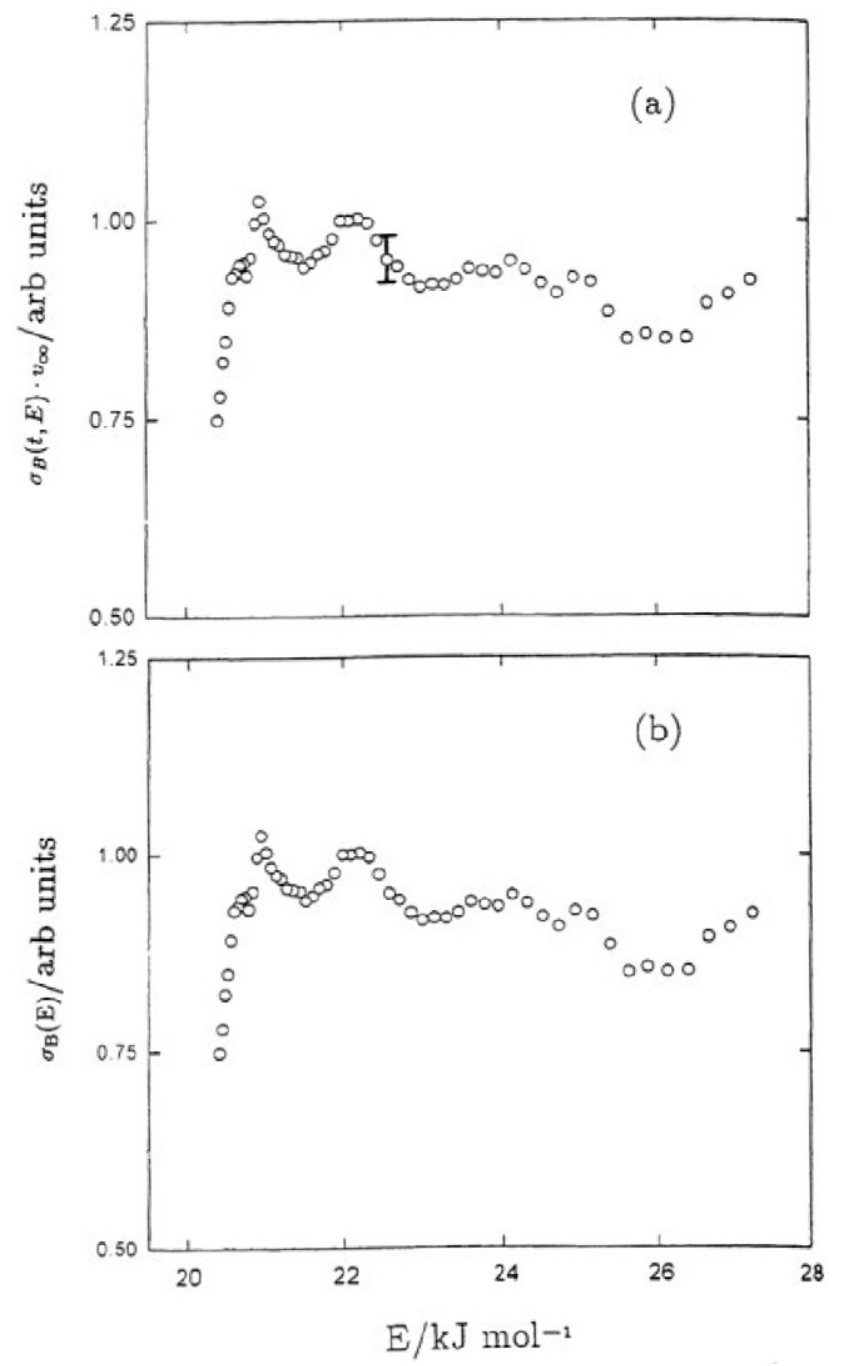

Figure 19. Excitation function for the $\mathrm{Ca}\left({ }^{1} \mathrm{D}\right)+\mathrm{HBr} \rightarrow \mathrm{CaBr}\left(\mathrm{B}^{2} \Sigma^{+}\right)+\mathrm{H}$ reaction. Notice the oscillations in $\sigma_{\mathrm{B}}(t, E) v_{\infty}$ data at energies above $\approx 21.7 \mathrm{~kJ} \mathrm{~mol}^{-1}$. The uncertainty associated with measurements is indicated by the magnitude of the representative error bar. This figure is reproduced with permission from [104].

enable the initially non-linear complex to attain the liner transition state configuration required for subsequent break-up to form $\mathrm{CaBr} \mathrm{B}^{2} \Sigma^{+}$over a potential of $\Sigma$ symmetry with conservation of electronic orbital angular momentum in the exit channel. The spacing between maxima in the plot of $\sigma_{\mathrm{B}}(E)$ versus energy may then reflect the bending vibrational mode of $[\mathrm{Ca}-\mathrm{HBr}]^{*}$ at these energies. For non-collinear geometries in the entrance channel, it can be postulated that the reactants approach each other over an $\mathrm{A}^{\prime}$ state potential and that the observed vibrational dynamics of $[\mathrm{Ca}-\mathrm{HBr}]^{*}$ reflect essentially free rotation of $\mathrm{Ca}-\mathrm{HBr}$ to form $\mathrm{Ca}-\mathrm{BrH}$ within the complex prior to electron harpooning. 


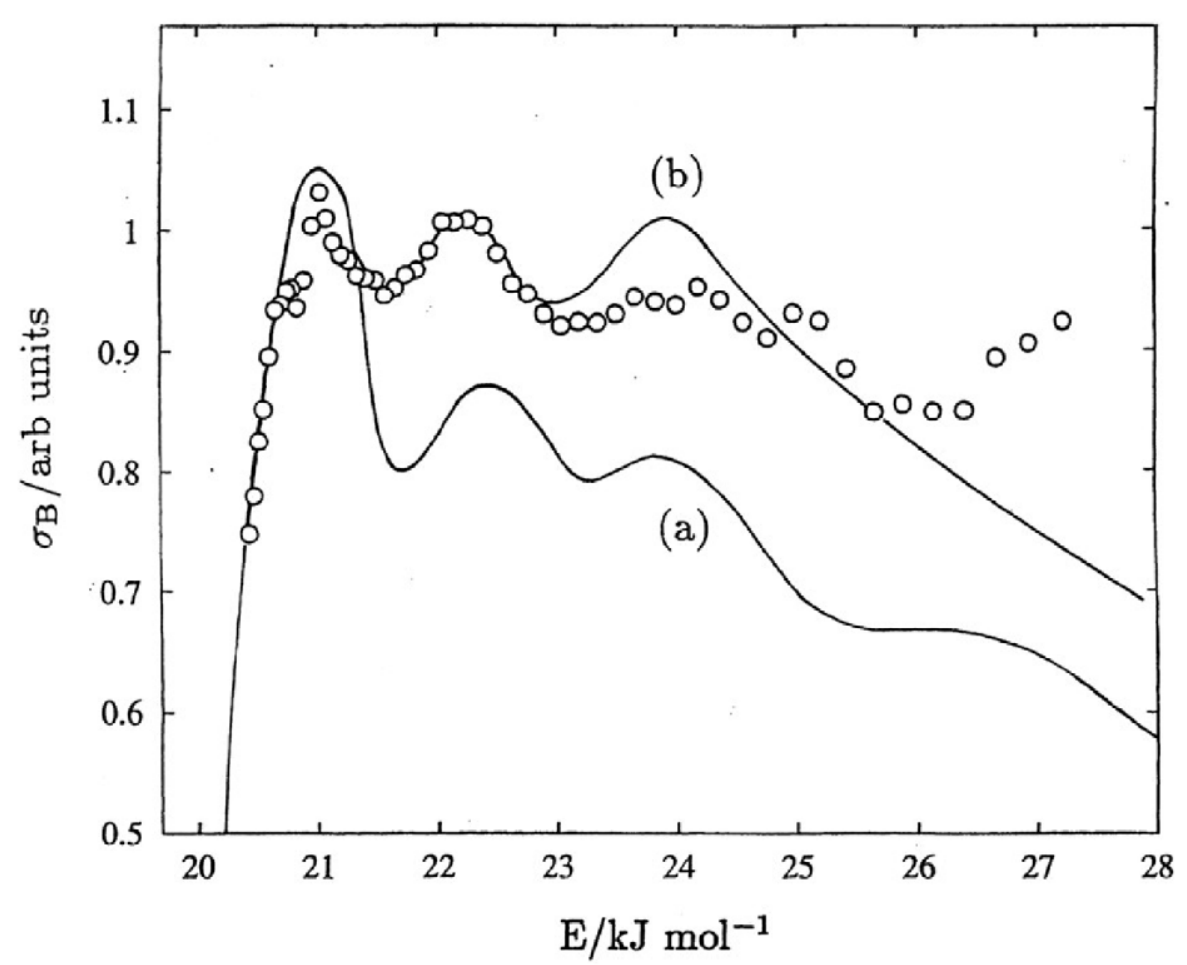

Figure 20. Comparison of predicted and experimental excitation functions: solid lines, (a) energetically democratic and (b) vibrationally selective models; open for the $\mathrm{Ca}\left({ }^{1} \mathrm{D}\right)+\mathrm{HBr} \rightarrow \mathrm{CaBr}\left(\mathrm{B}^{2} \Sigma^{+}\right)+\mathrm{H}$ reaction circles, data of figure 19. This figure is reproduced with permission from [104].

The first mechanism proposed here for the $[\mathrm{Ca}-\mathrm{HBr}]^{*} \rightarrow \mathrm{CaBr} \mathrm{B}^{2} \Sigma^{+}+\mathrm{H}$ vibrational dynamics is a statistical 'democratic' model in which a reactive trajectory can 'elect' to pass through the $v=0,1$ or 2 , etc. vibrational channels of [Ca $\mathrm{HBr}]^{* \pm}$, depending upon which channels are energetically accessible. The result is displayed as curve (a) in figure 20. The four lowest vibrational levels of [Ca $\mathrm{HBr}]^{*}$ are included in the model with a constant energy spacing $\Delta E$ of $184 \mathrm{~cm}^{-1}\left(2.2 \mathrm{~kJ} \mathrm{~mol}^{-1}\right)$. It is clear from inspection that the statistical democratic model fails to account qualitatively for the observed variation of $\sigma_{\mathrm{B}}(E)$ with $E$ at energies above the $v=0$ threshold.

The second model represents the opposite in terms of vibrational energy consumption, in that reactive trajectories are considered to pass exclusively through the highest lying vibrational channel that is available at a given energy, with a concomitant reduction in the translational energy requirement for curve crossing at $R_{0}$ and surmounting the adiabatic barrier at $R_{\mathrm{i}}$. A constant spacing of $184 \mathrm{~cm}^{-1}$ between the lowest four bending vibrational levels of [Ca-HBr] $]^{*}$ is again assumed except for $\Delta E(v=1$ to $v=0)$, for which $167 \mathrm{~cm}^{-1}$ generates a better fit to the data. Curve (b) of figure 20 indicates that this model fits the experimental result with reasonable qualitative accuracy for energies up to that of the $[\mathrm{Ca} \mathrm{HBr}]^{*} v=3$ level. That the excitation function for $\mathrm{CaBr} \mathrm{A}^{2} \Pi$ formation from $\mathrm{Ca}{ }^{1} \mathrm{D}+\mathrm{HBr}$ reagents does not exhibit subsidiary maxima may be due to one or more factors. The most important is that side-on overlap of the $\mathrm{d} \pi \mathrm{Ca}{ }^{1} \mathrm{D}$ orbital with 
$\mathrm{HBr} \sigma^{*}$ does not require a vibrationally mediated change in electronic state in the $[\mathrm{Ca} \cdot \cdots \mathrm{HBr}]^{*}$ transition state to proceed to $\mathrm{CaBr} \mathrm{A}^{2} \Pi$ with conservation of electronic orbital angular momentum; in this case, the reactive evolution in the entrance channel can proceed entirely over the repulsive potential of $\Pi$ symmetry, consistent with a smoothly declining excitation function beyond threshold. Additional factors are described in Garay et al. [105]. Other types of modifications have been incorporated in the standard Landau-Zener treatment. An example of it is discussed below for the $\mathrm{Ca}^{*}+\mathrm{HCl} \rightarrow \mathrm{CaCl}^{*}+\mathrm{H}$ reaction.

Figure 21 shows the collision energy dependence of the cross-section for the $\mathrm{Ca} *\left({ }^{1} \mathrm{D}_{2}\right)+\mathrm{HCl} \rightarrow \mathrm{CaCl}^{*}\left(\mathrm{~A}^{2} \Pi\right)+\mathrm{H}$ chemiluminescent reaction. The open symbols represent experimental data measured under high-resolution cross-beam conditions and the solid line is a modified time-dependent Landan-Zener (LZ) treatment [106] which seems to account satisfactorily for the observed transactional features of the excitation function such as, for example, a steep rise at low energy, a pronounced maximum followed by a sharp decrease at higher energy. As illustrated in the lower panel of figure 22 the typical Landau-Zener model does not reproduce the observed collisional energy dependence of the reaction cross-section.

The standard Landau-Zener treatment describes non-adiabatic processes as occurring almost instantaneously in relation to the time of passage through the crossing region, i.e. the position of the crossing between the entrance (neutral) and exit (ionic) potential energy surface. However these chemiluminescent reactions require a rearrangement of the intermediate charge-transfer molecular complex (see bellows) and consequently, their time scales can be much longer than those typical of atomic processes. The modified Landau-Zener treatment which described successfully the collision energy dependence of the $\mathrm{Ca}^{*}+\mathrm{HCl} \rightarrow \mathrm{CaCl}^{*}+\mathrm{H}$ reaction is based on two main requirements:

(i) the reagents' interaction in specific geometries, particularly taking into account the anisotropy due to the $\mathrm{Ca}^{*}$ orbital alignment and to the $\mathrm{HX}$ molecular orientation; and

(ii) a necessary synchronization between times for the non-adiabatic transition and for triggering the rearrangement of the intermediate collision complex.

The latter condition imposes some restriction on the range of impact parameters which can lead to reaction. Basically a lower limit is obtained by the requirement that the time required for a transition must be comparable to, or longer than, the time associated with molecular rearrangement. With regards to the first requirement, an accurate picture of the neutral surfaces, including the characterization of their crossing with the ionic surfaces, requires knowledge of three anisotropic terms:

(i) the van der Waals component;

(ii) the electrostatic component; and

(iii) the charge transfer effects.

Taking into account these three contributions, the main approaching geometries for the Ca* $\left({ }^{1} \mathrm{P}_{1}\right)+\mathrm{HX}$ entrance channels have been evaluated and are shown in figure 22 . In the top panel the schematic representation of the atomic $4 \mathrm{~s} 4 \mathrm{p} \mathrm{Ca}$ and $\mathrm{XH}$ molecule is shown, in the lower panel the schematic representation of the main approaches between 


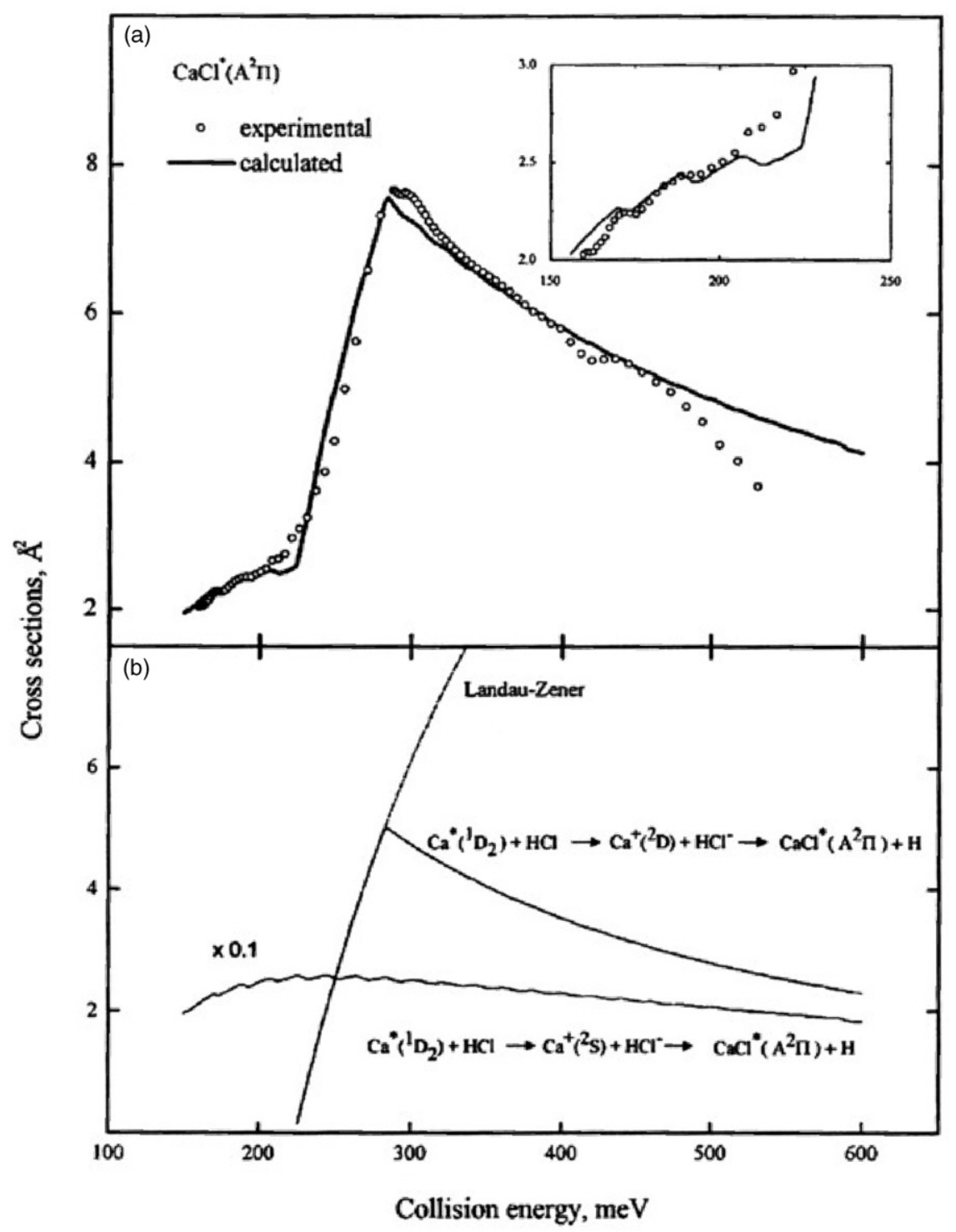

Figure 21. (a) Collision energy dependence of the cross-section for the $\mathrm{Ca}^{*}\left({ }^{1} \mathrm{D}_{2}\right)+\mathrm{HCl} \rightarrow \mathrm{CaCl} *\left(A^{2} \Pi\right)+\mathrm{H}$ chemiluminescent reaction. Open symbols are normalized experimental data and the solid line is obtained combining the calculated effect of two different reaction paths, using the dynamical treatment illustrated in the text. The blow-up emphasizes stair-like behaviours observed at low collision energy. (b) Individual contributions calculated for each reaction path with a rearrangement time of $10 \mathrm{fs}$ for all channels. The typical Landau-Zener behaviour, with no time restriction, is also shown for comparison. This figure is reproduced with permission from [106].

the $\mathrm{Ca}\left({ }^{1} \mathrm{P}_{1}\right)$ and $\mathrm{HX}$ molecule is illustrated. The most favourable geometries are framed. They correspond to figures 22(a), (b) and (f) and represent the most favourable geometries, given the intermediate collision complex geometries in figures 22(d) and (f) also correlate with $\mathrm{Ca}^{*}\left({ }^{1} \mathrm{D}_{2}\right)+\mathrm{HX}$ entrance channels. 
Linear

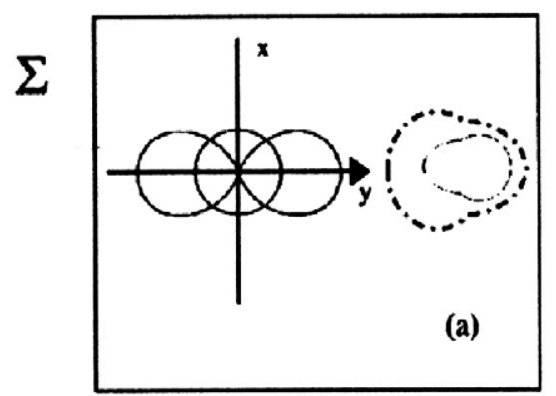

$\Pi$

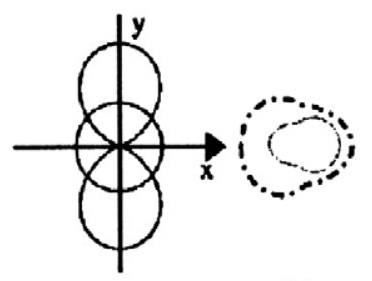

(c)
Nonlinear

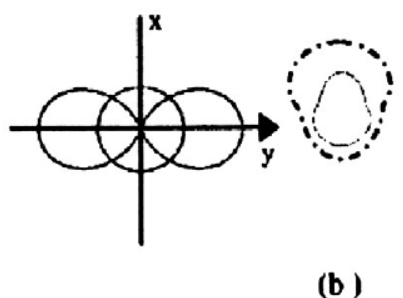

(b)

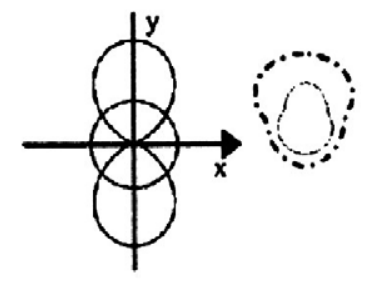

(d)
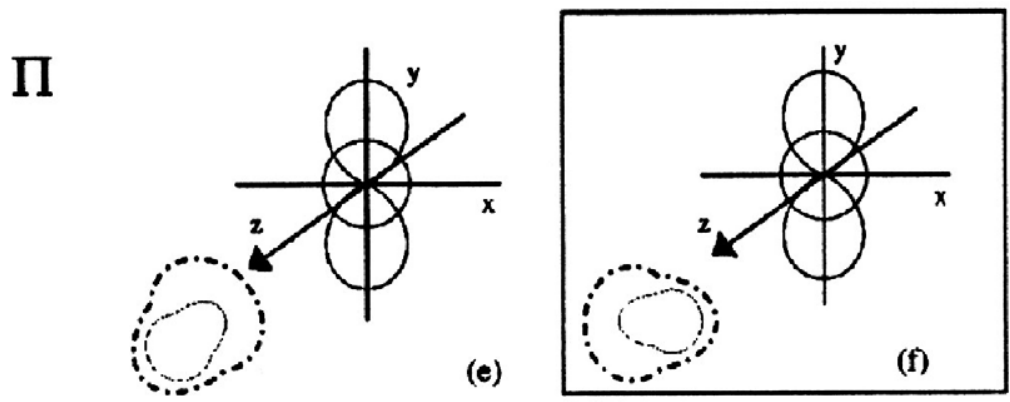

$\mathrm{A}^{\prime \prime}$

Figure 22. Schematic representation of main approaches of reactants $\mathrm{Ca} *\left({ }^{1} \mathrm{P}\right)$ and $\mathrm{HX}$ : most favourable geometries are framed. This figure is reproduced with permission from [106].

Aquilanti and coworkers [106] have estimated these potential energy surfaces for the $\mathrm{Ca}^{*}\left({ }^{1} \mathrm{D}_{2}\right)+\mathrm{HX} \quad(\mathrm{X}=\mathrm{Cl}, \mathrm{Br}) \rightarrow \mathrm{CaX}^{*}+\mathrm{H}$ reaction and an example of cuts of the potential energy surfaces is shown in figure 23 for the $\mathrm{Ca}^{*}\left({ }^{1} \mathrm{D}_{2}\right)+\mathrm{HCl}$ case. Here the outer potential energy crossing in the entrance neutral channel (labelled 1 in the figure) is responsible for the opening of the path to the inner one (labelled 2), which can lead to reaction products $\mathrm{CaX}$ in the two electronic excited states $\mathrm{A}^{2} \Pi$ and $\mathrm{B}^{2} \Sigma$, observed in the chemiluminescent reaction.

Mixing between states associated to the atomic ${ }^{1} \mathrm{D}_{2}$ and ${ }^{1} \mathrm{P}_{1}$ is produced by the outer crossing. On the other hand, the inner one occurs between the entrance channel $\mathrm{Ca}^{*}$ 


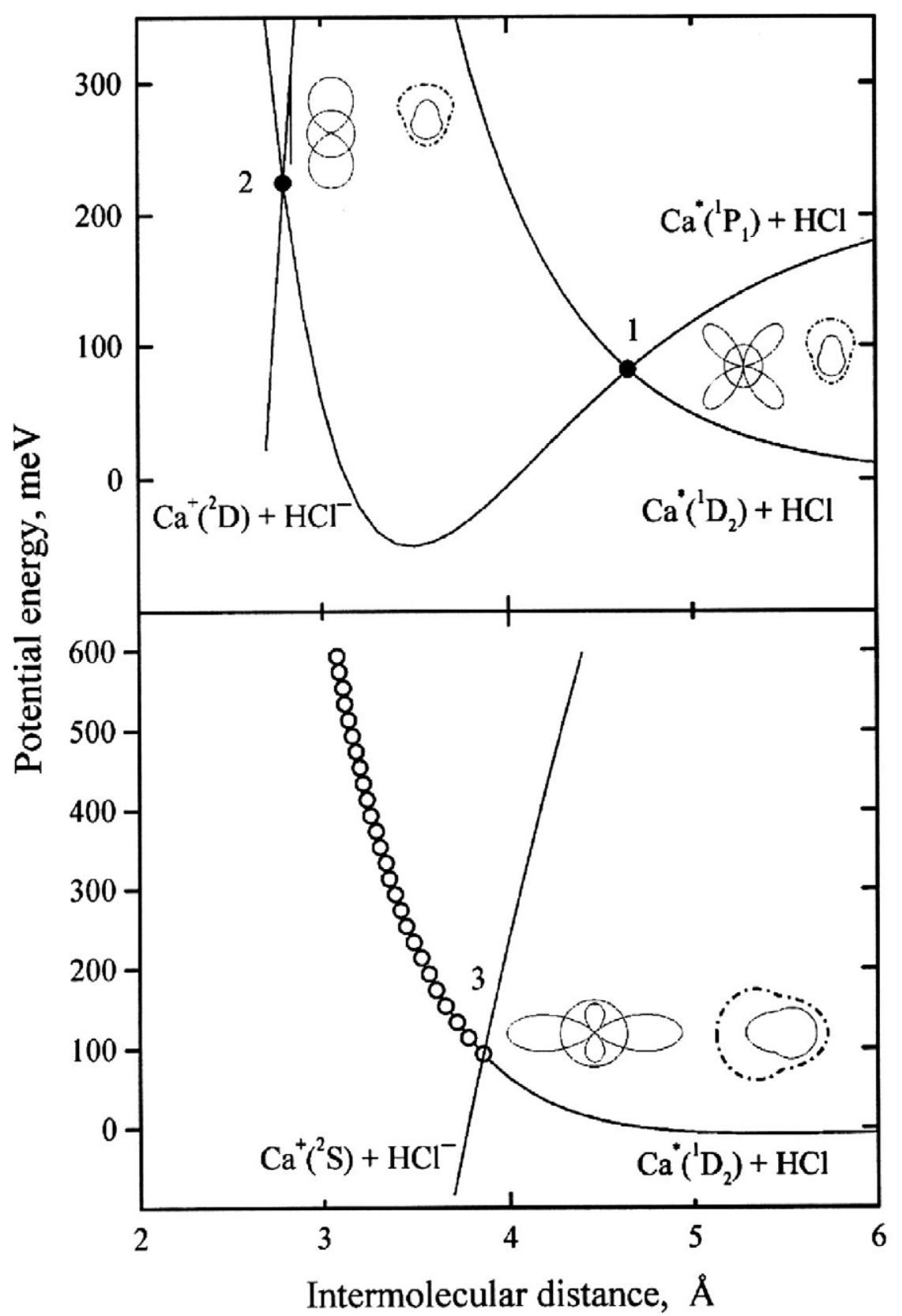

Figure 23. Cuts of the potential energy surfaces in the entrance channels of the $\mathrm{Ca}^{*}-\mathrm{HBr}$ reaction. The upper panel represents the interaction for (d) and (f), illustrated in figure 22, leading to excited $\mathrm{CaBr}^{*}$ product. The pictorial view, which describes the mixing of ${ }^{1} \mathrm{D}$ and ${ }^{1} \mathrm{P}$ atomic states favouring the $4 s$ transfer (circles), refers to (d) [(f) behaves similarly]. The lower panel refers to the case of a collinear approach, with both the atomic $3 \mathrm{~d}$ orbital and the $\mathrm{HBr}$ bond aligned along the intermolecular axis (the pictorial view corresponds to figure 22(a)). This configuration contributes to the formation of the products in both the ground and excited electronic states. Ionic curves are also shown and the potentials are parameterized as discussed. This figure is reproduced with permission from [106]. 


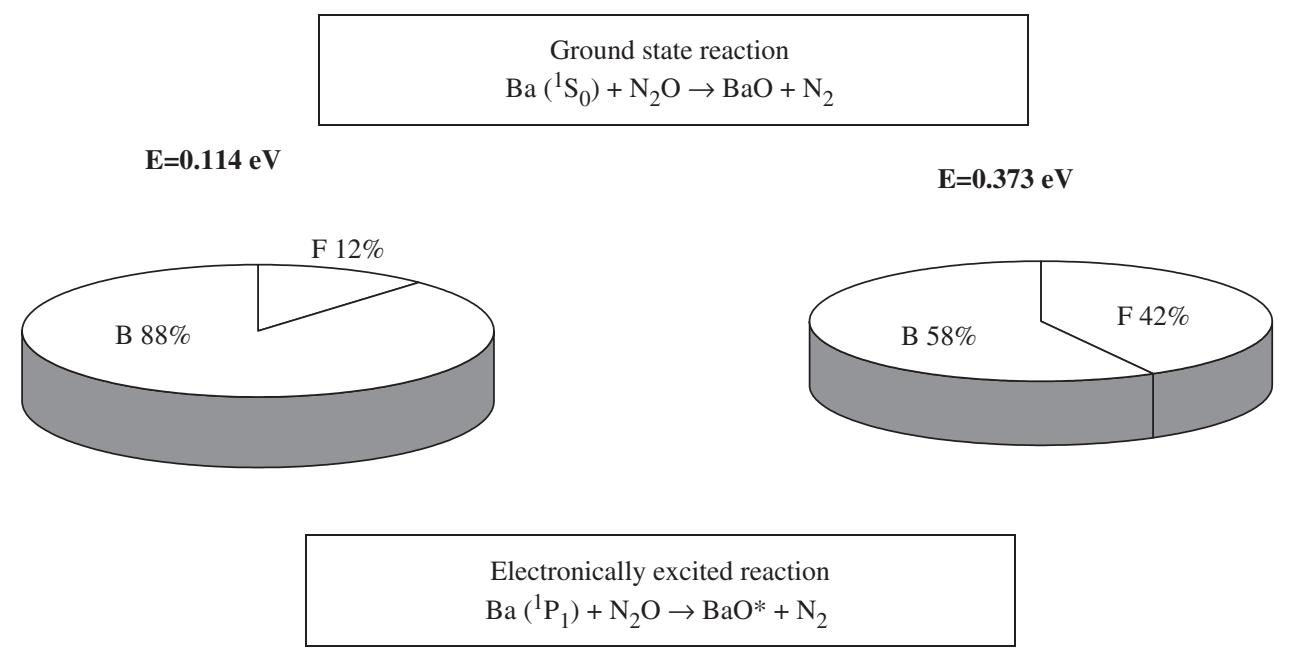

Figure 24. Collision effects of ground state reaction $\mathrm{Ba}\left({ }^{1} \mathrm{~S}_{0}\right)+\mathrm{N}_{2} \mathrm{O} \rightarrow \mathrm{BaO}+\mathrm{N}_{2}$ and electronically excited reaction $\mathrm{Ba}\left({ }^{1} \mathrm{P}_{1}\right)+\mathrm{N}_{2} \mathrm{O} \rightarrow \mathrm{BaO}^{*}+\mathrm{N}_{2}$.

$\left({ }^{1} \mathrm{P}_{1}\right)+\mathrm{HCl}$ and the ionic potential. This crossing involves an electron jump from the $4 \mathrm{~s}$ atomic orbital of $\mathrm{Ca}^{*}$ to the lowest unoccupied antibonding molecular orbital (LUMO) of $\mathrm{HCl}$. As shown in the figure and discussed above this electron transfer preferentially occurs when the $\mathrm{Ca}^{*} 4 \mathrm{p}$ orbital and the HX bond both are perpendicularly aligned with respect to the intermolecular axis. Finally, the curve crossing depicted in lower panel of figure 23 displays the transfer of the $3 \mathrm{~d}$ electron. It allows the chemical reaction in the collinear approach which leads to the formation of $\mathrm{CaCl}$ in the electronic ground state $\mathrm{X}^{2} \Sigma$.

3.3.1 Dynamical and stereodynamical effects in excited reactions. Electronic excitation can also change the shape and symmetry of the potential energy surface and it may induce a distinct reaction mechanism compared to that of the ground state reaction. This seems to be the case with the $\mathrm{Zn}+\mathrm{H}_{2}$ reaction. The ground state reaction, i.e. $\mathrm{Zn}$ $\left({ }^{1} \mathrm{~S}\right)+\mathrm{H}_{2}$, occurs via an abstraction mechanism involving the $\mathrm{Zn} \cdot \cdots \mathrm{H} \cdot \cdots \mathrm{H}$ intermediate that leads into preferential $\mathrm{ZnH}$ vibrational excitation. In contrast, the excited $\mathrm{Zn}\left(4 \mathrm{p}{ }^{3} \mathrm{P},{ }^{1} \mathrm{P}\right)+\mathrm{H}_{2}$ reactions are dominated by an insertion mechanism, which implies the formation of a very short-lived bent $\mathrm{H}-\mathrm{Zn}-\mathrm{H}$ intermediate. The latter subsequently dissociates into $\mathrm{ZnH}+\mathrm{H}$ with little vibrational excitation but with high rotational excitation.

Another example of mechanism change upon electronic excitation of the reagents is also that of $\mathrm{Ba}\left({ }^{1} \mathrm{~S},{ }^{1} \mathrm{P}\right)+\mathrm{N}_{2} \mathrm{O} \rightarrow \mathrm{BaO}+\mathrm{N}_{2}$ reactions which were studied under crossbeam conditions [107]. Figure 24 depicts in a pictorial manner the distinct collision effects of these two reactions. While for the ground state reaction $\mathrm{Ba}\left({ }^{1} \mathrm{~S}_{0}\right)$ the forward scattering of the $\mathrm{BaO}$ product increases more that a factor of three, as the collision energy varies from $0.114 \mathrm{eV}$ to $0.373 \mathrm{eV}$, the overall angular distribution remains 


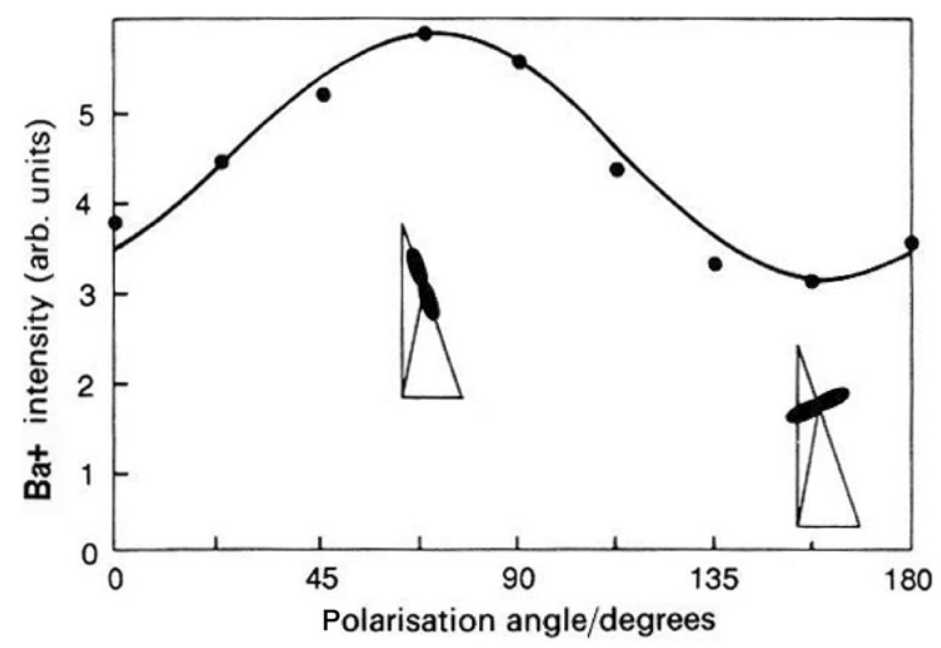

Figure 25. Polarization dependence of $\mathrm{Ba}^{+}$intensity produced in the $\mathrm{Ba}+\mathrm{Cl}_{2} \rightarrow \mathrm{Ba}^{+}+\mathrm{Cl}_{2}^{-}$reaction. The peak corresponds to the $\mathrm{p}$ orbital aligned along the relative velocity vector. This figure is reproduced with permission from [109].

constant for the $\mathrm{Ba}\left({ }^{1} \mathrm{P}\right)$ excited reaction product upon a similar increase of the collision energy.

This difference between the ground and the excited reaction reflects the predominance of collinear attack, responsible of the strong backward character, for the excited one. The excited reaction shows predominance for high impact reactive trajectories, i.e. near T-shape, compared to ground reaction.

The reactions of alkaline-earth metal atoms with hydrogen halide molecules, $\mathrm{M}(\mathrm{Be}, \mathrm{Mg}, \mathrm{Ca}, \mathrm{Sr}, \mathrm{Ba})+\mathrm{HX}(\mathrm{X}=\mathrm{F}, \mathrm{Cl}, \mathrm{Br}, \mathrm{I}) \rightarrow \mathrm{MX}+\mathrm{H}$, have been studied extensively since the early days of reaction dynamics. As mentioned in the introduction the reactivity within this reaction family exhibits a particularly interesting behaviour. Because of the $n s^{2}$ valence-shell electronic structure of the alkaline-earth atoms. Two possibilities appear for the detailed reaction mechanism: direct halogen abstraction via a harpoon mechanism, and an insertion pathway in which the $\mathrm{H}-\mathrm{M}-\mathrm{X}$ potential well is sampled. In some reactions of this family (e.g. $\mathrm{Ca}+\mathrm{HF}$ ) reaction, the insertion pathway has been shown to be dominant, whereas in others, (e.g. $\mathrm{Ba}+\mathrm{HBr}$ and $\mathrm{Ba}+\mathrm{HCl}$ ), the reactions appear to proceed through a direct abstraction pathway.

As for collision energy effects, a remarkable collision energy dependence was found in the $\mathrm{Ba}+\mathrm{IH} \rightarrow \mathrm{BaI}+\mathrm{H}$ reaction dynamics investigated by Zare and coworkers [108] under crossed-beam and beam-gas conditions over the 3-17 $\mathrm{kcal} \mathrm{mol}^{-1}$ range. Between low and high collision energies, a transition occurs in the intensity, width, and peak location of the product vibrational and rotational population distributions. This transition is estimated to occur at approximately $5 \mathrm{kcal} \mathrm{mol}^{-1}$. While for collision energies smaller than $5 \mathrm{kcal} \mathrm{mol}^{-1}$, the product vibrational distribution is bell shaped and peaks at $v=12$ for collision energies larger than $5 \mathrm{kcal} \mathrm{mol}^{-1}$, a second maximum appears at $v=0$ in the vibrational distribution. 
The impact parameter, $b$, is related to orbital angular momentum, $L$, through the expression

$$
|L|=\mu\left|v_{\text {rel }}\right| b,
$$

where $\mu$ and $v_{\text {rel }}$ are the reduced mass and the relative velocity of the colliding particles. In the reaction

$$
\mathrm{Ba}+\mathrm{HI} \rightarrow \mathrm{BaI}+\mathrm{H}
$$

the rotational angular momentum of the reagent $\mathrm{HI}$ is small compared to that found in the product BaI. The orbital angular momentum of the products is relatively small, and can also be neglected. Since in an isolated system total angular momentum is an invariant, conservation of angular momentum gives:

$$
\mu \cdot\left|v_{\mathrm{rel}}\right|_{b}=\left|J_{\mathrm{BaI}}\right|
$$

where $J_{\mathrm{BaI}}$ is the rotational angular momentum of $\mathrm{BaI}$, and its measure is given by

$$
\left|J_{\mathrm{BaI}}\right|=\tilde{n} \sqrt{J(J+1)}
$$

This equation provides a direct link between spectroscopically observable product internal states and impact parameter distribution leading to reactive collisions. Since the impact parameters are not controlled, i.e. the reaction occurs from a range of $b$ values, each weighted by $2 \pi b$, the rotational product distribution for a given vibrational level reflect the specific opacity functions, that is, the reaction probability as a function of the impact parameter. In conclusion for these heavy+heavy-light kinematically constrained reactions the distributions of impact parameters leading to reaction can be deduced by measuring the product rotational state distribution.

As far as rotational energy product disposal is concerned, it was found that as the collision energy is increased above $5 \mathrm{kcal} \mathrm{mol}^{-1}$, the BaI rotational excitation is very near the energetic limit, and the maximum for the $\mathrm{BaI}(v=0)$ rotational population distribution changes from $J=415.5$ to $J=538.5$. However, below $5 \mathrm{kcal} \mathrm{mol}^{-1}$, the maximum remains unchanged around $J=420.5$. Detailed analysis of the collision energy dependence of the specific opacity functions allows clarifying the role of conservation of energy and angular momentum in this reaction dynamics. Whereas at low collision energies, the maximum reactive impact parameter, $b_{\max }$, is determined by an angular momentum (centrifugal) barrier, at $r$ collision energies larger than $5 \mathrm{kcal} \mathrm{mol}^{-1}$, the conservation of energy determines the value of $b_{\max }$. These two processes were therefore identified as the mechanisms that control the $\mathrm{Ba}+\mathrm{HI}$ reaction cross-section and are responsible for the bimodal character of the BaI product internalstate distribution.

Even in ground state reactions there are dynamical consequences of the divalent nature of alkaline-earth atoms. The presence of two valence electrons in alkaline-earth 
atoms may originate some differences in the reaction dynamics if the reaction involves divalent oxygen atoms or molecule containing two halogen atoms. Typical examples of these distinct dynamics are the $\mathrm{Ba}+\mathrm{NO}_{2}$ and the $\mathrm{Ba}+\mathrm{Cl}_{2}$ reactions. In the former the existence of long lived collision intermediates despite very large exothermicities was evidenced by the angular and velocity distributions measured under crossed-beam conditions. The existence of this intermediate is favoured from barriers to transfer of the second electron necessary to form ground state products. Indeed long-range electron transfer produces an ionic complex $\mathrm{Ba}^{+} \mathrm{NO}_{2}^{-}$which does not correlate to ground state $\mathrm{BaO}$ so it may survive many rotational periods, despite highly exoergic product channels available. Formation of ground state $\mathrm{BaO}$, implies a crossing to another electronic surface requiring the transfer of the second electron.

The transfer of the second electron to the LUMO of $\mathrm{NO}_{2}^{-}$is symmetry forbidden. The transfer of this second electron seems to involve excited states resulting in a significant potential barrier. Thus when these complexes dissociate by the transfer of the second electron, the large translational energy release is due to their decay through the exit barrier.

Lee and coworkers [109] studied the dynamics of the $\mathrm{Ba}\left(6 \mathrm{p}{ }^{1} \mathrm{P}\right)+\mathrm{Cl}_{2}$ system which yields both $\mathrm{BaCl}^{+}+\mathrm{Cl}^{-}$reaction products and $\mathrm{Ba}^{+}+\mathrm{Cl}_{2}^{-}$charge-transfer products, the two processes being characterized by their chemi-ionization emission. At $3 \mathrm{eV}$ total energy, laser excitation to $\mathrm{Ba}(6 \mathrm{p})$ enhanced the $\mathrm{Ba}^{+}$intensity measured in the backward direction. As shown in figure 25 , this $\mathrm{Ba}^{+}$intensity is a maximum when the $\mathrm{p}$ orbital of barium is aligned along the relative velocity vector, as expected from the nature of the electron-transfer mechanism.

A simplified correlation diagram for the electronic configurations of interest for the $\mathrm{M}+\mathrm{Cl}_{2}$ reactions $(\mathrm{M}=\mathrm{Ca}, \mathrm{Ba}, \ldots)$ is shown in figure 26 , where the doubly ionic potential correlating the $\mathrm{M}^{+}+\mathrm{Cl}_{2}^{-}$ionic state to the $\mathrm{MCl}^{+}+\mathrm{Cl}^{-}$chemi-ionization channel has been omitted for clarity. The diagram allows one to follow the change of electron configurations along the reaction coordinate and to draw qualitative conclusions about the role of electronic excitation and orbital alignment. For instance, one can see how the $(1) \rightarrow\left(1^{\prime}\right)$ ground-state reaction is initiated by the electron transfer

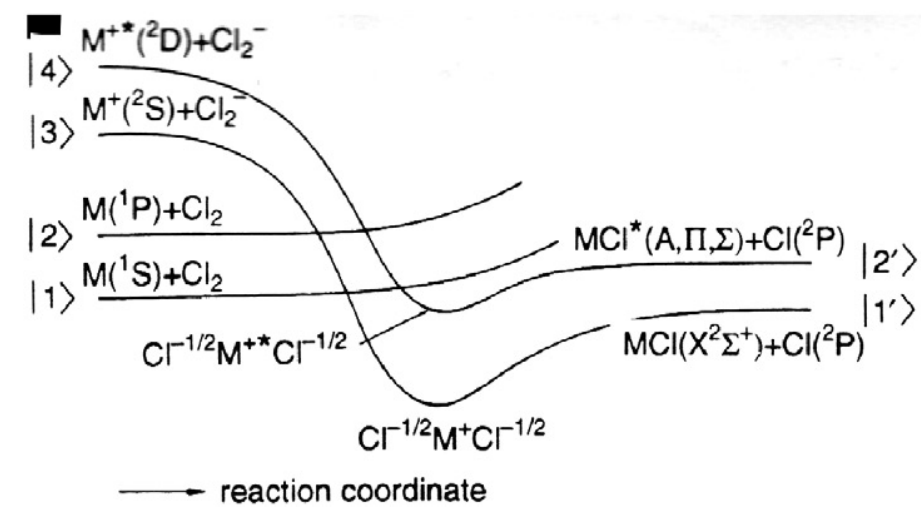

Figure 26. Correlation diagram for electronic configurations for $\mathrm{M}+\mathrm{Cl}_{2}$ reactions. Adapted from [110]. 
at the (1)*(3) crossing, e.g. the so-called 'outer' harpooning. Alternatively, the $(2) \rightarrow\left(2^{\prime}\right)$ chemiluminescence channel can be produced at the (2)*(4) 'inner' harpooning, by those trajectories which reject the $(2) *(3)$ intersection.

Apart from the necessary energy requirements, the final outcome depends on the particular geometry of the reagents as they correlate with the desired product channels. For the $\mathrm{Ba}^{+}+\mathrm{Cl}_{2}^{-}$charge-transfer channel, the interaction proceeds through the $(2)^{*}(3)$ intersection. In this case, when the p orbital is parallel to the relative velocity vector, the covalent surface and the ionic surface are of the same ${ }^{2} \Sigma$ symmetry, so that their interaction leads either to the $\mathrm{BaCl}\left(\mathrm{X}^{2} \Sigma^{+}\right)+\mathrm{Cl}\left(3 \mathrm{p}^{5}{ }^{2} \mathrm{P}\right)$ ground-state reaction products or to the $\mathrm{Ba}^{+}\left(6 \mathrm{~s}{ }^{2} \mathrm{~S}\right)+\mathrm{Cl}_{2}^{-}$charge-transfer products. On the other hand, when the p orbital is perpendicular to the relative velocity vector, the two surfaces are of different symmetry, so that they cross without interaction, hence a depletion of the $\mathrm{Ba}^{+}$channel and an enhancement of the (2)*(4) inner harpooning occurs. The intersection at $(2) *(3)$ thus allows the system to pass without electron transfer and to penetrate towards the (2)*(4) inner harpooning leading to chemiluminescence emission.

One of the clear consequences of the above discussion is the complementarity of the two channels, the one initiated by (1)*(3) outer harpooning leading to groundstate reaction products, chemi-ionization and charge-transfer products and the other initiated by $(2) *(4)$ inner harpooning leading to chemiluminescent products. These results are complementary to those of Zare and coworkers [3] on the $\mathrm{Ca}\left(4 \mathrm{p}^{1} \mathrm{P}_{1}\right)+\mathrm{HCl} \rightarrow \mathrm{CaCl}\left(\mathrm{A}^{2} \Pi, \mathrm{B}^{2} \Sigma^{+}\right)+\mathrm{H}$ reactions, where a dependence of the chemiluminescent yield on alignment of the atomic orbital was observed for the first time, as is shown in figure 27.

It was found that a parallel alignment of the $\mathrm{p}$ orbital along the relative velocity vector enhances the formation of $\mathrm{CaCl}$ in the $\mathrm{B}^{2} \Sigma^{+}$state, whereas a perpendicular alignment favours formation of the $\mathrm{A}^{2} \Pi$ state. This can be understood within the framework of an electron jump model of collision by considering 'outer' and 'inner'

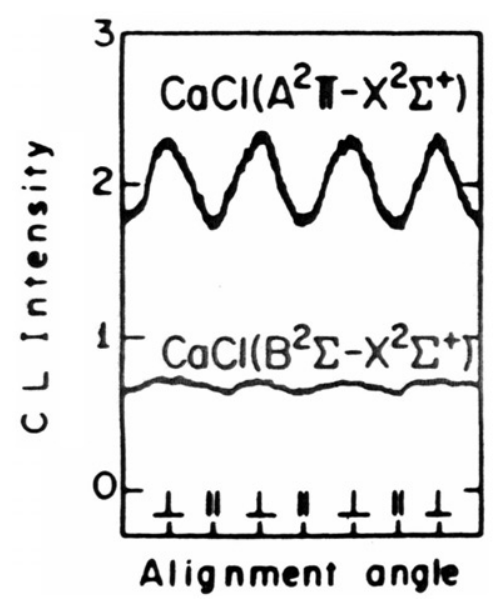

Figure 27. Chemiluminescence emission intensity in the $\mathrm{Ca}\left({ }^{1} \mathrm{P}_{1}\right)+\mathrm{HCl}$ reaction, as a function of the laser-induced alignment of the Ca p orbital. Adapted from [3]. 


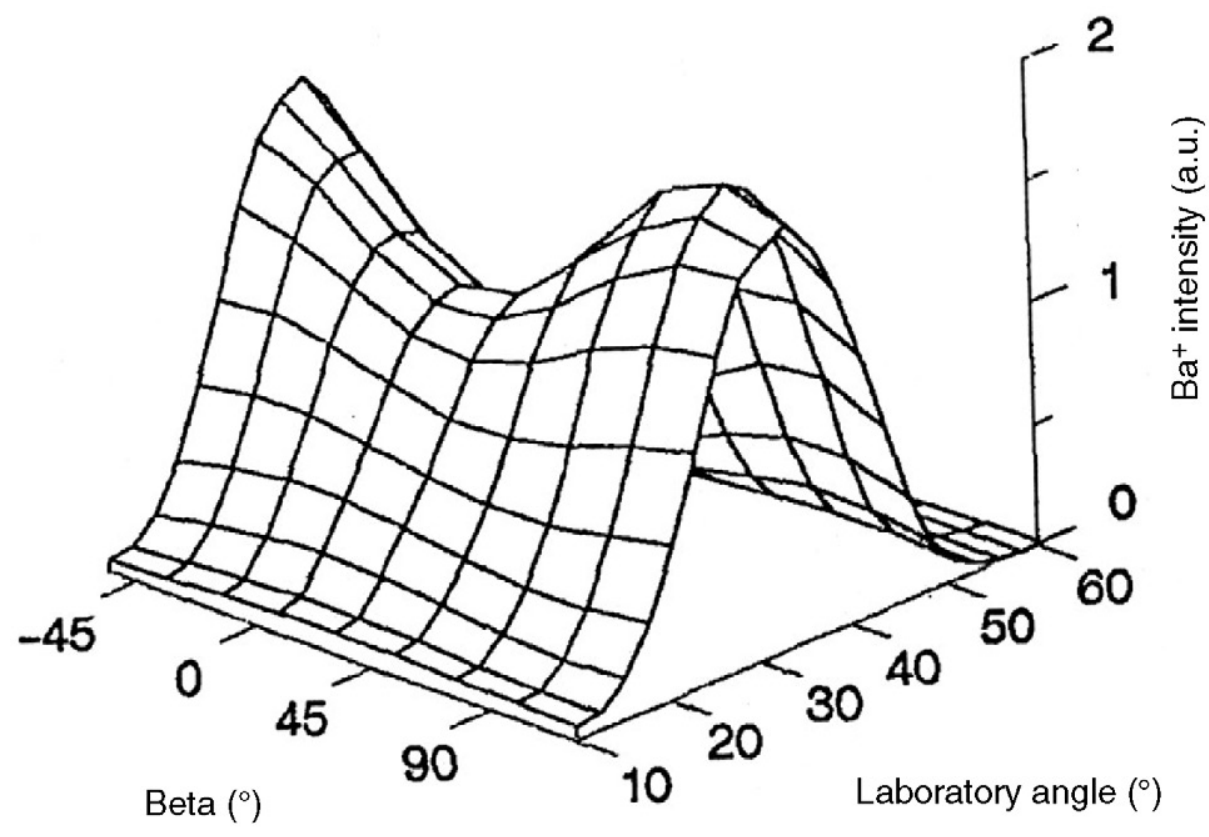

Figure 28. Alignment angular distribution of the $\mathrm{Ba}^{+}$intensity produced in the $\mathrm{Ba}+\mathrm{Br}_{2}$ reaction at a collision energy of $1.6 \mathrm{eV}$. This figure is reproduced with permission from [111].

harpooning, the first the most efficient being independent of orbital alignment, whereas the second is not. In this model, the symmetry of the reagent system is conserved, the $\mathrm{p}$ orbital of the $\mathrm{Ca}$ atom transforms into a $\mathrm{CaCl}$ molecular orbital and its alignment serves to orientate the reaction products towards the $\mathrm{B}^{2} \Sigma^{+}$the $\mathrm{A}^{2} \Sigma$ state.

The ion-pair formation in crossed-beam $\mathrm{Ba}\left({ }^{1} \mathrm{P}_{1}\right)+\mathrm{Br}_{2} \rightarrow \mathrm{Ba}^{+}+\mathrm{Br}_{2}^{-}$reaction has been studied by Suits et al. [111] with the finding of a strong orbital alignment for $\mathrm{Ba}^{+}$ production. As shown in figure 28, the peak of this dependence changes strongly with scattering angle for alignment of the $\mathrm{p}$ orbital in the scattering plane, showing the maximum flux for perpendicular alignment with respect to the relative velocity vector.

The reaction of ground state barium with $\mathrm{Br}_{2}$ at thermal energies gives predominantly $\mathrm{BaBr}+\mathrm{Br}$ through the harpoon mechanism. The excitation of $\mathrm{Ba}$ to the $\left({ }^{1} \mathrm{P}_{1}\right)$ state lowers its ionization potential from 5.2 to $3.0 \mathrm{eV}$ and, therefore, moves the first crossing for the $\mathrm{Ba} \cdot \cdots \mathrm{Br}_{2}$ system from 4 to $12 \AA$. In addition, the laser excitation of the $\mathrm{Ba}$ atom changes the symmetries of the diabatic surfaces resulting into three distinct potentialenergy surfaces for the $\mathrm{Ba}\left({ }^{1} \mathrm{P}_{1}\right)+\mathrm{Br}_{2}$ and their intersection with the $\mathrm{Ba}^{+}\left({ }^{2} \mathrm{~S}\right)-\mathrm{Br}_{2}{ }^{-}$.

The different reaction geometries are shown schematically in figure 29 [112] for $\mathrm{C}_{\infty v}$ and $\mathrm{C}_{2 v}$ geometries. The outer crossing is represented by a conical intersection and consequently the probability of the electron transfer is sensitive to alignment of the barium p orbital as experimentally depicted in figure 28 .

Looking at the three possible reaction geometries illustrated in figure 29 it can be seen that the two favourable configurations for the electron transfer, the non-adiabatic transition, are $\Sigma$ in $\mathrm{C}_{\infty v}$ and $\mathrm{B}_{1}$ in $\mathrm{C}_{2 v}$. 


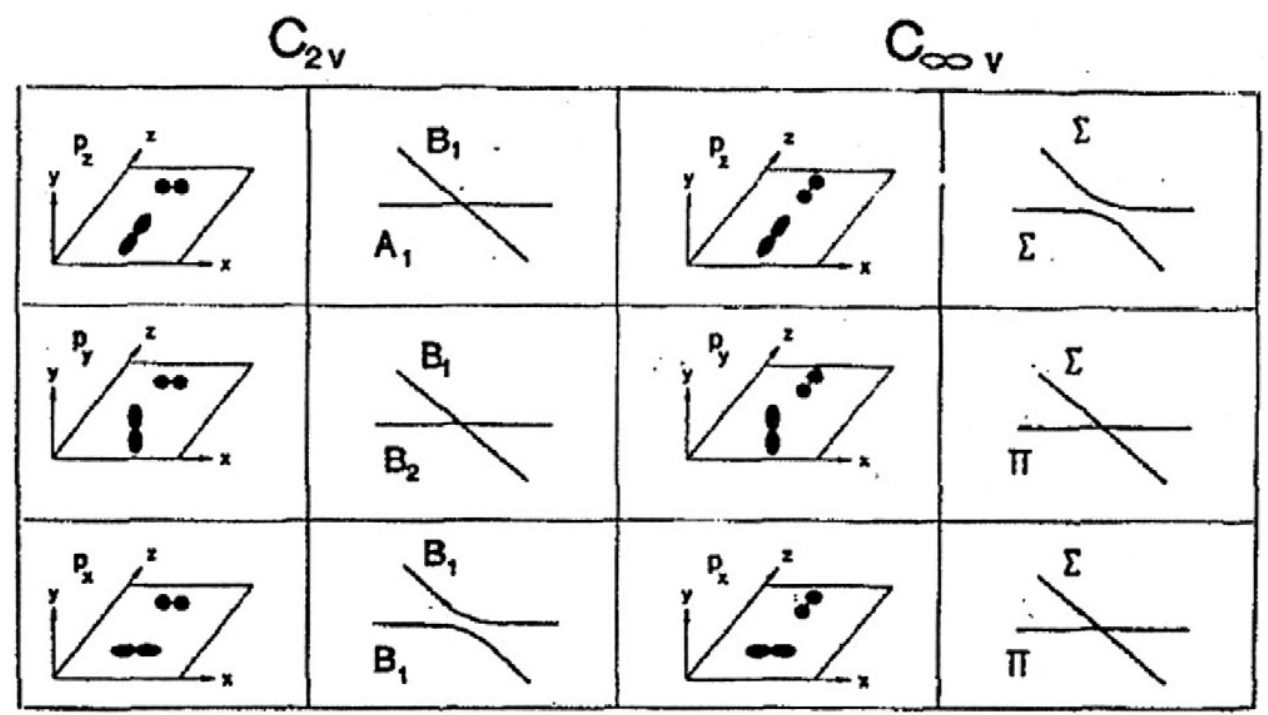

Figure 29. Schematic illustration of possible reaction geometries (adapted from [112]).

Looking at the data for in-plane rotation of the $\mathrm{p}$ orbital and considering that most likely the initial and final nuclear orbital angular momentum vectors to be parallel, then the two favourable configuration $\Sigma$ in $(\infty, v)$ and $\Pi$ in $C_{2 v}$ require the impact parameter to lie in the scattering plane. Furthermore all $\mathrm{Ba}^{+}$is forward or forward-sideways scattered, which is a clear indication of large impact parameter collision dominating the charge transfer process.

The alignment dependence of the charge transfer $\mathrm{Ba}^{*}+\mathrm{Br}_{2} \rightarrow \mathrm{Ba}^{+}+\mathrm{Br}_{2}^{-}$from out-of-plane data also suggests that this charge-transfer process is dominated by large impact parameter collisions that achieve collinear nuclear geometry and $\Sigma$ orbital alignment at the crossing seam.

The relationship between the centre-of-mass and body-fixed frames depends upon the impact parameter value. At small impact parameters parallel, $\sigma$ alignment, in the c.m. transforms to parallel body-fixed $\Sigma$ alignment. However, at larger impact parameters the $\sigma$ alignment may give rise to parallel $\Sigma$ or perpendicular $\Pi$ alignment depending upon the degree of adiabaticity. The non-adiabatic behaviour implies a non-adiabatic transition at which point $\Omega$ (the projection of the electronic orbital angular momentum on the body-fixed axis) changes, i.e. $|\Delta \Omega|=1$. Adiabatic behaviour implies $\Delta \Omega=0$, i.e. the conservation of $\Omega$ as the reagents approach. The latter situation is an example of the so-called 'orbital following' behaviour which has been tested in many atom-atom collisions [113].

The occurrence of 'orbital following', also referred to as orbital locking phenomena, depends on the magnitude of the splitting between the $\Sigma$ and $\Pi$ energy curves. In other words, it depends on whether $\Omega$ is a good quantum number or not. In general, orbital locking is taking place when $\Omega$ splitting is large relative to the angular velocity of the collision. 
A direct procedure to probe the vectorial nature of elementary chemical reactions is to measure the reactivity as a function of the reagents' orientation. One of the most used methods to orient a molecule in a beam has been the electrostatic hexapole technique [114]. Janssen et al. [115, 116] measured the steric effect as a function of the relative translational energy for some exit channel of the $\mathrm{Ca}\left({ }^{1} \mathrm{D}\right)+\mathrm{CH}_{3} \mathrm{X}$ $(j, k, m=1,1,1) \rightarrow \mathrm{Ca} \mathrm{X}\left(\mathrm{A}^{2} \Pi, \mathrm{B}^{2} \Sigma^{+} \mathrm{A}^{\prime}{ }^{2} \Delta\right)+\mathrm{CH}_{3}$ with $\mathrm{X}$ equal to $\mathrm{F}, \mathrm{Cl}$ or $\mathrm{Br}$. In these studies the steric effect for the $(j, k, m=1,1,1)$ rotational state is defined as the difference between the reactive cross-section for favourable conditions (i.e., those at which the $\mathrm{X}$ atom points toward the $\mathrm{Ca}$ atom) and the reactive cross-section for unfavourable conditions (i.e. $\mathrm{CH}_{3}$ group pointing toward the $\mathrm{Ca}$ atom), normalized to the total reactive cross-section for unoriented molecules. The experimental results showed negative steric effects. The steric effect was found to increase with collision energy.

A well-known theoretical model to analyse orientational effects in chemical reactions is that of angle dependent line of centres (ADLC). In this model the reagent molecule is surrounded by an energy barrier. Thus reaction occurs if enough radial kinetic energy is available to surmount the barrier. This barrier can be taken to be proportional to the cosine of $\theta_{\mathbf{R}}$ which is the angle between the symmetry axis of the molecule and the intermolecular axis. The ADLC model predicts a decreasing steric effect when the collision energy is increased, because as the collision energy increases a wider range of $\theta_{\mathrm{R}}$ is allowed for reaction. The experimentally found negative effect cannot be explained by the standard ADLC model.

The energy dependence of the steric effect for the reactions $\mathrm{Ca}\left({ }^{1} \mathrm{D}\right)+\mathrm{CH}_{3} \mathrm{X}$ $(j, k, m=1,1,1) \rightarrow \mathrm{Ca} \mathrm{X}+\mathrm{CH}_{3}$ with $\mathrm{X}=\mathrm{F}, \mathrm{Cl}, \mathrm{Br}$ has been investigated by Mejier et al. [117] using a semiclassical method. The relative motion of $\mathrm{Ca}$ with respect to $\mathrm{CH}_{3} \mathrm{X}$ was treated classically, while the rotation of the $\mathrm{CH}_{3} \mathrm{X}$ molecule and the electronic state of $\mathrm{Ca}$ and $\mathrm{CH}_{3} \mathrm{X}$ were treated quantum mechanically. One of the key ingredients of the model is the correlation of the projection of the electronic angular momentum on the intermolecular axis with the projection of the electronic orbital angular momentum on the diatomic axis - an idea first suggested by Menzinger [110, 118]. This correlation model together with the semiclassical method reproduced satisfactorily the steric effect for the $\mathrm{CaX}$ channels of the above reactions. To reproduce the experimental steric effects for these three reactions a 'cone of acceptance' with cutoff angles of $105^{\circ}, 150^{\circ}$ and $140^{\circ}$ was used for the $\mathrm{X}=\mathrm{F}, \mathrm{Cl}, \mathrm{Br}$ reactions, respectively. It was pointed out that the increase in the cutoff angle, going from $\mathrm{CH}_{3} \mathrm{~F}$ to $\mathrm{CH}_{3} \mathrm{Cl}$ and $\mathrm{CH}_{3} \mathrm{Br}$ is a consequence of the fact that $\mathrm{Cl}$ and $\mathrm{Br}$ are larger than $\mathrm{F}$. The difference in cutoff angle between $\mathrm{Ca}+\mathrm{CH}_{3} \mathrm{Cl}$ and $\mathrm{Ca}+\mathrm{CH}_{3} \mathrm{Br}$ reactions was attributed to some differences in the (short-range) dynamics not included in the employed reaction model.

3.3.2 Product energy disposal and electronic branching ratio. Ground state alkalineearth reactions with halogen compounds were studied in the early days of reaction dynamics by the molecular beam method [119, 120]. Reactive scattering of $\mathrm{Ca}, \mathrm{Sr}$ and $\mathrm{Ba}+\mathrm{CH}_{3} \mathrm{I}$ studied by Lin et al. [119] indicated predominant backward scattering with significant product translational energy disposal as corresponds to a direct impulsive mechanism governed by a repulsive surface. Detailed studies of $\mathrm{Ba}\left({ }^{1} \mathrm{~S}_{0}\right)+\mathrm{C}_{n} \mathrm{H}_{2 n+1} \mathrm{Br}$ 
( $n=1,2,3,4,5)$ conducted by Han et al. [121] found that the $\mathrm{BaBr}$ product vibrational excitation increases slightly with the number of carbon atoms of the alkyl group. On the other band, in the reactions of $\mathrm{Ba}+(\mathrm{n}, \mathrm{i}, \mathrm{s}, \mathrm{t}) \mathrm{C}_{4} \mathrm{H}_{9} \mathrm{Br}$ the $\mathrm{BaBr}$ excitation energy is almost identical regardless of the reactant isomer $\mathrm{C}_{4} \mathrm{H}_{9} \mathrm{Br}$ employed [121].

Since the discovery of $\mathrm{Ba}+\mathrm{Cl}_{2}$ chemiluminescence, electronic product state distributions have been measured in many reactions of metal atoms with halogen and oxygen-containing molecules. In the alkaline-earth atom reactions with halogencontaining compounds it was found that chemiluminescence yields were a few per cent and, more typically, a fraction of a per cent [122]. Furthermore, these electronic distributions decrease monotonically with available energy suggesting a statistical behaviour $[110,122,123]$ and that the electronic inversions necessary for laser action were elusive. This issue has been carefully analysed by Menzinger [110] using different reaction models and local state correlation diagrams.

As is well known, conical intersections at the outer harpooning regions manifest when the reagents have identical symmetry species (avoided crossing) in one or two point groups and different symmetry species (symmetry enforced crossing) in another point group. They are relevant for the chemiluminescent channels because they facilitate access to the inner harpooning region. This is the case for the ground state reaction $\mathrm{M}\left({ }^{1} \mathrm{~S}\right)+\mathrm{X}_{2}$, where a conical intersection occurs in $\mathrm{C}_{2 v}$ geometry. The outer harpooning region occurs close enough resulting in a strongly avoided intersection in $\mathrm{C}_{S}$ geometry. Consequently the harpooning takes place with a high probability at low collision energies and random geometry $\left(\mathrm{C}_{s}, \mathrm{C}_{\infty v}\right)$. As a result, the reactive flux is channelled toward the ground state and chemi-ion channels, except in perpendicular $\mathrm{C}_{2 v}$ collisions for which the conical intersection provides access to the inner harpooning region, i.e. the system is allowed to pass through this protective shell without an electron transfer and to proceed towards inner harpooning and chemiluminescence. Figure 30 illustrates this 'protective' outer harpooning shell, showing how only trajectories within a narrow cone surrounding $\mathrm{C}_{2 v}$ can penetrate. In conclusion, the low statistical weight of this $\mathrm{C}_{2 v}$ symmetric geometry is responsible for the low chemiluminescence yields.

\section{Half collisions: photo-induced intracluster reactions}

\subsection{Photo-induced intracluster reaction by nanosecond pump and probe techniques: the intracluster $\mathrm{Ba} \cdot \cdots \mathrm{FCH}_{3}+\mathrm{hv} \rightarrow$ product reaction}

Reactions of this type are, for example, initiated in van der Waals (vdW) clusters, $\mathrm{M} \cdots \mathrm{XR}\left(\mathrm{M}=\right.$ alkali metal, $\left.\mathrm{X}=\mathrm{F}, \mathrm{Cl}, \mathrm{Br}, \mathrm{R}=\mathrm{H}, \mathrm{CH}_{3}, \mathrm{Ph}\right)$, by absorption of visible light. This approach was pioneered [124] in the investigation of the $\mathrm{Hg} \cdot \cdots \mathrm{Cl}_{2}$ and the $\mathrm{Ca} \cdot \cdots \mathrm{HX}(\mathrm{X}=$ halogen $)$ complexes, monitoring the yield of electronically excited product, employing laser-induced fluorescence as the probing technique.

This vdW approach was extended to study alkali-metal atom 'harpooning' reactions with complexes of $\mathrm{Na}$ with $\mathrm{CH}_{3} \mathrm{Cl}, \mathrm{CH}_{3} \mathrm{~F}$ and $\mathrm{PhF}[125,126]$. In these investigations, the photo-depletion of the complexes through time-of-flight mass spectrometry (ToF-MS) was measured, obtaining interesting spectroscopic information about the electronically excited van der Waals complex potential. 

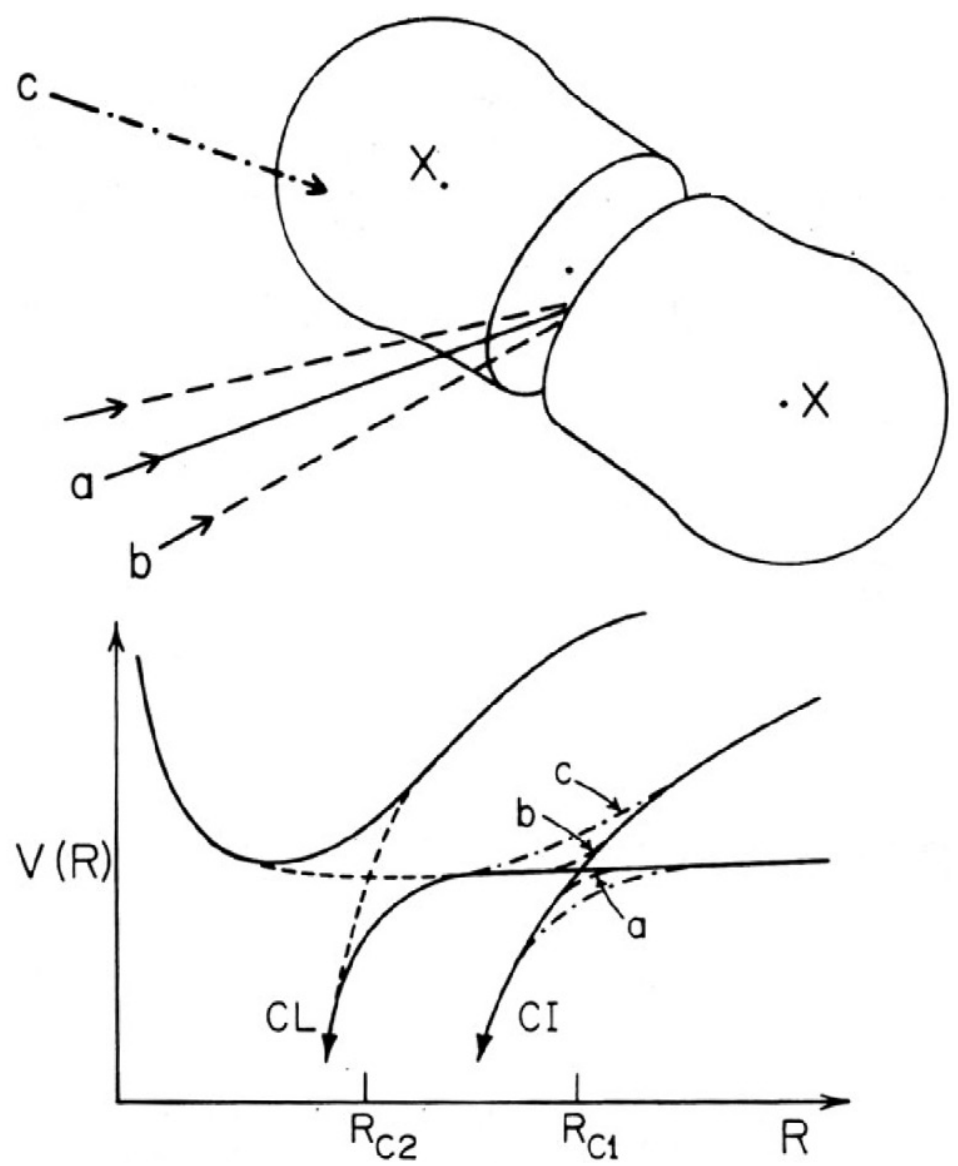

Figure 30. The conical intersection of the ${ }^{1} \mathrm{~A}_{1}$ and ${ }^{1} \mathrm{~B}_{2}$ surfaces at outer harpooning in $\mathrm{C}_{2 v}$ geometry adiabatic access to inner harpooning. For $\mathrm{C}_{s}$ collisions the avoided outer harpooning crossing acts as a protective shell against inner harpooning. The low chemiluminescence yield stems from the low statistical weight of $\mathrm{C}_{2 v}$ collision. Adapted from [110].

In this subsection we describe, in more detail, the $\mathrm{Ba} \cdot \cdots \mathrm{FCH}_{3}+h v \rightarrow$ product intracluster reaction, which has been extensively studied in frequency and time domain experiments.

Figure 31 shows a schematic energy diagram for the $\mathrm{Ba}+\mathrm{CH}_{3} \mathrm{~F} \rightarrow \mathrm{BaF}+\mathrm{CH}_{3}$ system. Inspection of this energy diagram reveals that the ground state reaction is exoergic. However, no $\mathrm{BaF}$ product from such a reaction has been reported. The absence of such a ground-state reaction in a full bimolecular collision is not surprising taking into account the energy requirement for the electron transfer, which presumably is the mechanism responsible for the reaction (the ionization potential for barium is rather high, namely $\mathrm{IP} \sim 5.21 \mathrm{eV}$, and the energy required to access the negative ionic potential of $\mathrm{CH}_{3} \mathrm{~F}$ is even higher).

A ToF mass spectrum of the $\mathrm{Ba}+\mathrm{FCH}_{3}$ system is shown in figure 32(a); for this, the fundamental output of the Nd:YAG laser is used for vaporization, and the 


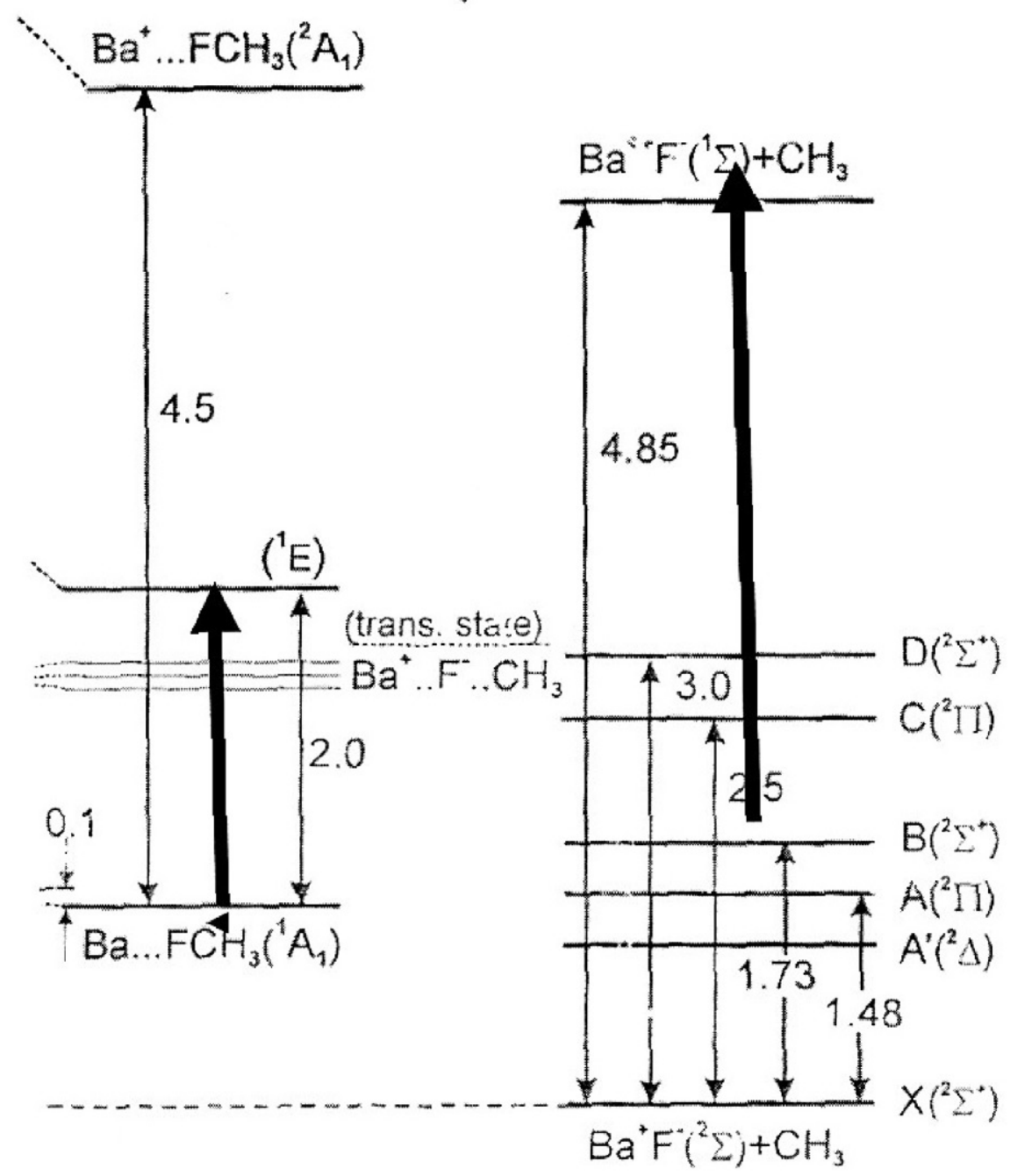

Figure 31. Energetic diagram for the $\mathrm{Ba}+\mathrm{CH}_{3} \mathrm{~F} \rightarrow \mathrm{BaF}+\mathrm{CH}_{3}$ system. The left column shows the barium electronic energy levels, and the BaF energy levels on the right; the centre column displays an approximate location of the $\mathrm{Ba} \cdot \cdots \mathrm{FCH}_{3}$ electronic states. This figure is reproduced with permission from [133].

species in the beam are ionized with the fourth harmonic $266 \mathrm{~nm}$ radiation. Strong depletion of the monomer signal is observed when the excitation laser, tuned to $547 \mathrm{~nm}$, is also allowed to enter the detection chamber (see the spectrum displayed in figure 32(b)). The disappearance of the $\mathrm{Ba} \cdot \cdot \cdot \mathrm{FCH}_{3}$ species was then monitored as a function of the dye laser wavelength. Figure 33 shows the photo-depletion spectrum for the $\mathrm{Ba} \cdot \cdots \mathrm{FCH}_{3}$ complex in the range $547-630 \mathrm{~nm}$, which exhibits vibrational structure with an energy spacing of about $150 \mathrm{~cm}^{-1}$, and large cross-sections $\left(\sim 60-70 \AA^{2}\right)$. 


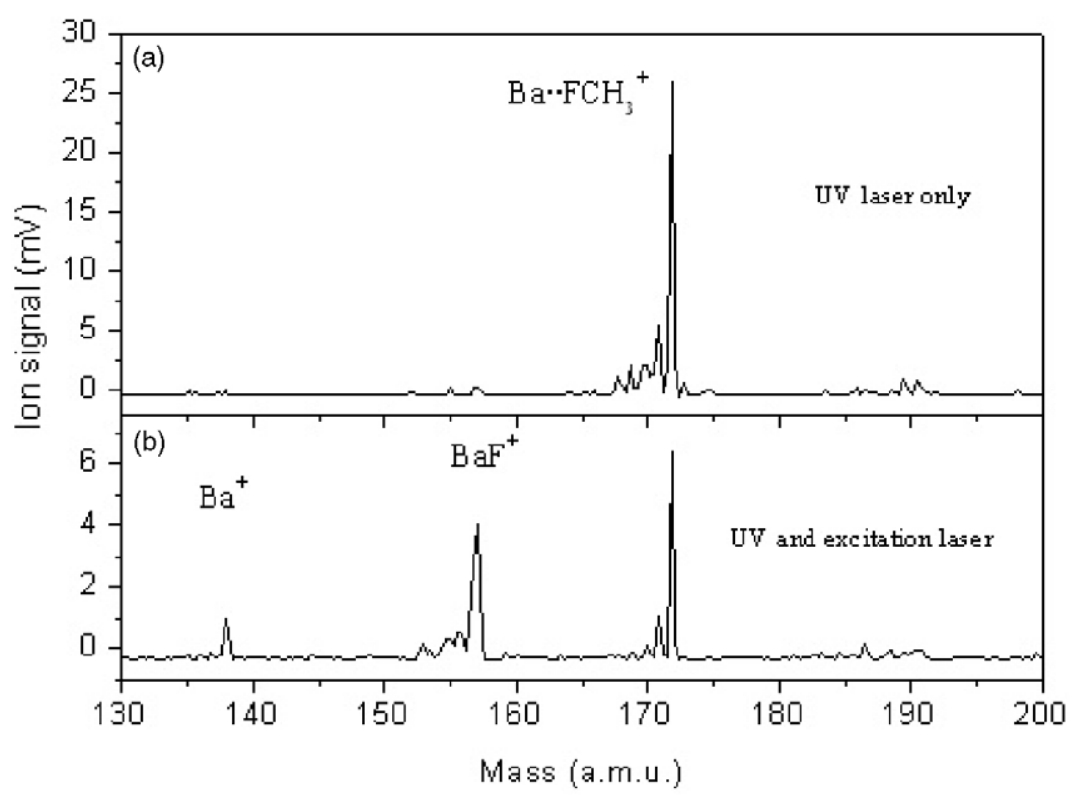

Figure 32. (a) Time-of-flight (ToF) mass spectrum of the $\mathrm{Ba}+\mathrm{CH}_{3} \mathrm{~F}$ system using the fundamental output of the Nd:YAG for Ba-vaporization and $266 \mathrm{~nm}$ laser radiation for ionization. (b) Same as in the top spectrum, but using additional laser excitation at $547 \mathrm{~nm}$ for $\mathrm{Ba} \cdot \cdots \mathrm{FCH}_{3}$ depletion. Tuning the dye laser to $547 \mathrm{~nm}$, depletion of the $\mathrm{Ba} \cdot \cdots \mathrm{FCH}_{3}$ complex is observed. Both $\mathrm{Ba}$ and $\mathrm{BaF}$ signals increase as a result of complex photo-fragmentation. Adapted from [129].

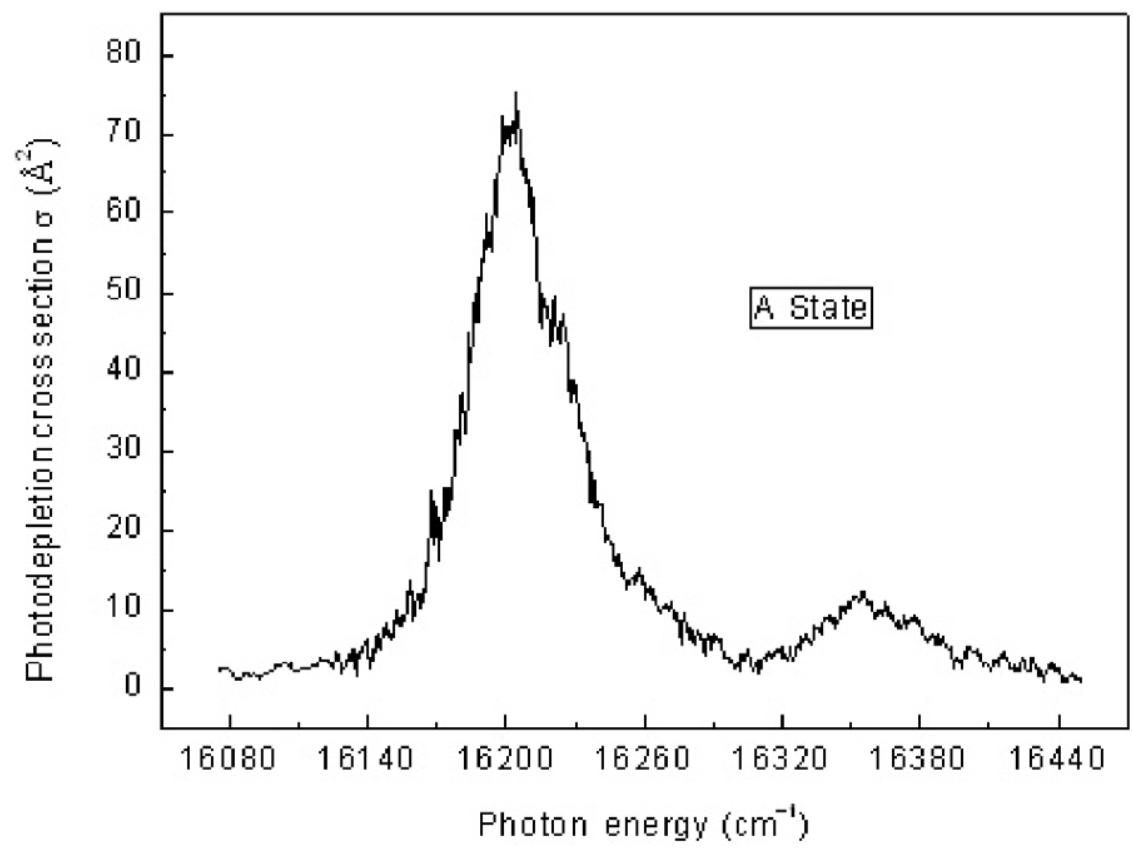

Figure 33. $\mathrm{Ba} \cdot \mathrm{F} \mathrm{FCH}_{3}$ photo-depletion spectrum in the range $547-630 \mathrm{~nm}$. Notice the double-peak structure with an energy spacing of $\sim 150 \mathrm{~cm}^{-1}$. This figure is reproduced with permission from [128]. 


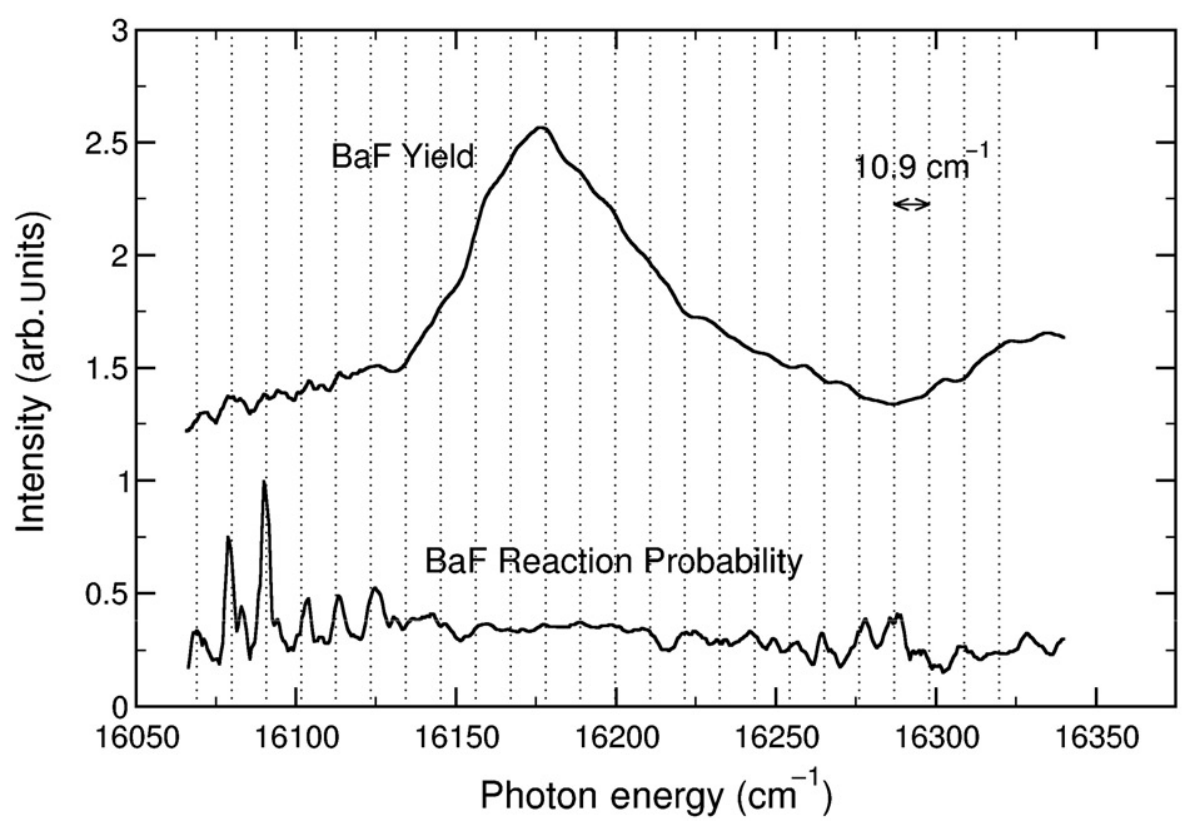

Figure 34. Top trace: $\mathrm{BaF}$ action spectrum from the laser intracluster reaction $\mathrm{Ba} \cdot \cdot \mathrm{FCH}_{3}+h v \rightarrow \mathrm{BaF}+\mathrm{CH}_{3}$. Notice the close resemblance with the photo-depletion spectrum shown in figure 33. Bottom trace: Energy dependence of the $\mathrm{BaF}$ reaction probability when the $\mathrm{Ba} \cdot \cdots \mathrm{FCH}_{3}$ complex is excited to its A-state. The ratio of the signal intensity of the top-panel spectrum to that of figure 33 is displayed as $\mathrm{BaF}$ reaction probability in the lower panel. Notice the energy spacing marked on the different peaks with the aid of vertical dashed-lines. Adapted from figure 17, p. 127 of [129].

A closer look at figure 32 reveals an increase in intensity of the $\mathrm{Ba}$ and $\mathrm{BaF}$ species, suggesting two open channels for the complex fragmentation, namely:

(a) Photo-induced charge-transfer reaction. In this scheme the absorbed photon induces the harpoon reaction, e.g.

$$
\mathrm{Ba} \cdots \mathrm{FCH}_{3}+h v \rightarrow\left|\mathrm{Ba} \cdots \mathrm{FCH}_{3}\right| \ddagger \rightarrow \mathrm{BaF}^{*}(\mathrm{BaF})+\mathrm{CH}_{3}
$$

leading to ground or electronically excited BaF.

(b) Breaking of the van der Waals bond. In this case the products are $\mathrm{Ba}$ and $\mathrm{CH}_{3} \mathrm{~F}$ with the $\mathrm{Ba}$ in its excited state, i.e.

$$
\mathrm{Ba} \cdots \mathrm{FCH}_{3}+h v \rightarrow \mathrm{Ba} \cdots \mathrm{FCH}_{3}^{*} \rightarrow \mathrm{Ba}^{*}+\mathrm{FCH}_{3} .
$$

The action spectra of these products can be obtained by monitoring the wavelength dependence of the $\mathrm{Ba}^{+}$and $\mathrm{BaF}^{+}$signals originating from the depletion of the parent complex. In the top panel of figure 34 the action spectrum for the BaF reaction channel is displayed; it was obtained by ionization with $266 \mathrm{~nm}$ photons. A resemblance of the $\mathrm{BaF}$ and the $\mathrm{Ba} \cdot \cdots \mathrm{FCH}_{3}$ photo-depletion signals is clearly manifested. 
The intensity in the action spectra, $I(v)$, can be described by

$$
I(v)=c \cdot|\langle\Psi(\mathrm{GS}) \mid \Psi(v)\rangle|^{2} \cdot A(v),
$$

where $c$ is a frequency-independent factor, and the squared term represents a FranckCondon factor between the complex groundstate (GS) and the excited state wave function at frequency $v$. The second factor, $A(v)$, can be considered as the coupling efficiency to the photo-fragmentation channel under consideration. Obviously, when several photo-dissociation channels are accessible, one has to consider a total coupling factor $A_{\text {tot }}(v)$, representing now the total photo-dissociation probability. Therefore, one needs to write the total photo-depletion spectrum, $I_{\mathrm{ph}}$, as

$$
I_{\mathrm{ph}}=c \cdot|\langle\Psi(\mathrm{GS}) \mid \Psi(v)\rangle|^{2} \cdot A_{\mathrm{tot}}(v)
$$

As a consequence, we can consider the (unnormalized) reaction probability, $P_{\mathrm{R}}^{\mathrm{i}}$, for the $i$ th photo-dissociation channel as given by

$$
P_{\mathrm{R}}^{\mathrm{i}}(v)=\frac{|\langle\Psi(\mathrm{GS}) \mid \Psi(v)\rangle|^{2} \cdot A^{\mathrm{i}}(v)}{\mid\left\langle\Psi(\mathrm{GS})|\Psi(v)|^{2} \cdot A_{\mathrm{tot}}(v)\right.}=\frac{A^{\mathrm{i}}(v)}{A_{\mathrm{tot}}(v)},
$$

in which $A^{\mathrm{i}}(v)$ stands for the coupling efficiency factor for the $i$ th photo-dissociation channel. Thus, $P_{\mathrm{R}}^{\mathrm{i}}$ can be obtained from the ratio of the two spectra, i.e. the action spectrum of channel $i$, and the total photo-dissociation spectrum. The ratio of these two spectra eliminates of the spectroscopic part (Franck-Condon dependence), leaving only the dynamic part contained in the reaction probability.

The energy dependence of the $\mathrm{BaF}$ reaction probability in the $A$-state can be estimated by using the last equation; it is displayed by the lower trace of figure 34 . Notice how the strong maximum in the centre of the $16175 \mathrm{~cm}^{-1} \mathrm{BaF}$ resonance (coincident with the maximum in the $\mathrm{Ba} \cdot \cdot \mathrm{FCH}_{3}$ photo-depletion spectrum) has disappeared in the $P_{\mathrm{R}}(E)$ plot. This indicates that the $\mathrm{BaF}$ maximum has its origin in the favourable Franck-Condon factors of photo-excitation. This is not the case for the peak structure observed in the low energy region of the BaF spectrum, which persists in the $P_{\mathrm{R}}(E)$ plot. Certainly, a clear peak structure is noticeable around the red part of the spectrum, i.e. near the threshold of the photo-depletion spectrum; these peaks are spaced by $\sim 10.9 \mathrm{~cm}^{-1}$. By measuring the reaction probability for the isotopic $\mathrm{Ba} \cdot \cdot \mathrm{FCD}_{3}+h v \rightarrow \mathrm{BaF}+\mathrm{CH}_{3}$, it can be shown [127] that this peak structure is due to torsional motion of the methyl group at the transition state region.

The decrease of the $\mathrm{Ba} \cdot \cdots \mathrm{FCD}_{3}^{+}$signal when excited with the dye laser was measured as a function of the excitation laser photon energy in the $16075-16380 \mathrm{~cm}^{-1}$ range, thus obtaining the photo-depletion spectrum of the weakly bound complex in its electronic A-state. The simultaneous measurement of the excitation laser energy, together with the determination of the $\mathrm{Ba} \cdot \cdot \mathrm{FCD}_{3}^{+}$signal with the UV laser only, 


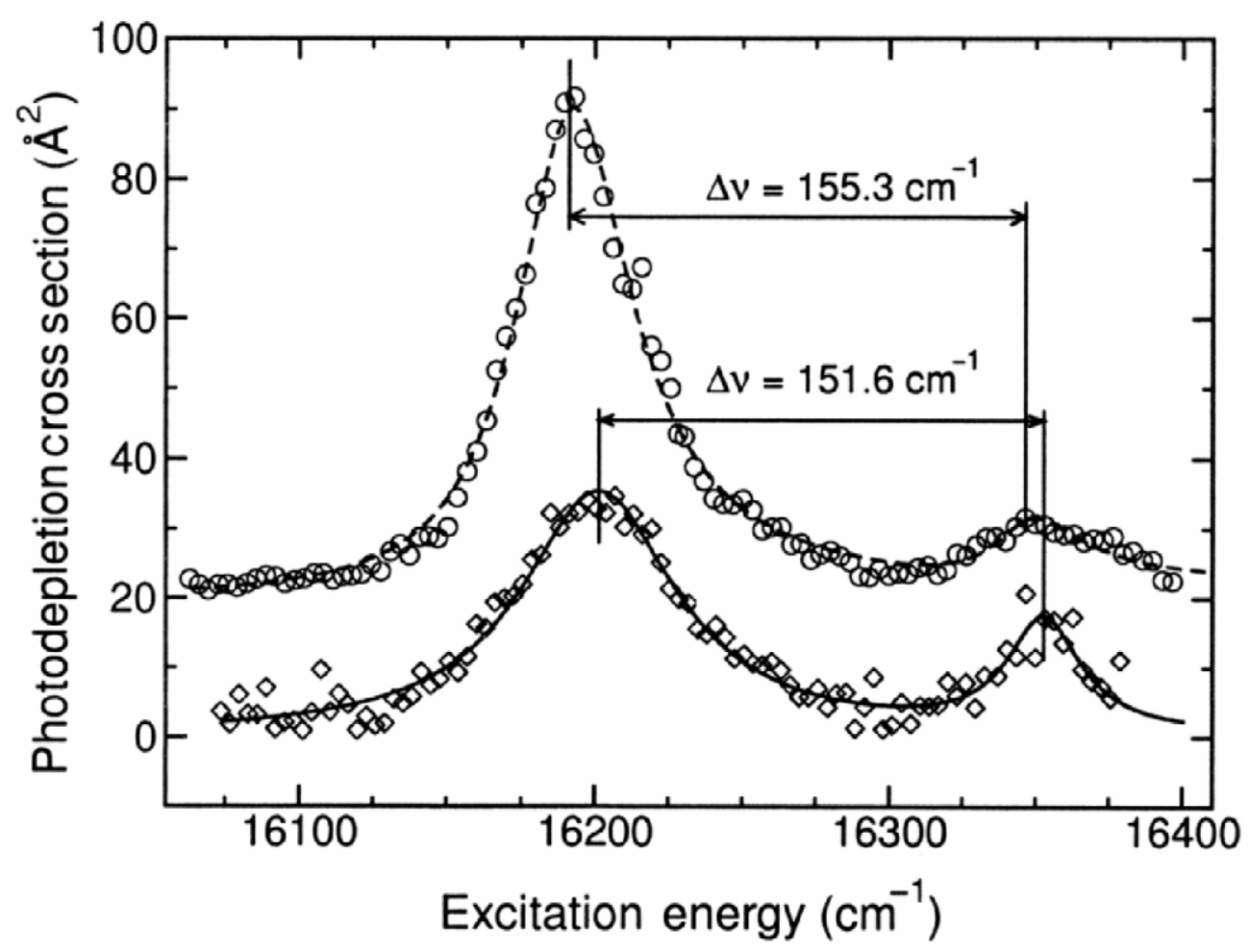

Figure 35. Photo-depletion cross-section for the $\tilde{\mathrm{A}}$-state of the $\mathrm{Ba} \cdot \cdots \mathrm{FCH}_{3}$ and $\mathrm{Ba} \cdot \cdots \mathrm{FCD}_{3}$ (diamonds, this work) complexes. The dashed line is a best fit to Fano profiles of the $\mathrm{Ba} \cdot \cdot \cdot \mathrm{FCH}_{3}$ data, and the straight line is a best fit to Lorentzian profiles of the $\mathrm{Ba} \cdot \cdots \mathrm{FCD}_{3}$ data. The $\mathrm{Ba} \cdot \cdot \mathrm{FCH}_{3}$ curves are offset by $+10 \AA^{2}$ for better clarity. The figure also shows the spacing between the two vibrational peaks for each complex, as obtained from the best fits. This figure is reproduced with permission from [127].

allowed us to determine the absolute photo-depletion cross-section, $\sigma$, as a function of the excitation energy. The resulting spectrum is shown in figure 35 (diamonds). The spectrum shows a structure very similar to the $\mathrm{Ba} \cdot \cdots \mathrm{FCH}_{3}$ (circles, data from [128]), but with a maximum photo-depletion cross-section of about $35 \AA^{2}$.

The energy dependence of the reaction probability may be obtained from the ratio of the product action spectrum of the channel under consideration, and the total photo-depletion spectrum of the parent molecule, as it was mentioned above.

The result is shown in figure 36 (lower part): the ratio of the $\mathrm{BaF}$ product signal to the $\mathrm{Ba} \cdot \cdots \mathrm{FCD}_{3}$ photo-depletion signal is shown. The upper part of figure 36 shows the relative reaction probability for the BaF channel for the photo-depletion of $\mathrm{Ba} \cdot \cdots \mathrm{FCH}_{3}$, for comparison. The same peak structure can be observed as for the deuterated case, but in this case with energy spacing of $10.9 \mathrm{~cm}^{-1}$ (see figure 34).

The energy spacing in the photo-depletion spectrum of $\mathrm{Ba} \cdot \cdot \mathrm{FCH}_{3}$ was assigned to the stretching mode of the $\mathrm{Ba} \cdot \cdots \mathrm{FCH}_{3}$ van der Waals mode being the experimental value of $155.3 \mathrm{~cm}^{-1}$ in good agreement with a theoretical calculated value of $145 \mathrm{~cm}^{-1}$ from Stert et al. [130, 131]. 


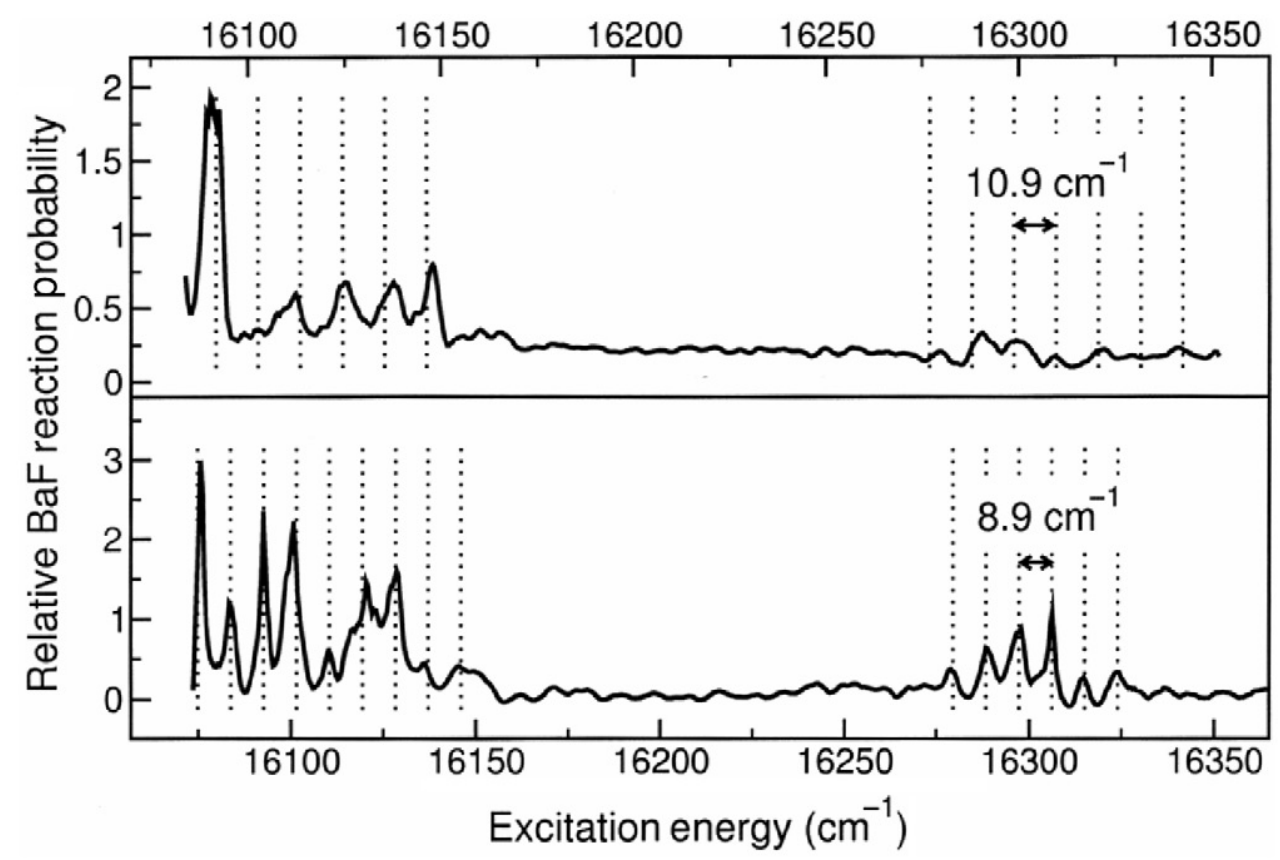

Figure 36. Upper part: Relative reaction probability for the reactive BaF product channel from $\mathrm{Ba} \cdot \cdots \mathrm{FCH}_{3}$ photo-depletion. The ratio of the product spectrum to the photo-depletion signal spectrum of the parent molecule is shown. Lower part: Relative reaction probability for the reactive BaF product channel from $\mathrm{Ba} \cdot \cdot \mathrm{FCD}_{3}$ photo-depletion. Both spectra show a clear peak structure which appears in the lower energy part of the two vibrational states. In the case of the $\mathrm{Ba} \cdot \cdot \mathrm{FCH}_{3}$, the peaks show an energy spacing of $10.9 \mathrm{~cm}^{-1}$ while in the case $\mathrm{Ba} \cdot \cdots \mathrm{FCH}_{3}$ the spacing is $8.9 \mathrm{~cm}^{-1}$. This figure is reproduced with permission from [127].

In this view, based on a simple harmonic oscillator model, the expected vibrational frequency for the isotopically substituted $\mathrm{Ba} \cdot \cdots \mathrm{FCD}_{3}$ should be

$$
v_{\mathrm{Ba} \cdots \mathrm{FCD}_{3}}=v_{\mathrm{Ba} \cdots \mathrm{FCH}_{3}} \sqrt{\frac{\mu_{\mathrm{Ba} \cdots \mathrm{FCH}_{3}}}{\mu_{\mathrm{Ba} \cdots \mathrm{FCD}_{3}}}} .
$$

The calculated value gives $\nu_{\mathrm{Ba}} \cdot . \mathrm{FCH}_{3}=150.17 \mathrm{~cm}^{-1}$ close to the experimentally found of $151.6 \mathrm{~cm}^{-1}$.

It is interesting to note the factor of two between the photo-depletion cross-section of both cluster isotopes. Though the $\mathrm{Ba} \cdot \cdots \mathrm{FCD}_{3}$ photo-fragmentation is slower than that of $\mathrm{Ba} \cdot \cdots \mathrm{FCD}_{3}$ both reactive and non-reactive fragmentation channels are in general much faster than the fluorescence channel. Thus the lower photo-fragmentation value of $\mathrm{Ba} \cdots \cdot \mathrm{FCD}_{3}$ reflects a lower excitation cross-section rather than a consequence of a slower photo-fragmentation processes.

The observed energy spacing of $10.9 \mathrm{~cm}^{-1}$ in the energy dependence of the reaction probability was initially associated to a $\mathrm{Ba} \cdot \cdot \mathrm{FCH}_{3}$ perpendicular transition. Since the $\mathrm{CH}_{3} \mathrm{~F}$ rotational constant with respect to rotation about its symmetry axis is 
$A=5.18 \mathrm{~cm}^{-1}$, a perpendicular transition $(\Delta K= \pm 1)$ would lead to a $\Delta v \cong 2 \mathrm{~A} \cong 10.36 \mathrm{~cm}^{-1}$ spacing. For the same reason one would expect an isotope effect of near 2 since the rotational constant $A$ for $\mathrm{FCD}_{3}$ is about $2.56 \mathrm{~cm}^{-1}$ which is clearly in contradiction with the present observation of $8.9 \mathrm{~cm}^{-1}$ energy spacing.

The simplest picture adopted to explain this contradiction involves the internal rotation of the $\mathrm{CH}_{3}$ or $\mathrm{CD}_{3}$ group which can be considered as approximately free rotation, and therefore the rotational energy $E$ (internal) is $F J(J+1)$. In this view the energy spacing between adjacent rotational levels is given by $\Delta E=2 F$.

In the framework of this simple rotation model the expected $\mathrm{F}$ value for the $\mathrm{Ba} \cdot \cdots \mathrm{F}$ $\mathrm{CD}_{3}$ could be calculation in a first approximation by assuming that the change due to the isotopic substitution mainly affects the moment of inertia of the $\mathrm{CX}_{3}(\mathrm{X}=\mathrm{H}, \mathrm{D})$ group. Furthermore, if one assumes that $\mathrm{CX}_{3}$ group acts a single particle and is situated at the same distance from the internal rotation axis, for both isotopes, the $\mathrm{F}$ branching ratio could be written as

$$
\frac{F_{\mathrm{CH}_{3}}}{F_{\mathrm{CD}_{3}}}=\frac{m_{\mathrm{CD}_{3}}}{m_{\mathrm{CH}_{3}}}=1.2
$$

The above results lead to $F_{\mathrm{CD}_{3}}=5.45 \mathrm{~cm}^{-1} / 1.2=4.54 \mathrm{~cm}^{-1}$.

Thus the calculated energy spacing in the $\mathrm{Ba} \cdot \cdot \mathrm{FCD}_{3}$ would be $2 \times 4.54=9.08 \mathrm{~cm}^{-1}$, which compares well with the experimentally found of $8.9 \mathrm{~cm}^{-1}$ in spite of the simplicity of the model and approximations made in the calculation.

The photo-induced intracluster $\mathrm{Ca}^{*}+\mathrm{CH}_{3} \mathrm{~F} \rightarrow \mathrm{CaF}^{*}+\mathrm{CH}_{3}$ reaction was studied [132] by exciting the $1: 1 \mathrm{Ca} \cdot \cdots \mathrm{CH}_{3} \mathrm{~F}$ complexes formed in a supersonic expansion. The transition state of the reaction was explored by monitoring the electronically excited product, $\mathrm{CaF}$, while scanning the laser that turns on the reaction. In addition, the electronic structure of the $\mathrm{Ca} \cdot \cdots \mathrm{FCH}_{3}$ system was studied using ab initio methods. These calculations provided structural information on the ground state of the $\mathrm{Ca} \cdot \cdots \mathrm{CH}_{3} \mathrm{~F}$ complex which is slightly bent, with calcium close to the $\mathrm{F}$ end of $\mathrm{CH}_{3} \mathrm{~F}$.

Two broad structured bands (named A and B hereafter) were found. They are extending quite far on each side of the calcium resonance line. The comparison between the experimental action spectrum and the calculated molecular curves helped to assign band $\mathrm{A}$ of the action spectrum to the excitation of molecular states that correlate to $4 \mathrm{~s} 3 \mathrm{~d}{ }^{1} \mathrm{D}$ at large separation between $\mathrm{Ca}$ and $\mathrm{FCH}_{3}$, whereas band $\mathrm{B}$ is associated with the molecular states correlating to $4 \mathrm{~s} 4 \mathrm{p}{ }^{1} \mathrm{P}$. The $a b$ initio calculations allowed an interpretation of the experimental data in terms of two reaction mechanism, for band $\mathrm{B}$. The first one involves a partial electron transfer from $\mathrm{Ca}$ to $\mathrm{CH}_{3} \mathrm{~F}$. However, because the excited complex is not expected to dissociate directly as $\mathrm{CaF}+\mathrm{CH}_{3}$, the mechanism cannot be reduced to a simple harpooning process. Instead, an internal conversion is likely to occur prior to reaction. In a similar way as was found for the the $\mathrm{Ba} \cdot \cdots \mathrm{CH}_{3} \mathrm{~F}$ photo-fragmentation dynamics discussed above. No such electron transfer is implied in the second reaction mechanism that involves the deposition of two or more vibrational quanta in the $\mathrm{C}-\mathrm{F}$ stretch in addition to the electronic excitation. 


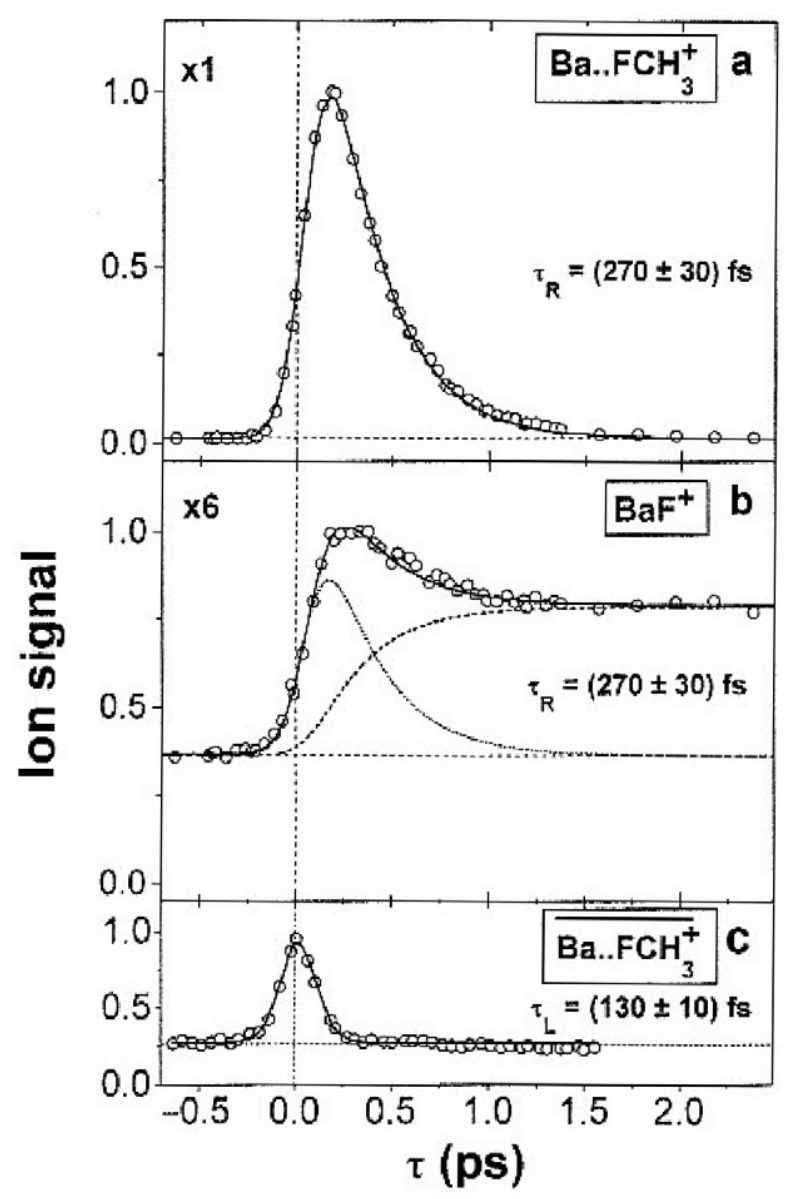

Figure 37. Ion signals for $\mathrm{Ba} \cdot \cdots \mathrm{FCH}_{3}$ (a) and $\mathrm{BaF}(\mathrm{b})$ as a function of the delay time $\tau$ between the pump pulse at $618 \mathrm{~nm}$ and probe pulse at $400 \mathrm{~nm}$. The solid lines represent the theoretical fit curves, which in figure (b) was obtained by superposition of two contributions (dashed and dotted lines). In figure (c), the pump wavelength is tuned off-resonant to $636 \mathrm{~nm}$, thus, the $\left(\mathrm{Ba} \cdot \cdot \mathrm{FCH}_{3}\right)^{+}$signal represents the cross correlation curve of the laser pulses for the width $\tau_{\mathrm{L}}=130 \mathrm{fs}$. This figure is reproduced with permission from [133].

\subsection{Photo-induced intracluster reactions by femtosecond pump and probe studies: the $\mathrm{Ba} \cdot \cdots \mathrm{FCH}_{3}+\mathrm{hv} \rightarrow$ product reactions}

Figure 37 shows the time behaviour of the $\mathrm{BaF}$ reaction product obtained in a pump and probe femtosecond experiment [133]. The $\mathrm{Ba} \cdot \cdots \mathrm{FCH}_{3}$ complex was excited at $618 \mathrm{~nm}$, and probed at a wavelength of about $400 \mathrm{~nm}$. The best fit (solid line) is the sum of two contributions. Firstly, after excitation of the parent complex, the BaF product is formed once the electron transfer has taken place. Thus, the $\mathrm{BaF}$ formation is expected to occur with a time delay, $\tau_{\mathrm{D}}$, identical to the time constant of complex fragmentation, i.e. $\sim 270 \mathrm{fs}$. The dashed line in figure 37 represents this contribution. An additional, a faster contribution may result from partial fragmentation of the parent ion 


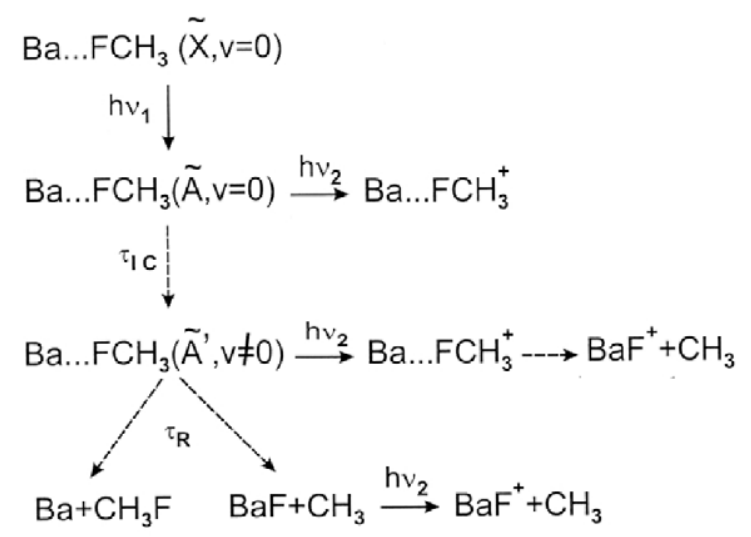

Figure 38. Reaction mechanism suggested for the intracluster reaction $\mathrm{Ba} \cdot \cdots \mathrm{FCD}_{3}^{*}$. This figure is reproduced with permission from [130].

(dotted line). Consequently, this contribution vanishes for delay times for which the parent ion signal has diminished to zero. The lower panel of figure 37 shows the $\mathrm{Ba} \cdot \cdots \mathrm{FCD}_{3}^{+}$signal when the pump laser is tuned off-resonance, to $630 \mathrm{~nm}$; thus the observed complex signal represents the cross-correlation curve of the laser pulses, which for the experiment discussed here was of the order $130 \mathrm{fs}$.

The mechanism suggested [131] for the scheme of the intracluster reaction in the $\mathrm{Ba} \cdot \cdots \mathrm{FCH}_{3}$ complex, initiated by excitation to its electronic $\tilde{A}$-state, is shown in figure 38 . Once this excited state is formed, reaction proceeds by internal conversion to the next lower electronic $\tilde{A}^{\prime}$-state. A direct proof of the energy transfer due to $\tilde{A} \longleftrightarrow \tilde{A}^{\prime}$ internal conversion can be obtained by time-resolved photo-electron spectroscopy, as outlined below. After the vibrationally excited $\tilde{A}^{\prime} m$-state is formed, the $v_{4}$-stretch mode of the $\mathrm{CH}_{3} \mathrm{~F}$ part acts as promoting mode, since the $\mathrm{C}-\mathrm{H}$ stretching energy is in near-resonance with the energy gap between the $\tilde{A}$ and $\tilde{A}^{\prime}$ states. This excitation energy is transferred to the $\mathrm{C}-\mathrm{F}$ bond to overcome the energy barrier for the electron jump from the $\mathrm{Ba}$ to the $\mathrm{FCH}_{3}$ part. The $\mathrm{BaF}$ formation (step 3 in the reaction scheme) competes with step 2 , i.e., the $\mathrm{Ba} \cdot \cdots \mathrm{FCD}_{3}^{+}$photo-dissociation after internal $\tilde{A} \rightarrow \tilde{A}^{\prime}$ conversion.

The need for vibrational energy to overcome the reaction barrier is clear because the laser excitation promotes the complex into the $v^{\prime}=0$ level of the electronically excited state. Therefore, as illustrated in figure 39, no energy is available to surmount the reaction threshold present along the $\mathrm{Ba}-\mathrm{C}$ coordinate. On the other hand, since the reaction takes place and the $\mathrm{BaF}$ is clearly detected, the reaction mechanism described in figure 38 was proposed. After the excitation process, step 1 of figure 38, a non-adiabatic $\tilde{A} \rightarrow \tilde{A}^{\prime}$ transition occurs, in which the $\tilde{A}^{\prime}$ state is formed with significant energy excess (exactly $0.35 \mathrm{eV}$ ). This electronic-to-vibrational energy transfer is mostly channelled into the vibrational $\mathrm{C}-\mathrm{H}$ stretching due to the near-resonance character of this energy transfer, i.e., $0.35 \mathrm{eV}\left(\equiv 2692 \mathrm{~cm}^{-1}\right)$ with the vibrational energy of the $\mathrm{C}-\mathrm{H}$ stretch.

How can this internal conversion process be proved? One way is to use femtosecond time-resolved electron spectroscopy. Here the probe laser produces both ions 


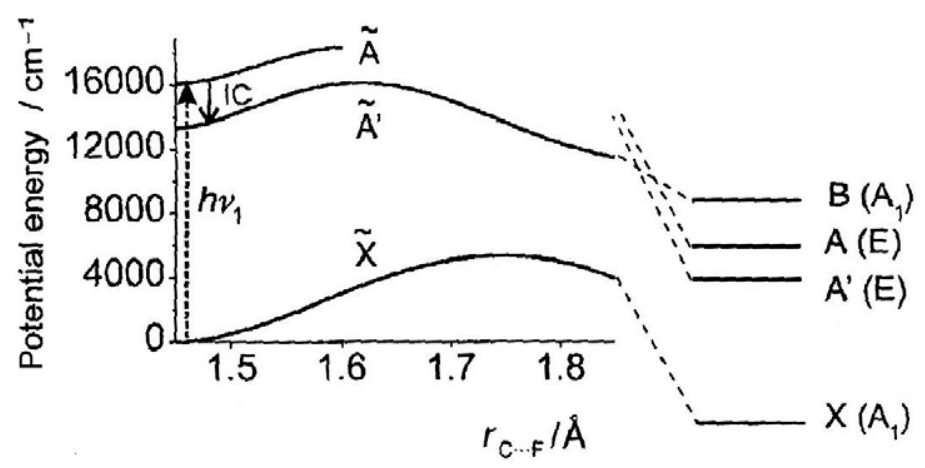

Figure 39. Ab initio potential of the $\mathrm{C} \cdot \cdots \mathrm{F}$ coordinate in the ground and excited state. Notice the energy barrier in both $\bar{X}$ and $\tilde{A}^{\prime}$ potential curves as the $\mathrm{C}-\mathrm{F}$ distance increases. This figure is reproduced with permission from [130].

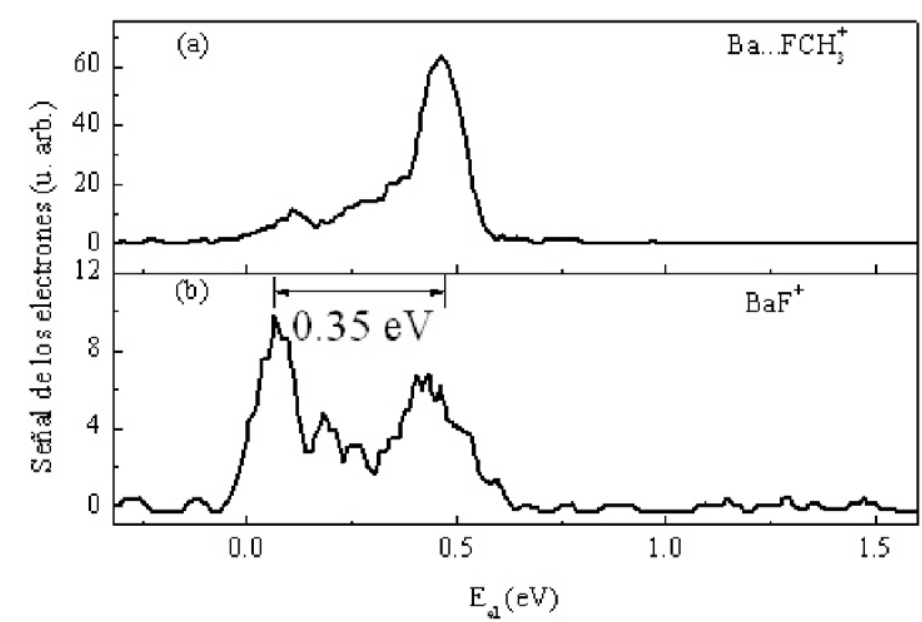

Figure 40. $\mathrm{Ba} \cdot \cdots \mathrm{FCD}_{3}^{+}$and $\mathrm{BaF}^{+}$photo-electron spectra, measured at a delay time of $200 \mathrm{fs}$. This figure is reproduced with permission from [131].

and electrons, and both signals are detected in coincidence using opposite-positioned time-of-flight spectrometers.

We shall keep in mind that in this process energy balance ensures the relationship

$$
E_{\mathrm{i}}\left(\mathrm{AB}^{+}\right)=h v_{\text {ionization }}-\mathrm{IP}-\mathrm{KE}_{\mathrm{e}}
$$

Thus, if one measures the kinetic energy, $\mathrm{KE}_{\mathrm{e}}$, of the electron after the photo-ionization of the AB-molecule with a photon of energy $h v_{\text {ionization, one can determine the internal }}$ energy of the ion. However, its ionization potential, IP has to be known. Figure 40 shows the $\mathrm{Ba} \cdot \cdots \mathrm{FCD}_{3}^{+}$and $\mathrm{BaF}^{+}$photo-electron spectra, which were measured at a delay time of $200 \mathrm{fs}$ [131]. 
As already shown in the reaction mechanism, at this delay time, the $\mathrm{Ba} \cdot \cdots \mathrm{FCD}_{3}^{+}$ signal is near maximum, and the $\mathrm{BaF}^{+}$signal is dominated by the contribution from $\mathrm{Ba} \cdots \mathrm{FCD}_{3}^{+}$fragmentation. The peak at $E_{\mathrm{el}}=0.45 \mathrm{eV}$ in the electron spectrum of $\mathrm{Ba} \cdot \cdots \mathrm{FCD}_{3}^{+}$is only $0.15 \mathrm{eV}$ below the maximum possible electron energy $E_{\text {el,max }}=h v_{1}+h v_{2}-$ I.P (with I.P $=4.5 \mathrm{eV}$ ), i.e. the vibrational energy in the ion state is restricted to values below $0.15 \mathrm{eV}$. The relatively narrow width $(\approx 0.1 \mathrm{eV})$ of this electron peak is consistent with a narrow Franck-Condon region for nearly vibrationless transitions. Thus, the primary excitation process leads to the vibrationless $\tilde{A}$ state of $\mathrm{Ba} \cdot \cdots \mathrm{FCD}_{3}^{+}$, followed by probe photon ionization to the $X\left({ }^{2} \mathrm{~A}_{1}\right)$ ground state of the ion, with only weak vibrational excitation. The dominant peak in the electron spectrum of $\mathrm{Ba} \cdot \cdots \mathrm{FCD}_{3}^{+}$corresponds to the energy of the primarily populated $\tilde{A}$ state. The partial transfer to the $\tilde{A}^{\prime}$ state, already complete at $\sim 200 \mathrm{fs}$ after the pump pulse, is scarcely observable for the parent ion (see the weak peak at $E_{\mathrm{el}}=0.1 \mathrm{eV}$ in figure 40). Because of the internal conversion the vibrational energy in the $\tilde{A}^{\prime}$ state is sufficiently high for almost complete dissociation into $\mathrm{BaF}$ and $\mathrm{CH}_{3}$. Hence, the electron signal corresponding to the $\tilde{A}^{\prime}$ state population of the parent complex will be observed in coincidence with the $\mathrm{BaF}^{+}$ion. Notice how the dominant peak in the electron spectrum of $\mathrm{BaF}^{+}$appears at $E_{\mathrm{el}} \approx 0.1 \mathrm{eV}$. The energy difference with respect to the main peak of the electron spectrum for $\mathrm{Ba} \cdot \cdots \mathrm{FCD}_{3}^{+}$is $0.33 \mathrm{eV}$; this energy is very close to the calculated energy gap between the $A$ and $A^{\prime}$ states, namely $0.35 \mathrm{eV}$. Thus, the key point of the reaction scheme is that the internal conversion from the initially excited $\tilde{A}$ state to the next lower $\tilde{A}^{\prime}$ state of the complex is directly confirmed as the rate-determining process for the laser-initiated reaction.

\section{Concluding remarks}

This review article has been centred in the reaction dynamics of alkaline-earth atoms plus molecules. Though these reactions have many similarities to those of corresponding alkali atoms, because of the low ionization potentials of the atomic reagents, they display distinct dynamical behaviour.

A clear example of such different reaction dynamics is due to the presence of two valence electrons in alkaline-earth atoms. We have seen several examples in which the possibility of a second electron transfer, the so-called inner harpooning, led to chemiluminescent channels with severe stereodynamical requirements mainly due to the statistically more favourable first electron transfer mechanism, the so-called outer harpooning mechanism, which normally leads to ground state products. This review analysed in detail these two types of reaction mechanisms by discussing the reaction dynamic of electronically excited alkaline-earth atoms prepared with linear polarized laser radiation and pointed out their main dynamical and stereodynamical features associated to each electron transfer process.

Clearly, the possibility of using laser spectroscopic techniques in the visible to analyse the internal product state distribution of the nascent alkaline-earth halide molecules has played a major role in unravelling the dynamics of these alkaline-earth atom reactions. Thus it was, for example, demonstrated how the electron transfer mechanism involves an early energy release leading to highly vibrationally excited products or to what extent 
the product rotational energy distribution is limited by the total angular conservation or by the energy conservation.

With some exceptions, propensity rules or general trends have been found associated to these excited harpooning reactions:

(i) From the point of view of the dynamics of electronically excited reagents, the adiabatic behaviour, exemplified by the conservation of the electronic angular momentum denoted by $\Omega$, i.e. $\Delta \Omega=0$, seems to be more often the case than otherwise.

(ii) The ultrashort time required for the electron transfer in many electronically excited alkaline-earth atoms reaction seems to be a dominant factor to prevail the occurrence of a non-statistical product energy distribution. Nevertheless, the requirement of a small but finite time for molecular rearrangement at the transition state configuration is otherwise necessary to account for the translational energy dependence of the reaction cross-section.

(iii) As far as product rotational energy partitioning is concerned, the dominant factor is obviously the transition state configuration depending on whether an insertion of abstraction mechanism is dominant. However, for heavy atom reactions the conservation of the total angular momentum imposes severe restrictions such as, for example, the well-known case of heavy + heavy-light reaction, a good example being that of the $\mathrm{Ba}+\mathrm{HI}$ reaction. Though in these cases the reagents' orbital angular momentum is mostly channelled into product rotational angular momentum, two distinct behaviours were found: whereas at low collision energies, the maximum reactive impact parameter, $b_{\max }$, is determined by an angular momentum (centrifugal) barrier, at collision energies, the conservation of energy determines the value of $b_{\max }$. These two processes were therefore identified as the mechanisms responsible for the bimodal character of the alkaline-earth halide product internal-state distribution.

(iv) The fact that alkaline-earth atoms have an $n s^{2}$ valence-shell electronic structure leads to the formation of species of the type $\mathrm{H}-\mathrm{M}-\mathrm{X}$, due to insertion of the metal atom into the hydrogen halide bond: they are stable and could be participating in a complex-mediated reaction mechanism. As a result two possibilities appear for the transformation from reactants to products in the reactions of alkaline-earth metal atoms with hydrogen halide molecules: a direct halogen abstraction pathway, which involves a harpoon mechanism, and an insertion pathway, in which the $\mathrm{H}-\mathrm{M}-\mathrm{X}$ potential well is sampled. In this review examples of each category have been discussed. It is interesting to find distinctly different pathways followed by the reactions of alkaline-earth atoms with hydrogen or methane. Despite existence of bimodal rotational distributions for the $\operatorname{Mg}\left(3^{1} \mathrm{P}\right)$ reactions, the microscopic pathways are dominated by the anisotropic interaction of the ground state surface. The bimodal feature depends on coupling in the exit channel. The $\mathrm{H}$-atom abstraction reactions encounter a substantial energy barrier in the entrance channel. These reactions $(\mathrm{Mg}$ and $\mathrm{Ca})$ fail to obey the harpooning mechanism either, except for high-lying $\operatorname{Mg}\left(4 \mathrm{~s}^{1} \mathrm{~S}_{0}\right.$, $3 \mathrm{~d}^{1} \mathrm{D}_{2}$ ) reactions. 
A substantial part of the review was dedicated to discussing the dynamics of the laser intracluster reactions, in particular that of the barium with methyl fluoride, extensively investigated by both nanosecond and femtosecond pump and probe techniques.

The preparation of the van der Waals molecule containing 1:1 metal atom and solvent molecule provides the ideal scenario to access the transition state region by laser excitation. Examples of the link between the transition state spectroscopy and the underlying intracluster reaction connecting the frequency domain with time-domain aspects of the photo-fragmentation dynamics for this alkaline-earth atom clusters were presented and discussed. Perhaps one of the most important conclusions in this subfield is the unprecedented resolution in investigating the detailed reaction mechanism for theses harpooning reactions when one uses photon-ion and photon-electron femtosecond pump and probe technique. Indeed the combination of theses fs techniques with nanosecond techniques and $a b$ initio calculations of the relevant potential energy curves of the excited cluster allowed us to demonstrate the complete reaction pathways of an ultrafast reaction, and more specifically the presence of non-adiabatic transitions controlling the harpooning dynamic of these excited laser-induced intracluster reactions.

It is clear that $a b$ initio calculations of the potential energy surface involving the heavy alkaline-earth atom reactions will provide reliable potential energy surfaces for a complete understanding of many chemical reactions which nowadays can only be covered at a phenomenological level. Indeed this will be particularly interesting and useful for the dynamical and spectroscopic studies of chemical reactions in large clusters or helium droplets. The development of these new theoretical approaches as for example DFT or Monte Carlo Diffusion methods will trigger the investigation of alkaline-earth atom reactions with large organic molecules and bio molecules and it will pave the way to understanding many biological and physiological reactions in which charge transfer processes involving these alkaline-earth atoms seem to play a crucial role.

\section{Acknowledgements}

KCL is grateful for the financial support of the National Science Council, Republic of China, under contract no. NSC94-2113-M-002-013. AGU acknowledges a visiting Professorship from the National Academy of Sciences of Taiwan during which part of this review was prepared. He is also grateful for the current financial support of his research group by the Ministerio de Educación y Ciencia of Spain (grant CTQ2004-3468) and from the Government of the Comunidad de Madrid, the typing of part of the manuscript by Arantxa Vera, and the assistance of Dr Skowronek with figures 3 and 34 .

\section{References}

[1] D. R. Herschbach, Faraday Discussion of the Chemical Society. 55, 223 (1973).

[2] H. W. Cruse, P. D. Dagdigian, and R. N. Zare, in Faraday Discussion of the Chemical Society 55, 277 (1973).

[3] C. T. Rettner and R. N. Zare, J. Chem. Phys. 77, 2416 (1982).

[4] K. C. Lin and R. Vetter, Int. Rev. Phys. Chem. 21, 357 (2002). 
[5] A. González Ureña and R. Vetter, Int. Re. Phys. Chem. 15, 375 (1996).

[6] W. H. Breckenridge and H. Umemoto, J. Chem. Phys. 75, 4153 (1981).

[7] W. H. Breckenridge and H. Umemoto, J. Chem. Phys. 80, 4168 (1984).

[8] W. H. Breckenridge and H. Umemoto, J. Chem. Phys. 81, 3852 (1984).

[9] K. C. Lin and H. C. Chang, J. Chem. Phys. 90, 6151 (1989).

[10] S. Bililign and P. D. Kleiber, J. Chem. Phys. 96, 213 (1992).

[11] S. Bililign and P. D. Kleiber, Phys. Rev. A42, 6938 (1990).

[12] S. Bililign, P. D. Kleiber, W. R. Kearny, and K. M. Sando, J. Chem. Phys. 96, 218 (1992).

[13] X. Huang, J. Zhao, G. Xing, X. Wang, and R. Bersohn, J. Chem. Phys. 104, 1338 (1996).

[14] L. H. Fan, J. J. Chen, Y. Y. Lin, and W. T. Luh, J. Phys. Chem. A 103, 1300 (1999).

[15] P. Hering, P. R. Brooks, R. F. Curl, R. S. Jodson, and F. S. Lowe, Phys. Rev. Lett. 44, 687 (1980).

[16] K. Tsukiyama, M. B. Kata, and R. Bershohn, J. Chem. Phys. 84, 1934 (1980).

[17] N. F. Scherer, L. R. Khundkar, R. B. Bernstein, and A. H. Zewail, J. Chem. Phys. 87, 145 (1987).

[18] W. J. V. D. Zande, R. Zhang, R. N. Zare, K. G. M. Kendrick, and J. J. Valentini, J. Phys. Chem. 95, 8205 (1991).

[19] M. Brouard, S. P. Duxon, P. A. Enriquez, R. Sayos, and J. P. Simons, J. Phys. Chem. 95, 8169 (1991).

[20] M. Brouard, S. P. Duxon, P. A. Enriquez, and J. P. Simons, J. Chem. Phys. 97, 7414 (1992).

[21] Y. L. Chang, L. Chen, M. K. Hsiao, J. J. Chen, and K. C. Lin, J. Chem. Phys. 122, 084315 (2005).

[22] R. L. Farrow and D. W. Chandler, J. Chem. Phys. 89, 1994 (1988).

[23] J. J. Chen and K. C. Lin, J. Chem. Phys. 119, 8785 (2003).

[24] J. J. Chen, M. K. Hsiao, and K. C. Lin, J. Chem. Phys. 123, 121101 (2005).

[25] D. A. V. Kliner, D. E. Adelman, and R. N. Zare, J. Chem. Phys. 95, 1648 (1991).

[26] M. Brouard, I. Burak, S. D. Gatenby, D. Hart, and D. Minayev, J. Chem. Phys. 110, 11335 (1999).

[27] M. Brouard, S. D. Gatenby, D. M. Joseph, and C. Vallance, J. Chem. Phys. 113, 3162 (2000).

[28] D. H. Levy, in Summer School on Reaction Dynamics and Spectroscopy edited by A. Requena, (Universidad de Murcia, Murcia, Spain, 1989).

[29] A. González Ureña, R. B. Bernstein, and G. R. Phillips, J. Chem. Phys. 62, 1818 (1975).

[30] T. E. Gough, M. Mengel, P. A. Rowntree, and G. Scoles, J. Chem. Phys. 83, 4958 (1985).

[31] J. B. Hopkins, P. R. R. Laudridge-Smith, M. D. Morse, and R. E. Smalley, J. Chem. Phys. 78, 1627 (1983).

[32] J. M. Mestdagh, M. A. Gaveau, C. Gee, O. Sublemontier, and J. P. Visticot, Int. Rev. Phys. Chem. 16, 215 (1997).

[33] V. C. Y. Kong, F. R. Foulkes, D. W. Kirk, and J. T. Hinatsu, Int. J. Hydrog. Energy 24, 665 (1999).

[34] R. P. Blickensderfer, K. D. Jordan, N. Adams, and W. H. Breckenridge, J. Chem. Phys. 86, 1930 (1982).

[35] W. H. Breckenridge and J. H. Wang, Chem. Phys. Lett. 137, 195 (1987).

[36] W. H. Breckenridge, J. Phys. Chem. 100, 14840 (1996).

[37] P. D. Kleiber, A. M. Lyyra, K. M. Sando, S. P. Heneghan, and W. C. Stwalley, Phys. Rev. Lett. 54, 2003 (1985).

[38] P. D. Kleiber, A. M. Lyyra, K. M. Sando, S. V. Zafiropulos, and W. C. Stwalley, J. Chem. Phys. 85, 5493 (1986).

[39] P. D. Kleiber, W. C. Stwalley, and K. M. Sando, Annu. Rev. Phys. Chem. 44, 13 (1993).

[40] P. Chaquin, A. Sevin, and H. Yu, J. Phys. Chem. 89, 2813 (1985).

[41] K. C. Lin and C. T. Huang, J. Chem. Phys. 91, 5387 (1989).

[42] D. K. Liu, T. L. Chin, and K. C. Lin, Phys. Rev. A50, 4891 (1994).

[43] Y. R. Ou, D. K. Liu, and K. C. Lin, J. Chem. Phys. 108, 1475 (1998).

[44] R. Schinke, Photodissociation Dynamics: Spectroscopy and Fragmentation of Small Polyatomic Molecules (Cambridge University Press, Cambridge, UK, 1993).

[45] Y. R. Ou, Y. M. Hung, and K. C. Lin, J. Phys. Chem. A 103, 7938 (1999).

[46] Y. M. Hung and K. C. Lin, J. Phys. Chem. A 105, 41 (2001).

[47] D. K. Liu and K. C. Lin, Chem. Phys. Lett. 274, 37 (1997).

[48] D. K. Liu and K. C. Lin, Chem. Phys. Lett. 304, 336 (1999).

[49] D. K. Liu, K. C. Lin, and J. J. Chen, J. Chem. Phys. 113, 5302 (2000).

[50] G. C. Schatz, A. Papaioannou, L. A. Pederson, L. B. Harding, T. Hollebeck, T. S. Ho, and H. Rabitz, J. Chem. Phys. 107, 2340 (1997).

[51] Y. T. Hsu, J. H. Wang, and K. Liu, J. Chem. Phys. 107, 2351 (1997).

[52] K. Mikulecky and K. H. Gericke, J. Chem. Phys. 96, 7490 (1992).

[53] K. Mikulecky and K. H. Gericke, J. Chem. Phys. 98, 1244 (1993).

[54] J. Han, X. Chen, and B. R. Weiner, Chem. Phys. Lett. 332, 243 (2000).

[55] D. Neuhauser, R. S. Judson, D. J. Kouri, D. E. Adelman, N. E. Shafer, D. A. V. Kliner, and R. N. Zare, Science 257, 519 (1992).

[56] K. H. Kim, H. S. Lee, Y. S. Lee, and G. H. Jeung, J. Chem. Phys. 116, 589 (2002).

[57] D. K. Liu, J. J. Chen, C. F. Nien, and K. C. Lin, J. Chem. Phys. 111, 5277 (1999). 
[58] Y. W. Song, J. J. Chen, M. K. Hsiao, K. C. Lin, and Y. M. Hung, J. Chem. Phys. 120, 2774 (2004).

[59] X. Tan, P. J. Dagdigian, and M. H. Alexander, Faraday Discuss. 118, 387 (2001).

[60] J. I. Steinfeld, J. S. Francisco, and W. L. Has, Chemical Kinetics and Dynamics, 2nd ed. (Prentice-Hall, London, 1999).

[61] W. H. Breckenridge and H. Umemoto, J. Chem. Phys. 75, 698 (1981).

[62] W. H. Breckenridge and H. Umemoto, J. Chem. Phys. 77, 4464 (1982).

[63] P. Chaquin, A. Papakondylis, C. Giessner-Prettre, and A. Sevin, J. Phys. Chem. 94, 7352 (1990).

[64] P. D. Kleiber, in Kinetics and Dynamics of Reactions of Small Radicals, edited by A. Wagner and K. Liu (World Scientific, Singapore, 1995).

[65] D. K. Liu, Y. R. Ou, and K. C. Lin, J. Chem. Phys. 104, 1370 (1996).

[66] T. H. Wong and P. D. Kleiber, J. Chem. Phys. 102, 6476 (1995).

[67] T. H. Wong, C. Freel, P. D. Kleiber, and K. M. Sando, J. Chem. Phys. 108, 5723 (1998).

[68] C. F. Nien and K. C. Lin, J. Chem. Phys. 109, 7821 (1998).

[69] J. J. Chen, Y. W. Song, K. C. Lin, and Y. H. Hung, J. Chem. Phys. 118, 4938 (2003).

[70] L. Chen, M. K. Hsiao, Y. L. Chang, and K. C. Lin, J. Chem. Phys. 124, 024304 (2006).

[71] R. Rubino, J. M. Williamson, and T. A. Miller, J. Chem. Phys. 103, 5964 (1995).

[72] J. G. McCaffrey, J. M. Parnis, G. A. Ozin, and W. H. Breckenridge, J. Phys. Chem. 89, 4945 (1985).

[73] J. G. McCaffrey, J. M. Parnis, and G. A. Ozin, J. Chem. Phys. 89, 1858 (1988).

[74] J. G. McCaffrey and G. A. Ozin, J. Chem. Phys. 89, 1839 (1988).

[75] D. J. Bogan and D. W. Setser, J. Chem. Phys. 64, 586 (1976).

[76] H. Umemoto, T. Sato, K. Takano, S. Tsunashima, K. Kuwahara, K. Sato, H. Ikeda, F. Misaizu, and K. Fuke, Chem. Phys. Lett. 214, 271 (1993).

[77] H. Umemoto, S. Tsunashima, H. Ikeda, K. Takano, K. Kuwahara, K. Sato, K. Yokoyama, F. Misaizu, and K. Fuke, J. Chem. Phys. 101, 4803 (1994).

[78] S. Castillo, A. Ramirez-Solis, D. Diaz, E. Poulain, and O. Novaro, Mol. Phys. 81, 825 (1994).

[79] S. Castillo, E. Poulain, and O. Novaro, Int. J. Quant. Chem. 25, 577 (1991).

[80] A. C. Luntz, J. Chem. Phys. 73, 1143 (1980).

[81] P. M. Aker, J. J. A. Obrien, and J. J. Sloan, J. Chem. Phys. 84, 745 (1986).

[82] C. R. Park and J. Wiesenfeld, J. Chem. Phys. 95, 8166 (1991).

[83] H. Umemoto, T. Nakae, H. Hashimoto, K. Kongo, and M. Kawasaki, J. Chem. Phys. 109, 5844 (1998).

[84] C. R. Brazier and P. F. Bernath, J. Chem. Phys. 91, 4548 (1989).

[85] B. Bourguignon, M. A. Gargoura, J. Rostas, and G. Taieb, J. Phys. Chem. 91, 2080 (1987).

[86] J. M. Teule, J. Mes, J. Bulthuis, M. H. M. Jansem, and S. Stolte, J. Phys. Chem. A 102, 9482 (1998).

[87] J. M. Teule, M. H. M. Jansem, and S. Stolte, J. Chem. Phys. 110, 10792 (1999).

[88] M. Esteban, M. Garay, J. M. Garcia-Tejero, E. Verdasco, and A. González Ureña, Chem. Phys. Lett. 230, 525 (1994).

[89] F. Engelke and K. H. Meiwes-Broer, in Selectivity in Chemical Reactions, edited by J. C. Whitehead (Kluver Dordrecht, 1988), Vol. 245, pp 135 - 146, NATO ASI series.

[90] K. Johnson, R. Pease, and J. P. Simons, Molec. Phys. 4, 955 (1984).

[91] M. G. Prisant, C. T. Retner, and R. N. Zare, J. Chem. Phys. 75, 2222 (1981).

[92] W. S. Hartree, J. P. Simons, and A. González Ureña, J. Chem. Soc. Faraday Trans. 86, 17 (1990).

[93] M. Garay, M. Esteban, E. Verdasco, and A. González Ureña, Chem. Phys. 195, 235 (1995).

[94] R. J. Li, K. I. Han, F. E. Li, R. C. Lu, G. Z. He, and N. Q. Lou, Chem. Phys. Lett. 220, 281 (1994).

[95] M. Menéndez, M. Garay, E. Verdasco, and A. González Ureña, J. Chem. Phys. 99, 2760 (1993).

[96] M. L. Wang, K. L. Han, J. P. Zhan, V. W. K. Wu, G. Z. He, and N. Q. Lou, Chem. Phys. Lett. 278, 307 (1997).

[97] J. P. Zhan, H. P. Yang, K. L. Han, M. L. Wang, W. Q. Deng, G. Z. He, and N. Q. Lou, J. Chem. Phys. 109, 1819 (1998).

[98] J. P. Zhan, H. P. Yang, K.H Han, M. L. Wang, W. Q. Deng, G. Z. He, and N. Q. Lou, Chem. Phys. 109, 1819 (1998).

[99] J. P. Zhan, H. P. Yang, K. L. Han, W. Q. Deng, G. Z. He, and N. Q. Lou, J. Phys. Chem. A 101, 7548 (1997).

[100] A. González Ureña, Ad. Chem. Phys. 66, 213 (1987).

[101] E. Verdasco, V. Sáez Rabanos, and A. González Ureña, Laser Chem. 10, 51 (1989).

[102] C. Naulin and M. Costes, Chem. Phys. Lett. 310, 231 (1999).

[103] B. G. Wicke, J. Chem. Phys. 78, 6036 (1983).

[104] M. Garay, J. M. Orea, A. González Ureña, and G. Roberts, Mol. Phys. 97, 967 (1999).

[105] M. Garay, J. M. Orea, and A. González Ureña, Chem. Phys. 216, 365 (1997).

[106] C. Vítores, M. R. Candori, F. Pirani, and V. Aquilanti, J. Chem. Phys. 112, 770 (2000).

[107] C. A. Rinaldi, M. De Castro, J. Cáceres, and A. González Ureña, Recent. Res. Develop. Chem. Phys. 3, 1 (2002).

[108] K. S. Kalogerakis and R. N. Zare, J. Chem. Phys. 104, 7947 (1996). 
[109] H. F. Davis, A. G. Suits, H. Hou, Y. T. Lee, and B. Bunsenges, Phys. Chem. 94, 1193 (1990).

[110] M. Menzinger, in Selectivity in Chemical Reactions, edited by J. C. Whithehead (Kluver, Academic Dordrecht, 1988).

[111] A. G. Suits, H. Hongtao, H. F. Davis, and Y. T. Lee, J. Chem. Phys. 95, 8178 (1991).

[112] M. Menzinger, in Gas Phase Chemiluminescence and Chemi-ionization, edited by A. Foutjin (Elsevier, Amsterdam, 1985), pp. 25-66.

[113] E. E. B. Campbell, H. Schmidt, and I. V. Hertel, Adv. Chem. Phys. 72, 37 (1998).

[114] S. Stolte, in Atomic and Molecular Beam Methods, edited by G. Scoles (Oxford University Press, 1998) Vol. 1, pp. 631-652.

[115] M. H. M. Janssen, D. H. Parker, and S. Stolte, J. Phys. Chem. 95, 8142 (1991).

[116] M. H. M. Janssen, D. H. Parker, and S. Stolte, J. Phys. Chem. 100, 16066 (1984).

[117] A. J. Meijer, H. M. Groenenboom, and V. D. Avoird, J. Phys. Chem. 100, 16072 (1996).

[118] M. Menzinger, Polon. Phys. Acta. A73, 85 (1988).

[119] S. M. Lin, C. A. Mins, and R. R. Herm, J. Phys. Chem. 77, 569 (1973).

[120] C. A. Mims, S. H. Lin, and R. R. Herm, J. Chem. Phys. 57, 3099 (1972).

[121] K. L. Han, X. G. Zheng, B. F. Sun, and G. Z. He, Chem. Phys. Lett. 181, 474 (1991).

[122] B. Pranszke, P. Kierkowski, and A. Kowalski, Chem. Phys. Lett. 317, 220 (2000).

[123] F. Beitia, F. Castaño, and M. N. R. Sánchez, J. Photochem. Photobiol. A: Chem. 62, 1 (1991).

[124] B. Soep, C. J. Whithani, A. Keller, and J. P. Visticot, Faraday Discuss. Chem. Soc. 91, 191 (1991)

[125] J. C. Polanyi and J. X. Wang, J. Phys. Chem. 99, 13691 (1995).

[126] K. Lin, J. C. Polanyi, and S. Yang, J. Chem. Phys. 98, 5431 (1993).

[127] C. A. Rinaldi, K. Gasmi, S. Skowronek, and A. González Ureña, J. Chem. Phys. 124, 244309 (2006).

[128] S. Skowronek, R. Pereira, and A. González Ureña, J. Phys. Chem. A 101, 7468 (1997).

[129] S. Skowronek and A. González Ureña, Prog. Reaction Kinetics and Mechanisms 24, 101 (1999).

[130] V. Stert, H. H. Ritze, P. Farmanara, and W. Radloff, Phys. Chem. Chem. Phys. 3, 3939 (2001).

[131] V. Stert, P. Farmanara, H. H. Ritze, W. Radloff, K. Gasmi, and A. González Ureña, Chem. Phys. Lett. 337, 299 (2001).

[132] J. M. Mestdagh, F. Spiegelman, E. Gloaguen, M. Collier, F. Lepetit, M. A. Gavean, C. Sanz Sanz, and B. Soep, J. Phys. Chem. A 110, 7355 (2006).

[133] P. Farmanara, V. Stert, W. Radloff, S. Skowronek, and A. González Ureña, Chem. Phys. Lett. 304, 127 (1999).

[134] C. A. Rinaldi, C. M. Santiveri, G. Tardajos, and A. González Ureña, Chem. Phys. Lett. 274, 29 (1997).

[135] K. Gasmi, S. Skowronek, and A. González Ureña, Eur. Phys. J. D 33, 399 (2005). 
Copyright of International Reviews in Physical Chemistry is the property of Taylor \& Francis Ltd and its content may not be copied or emailed to multiple sites or posted to a listserv without the copyright holder's express written permission. However, users may print, download, or email articles for individual use. 